Provided for non-commercial research and education use. Not for reproduction, distribution or commercial use.

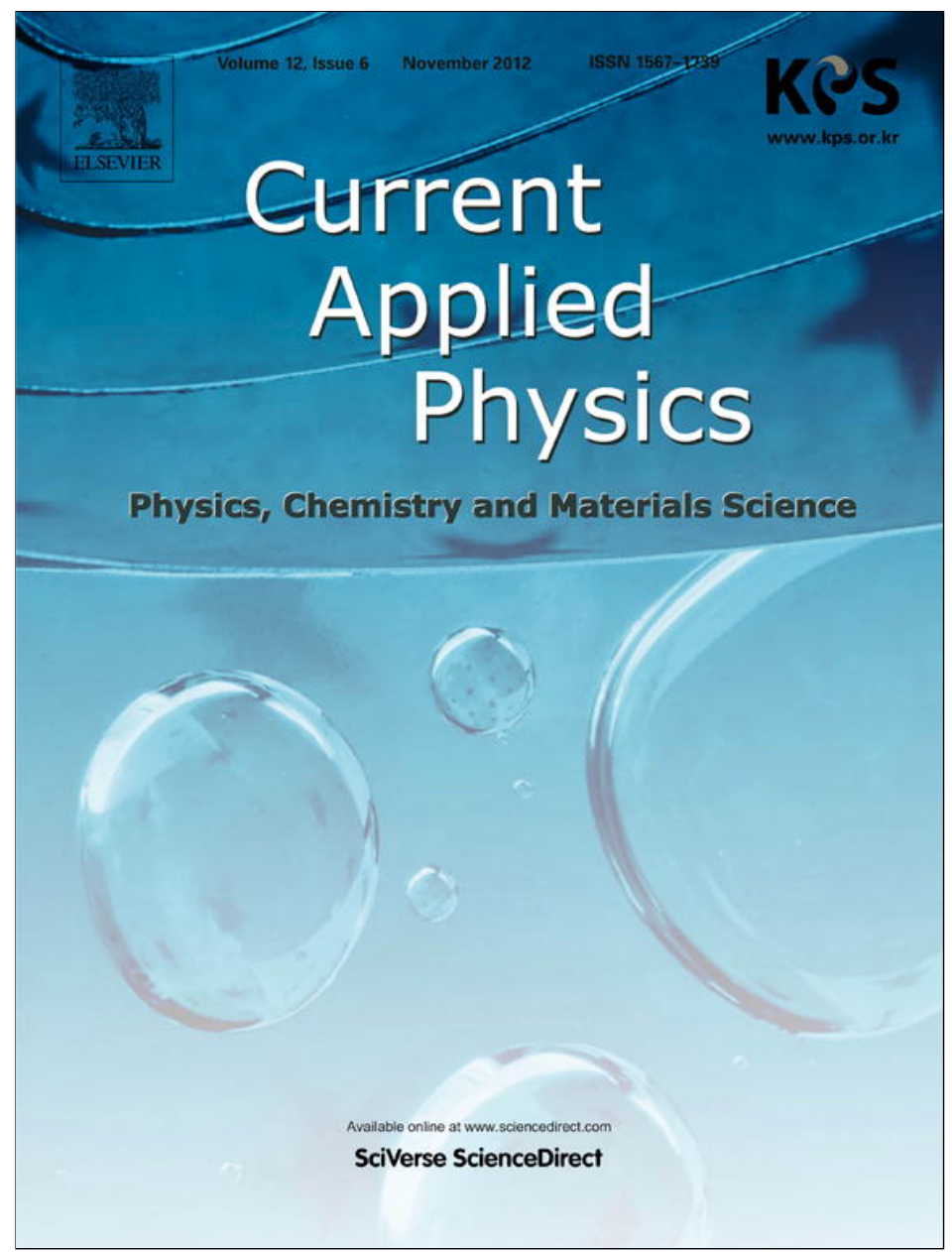

This article appeared in a journal published by Elsevier. The attached copy is furnished to the author for internal non-commercial research and education use, including for instruction at the authors institution and sharing with colleagues.

Other uses, including reproduction and distribution, or selling or licensing copies, or posting to personal, institutional or third party websites are prohibited.

In most cases authors are permitted to post their version of the article (e.g. in Word or Tex form) to their personal website or institutional repository. Authors requiring further information regarding Elsevier's archiving and manuscript policies are encouraged to visit:

http://www.elsevier.com/copyright 
Review

\title{
A new era for liquid crystal research: Applications of liquid crystals in soft matter nano-, bio- and microtechnology
}

\author{
Jan P.F. Lagerwall ${ }^{\mathrm{a}, \mathrm{b}, *}$, Giusy Scalia ${ }^{\mathrm{a}, \mathrm{b}, *}$ \\ ${ }^{a}$ Seoul National University, Graduate School of Convergence Science E Technology, 864-1 Iui-dong, Yeongtong-gu, Suwon-si, Gyeonggi-do 443-270, Republic of Korea \\ ${ }^{\mathrm{b}}$ Advanced Institutes of Convergence Technologies, Department of Nanoscience E' Technology, 864-1 Iui-dong, Yeongtong-gu, Suwon-si, Gyeonggi-do 443-270, Republic of Korea
}

\section{A R T I C L E I N F O}

\section{Article history:}

Received 14 February 2012

Accepted 13 March 2012

Available online 30 March 2012

\section{Keywords:}

Liquid crystal

Lyotropic

Thermotropic

Nanotechnology

Soft matter

Composites

Colloids

Biomembranes

\begin{abstract}
A B S T R A C T
Liquid crystals constitute a fascinating class of soft condensed matter characterized by the counterintuitive combination of fluidity and long-range order. Today they are best known for their exceptionally successful application in flat panel displays, but they actually exhibit a plethora of unique and attractive properties that offer tremendous potential for fundamental science as well as innovative applications well beyond the realm of displays. Today this full breadth of the liquid crystalline state of matter is becoming increasingly recognized and numerous new and exciting lines of research are being opened up. We review this exciting development, focusing primarily on the physics aspects of the new research thrusts, in which liquid crystals - thermotropic as well as lyotropic - often meet other types of soft matter, such as polymers and colloidal nano- or microparticle dispersions. Because the field is of large interest also for researchers without a liquid crystal background we begin with a concise introduction to the liquid crystalline state of matter and the key concepts of the research field. We then discuss a selection of promising new directions, starting with liquid crystals for organic electronics, followed by nanotemplating and nanoparticle organization using liquid crystals, liquid crystal colloids (where the liquid crystal can constitute either the continuous phase or the disperse phase, as droplets or shells) and their potential in e.g. photonics and metamaterials, liquid crystal-functionalized polymer fibers, liquid crystal elastomer actuators, ending with a brief overview of activities focusing on liquid crystals in biology, food science and pharmacology.
\end{abstract}

๑) 2012 Elsevier B.V. All rights reserved.

\section{Contents}

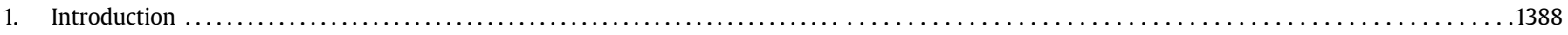

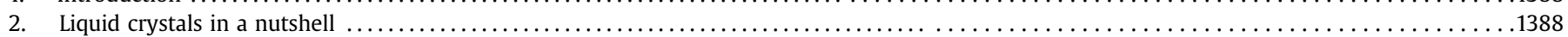

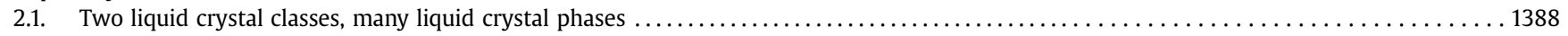

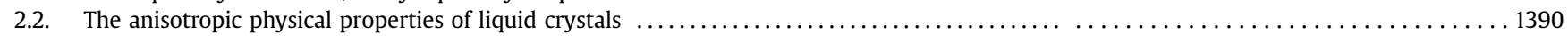

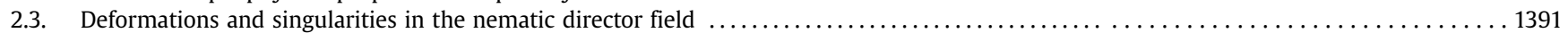

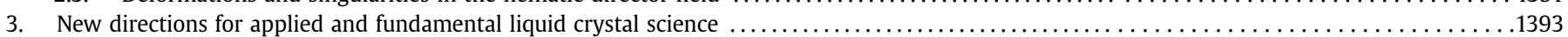

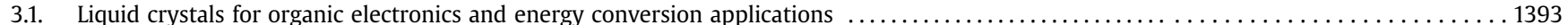

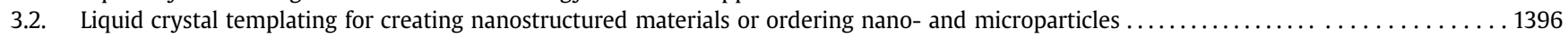

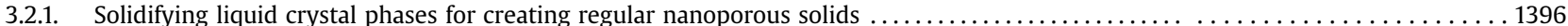

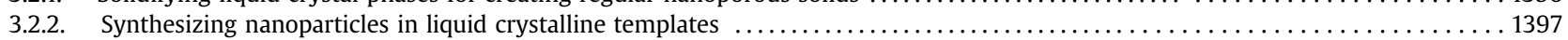

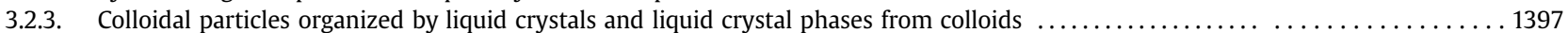

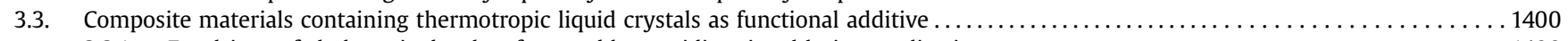

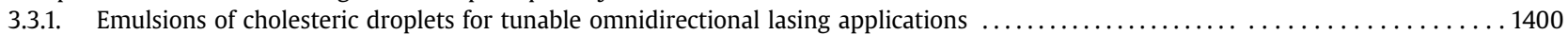

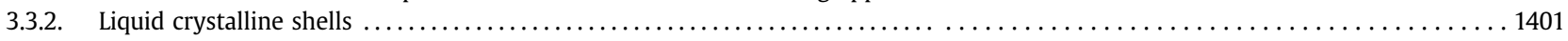

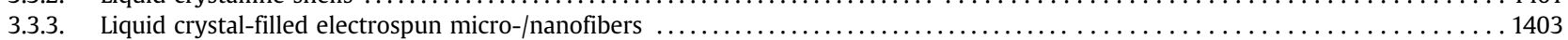

\footnotetext{
* Corresponding authors. Seoul National University, Graduate School of Convergence Science \& Technology, 864-1 Iui-dong, Yeongtong-gu, Suwon-si, Gyeonggi-do 443-270, Republic of Korea.

E-mail addresses: jan.lagerwall@lcsoftmatter.com (J.P.F. Lagerwall), giusy.scalia@solcanta.com (G. Scalia).

URL: http://www.snm.lcsoftmatter.com, http://www.solcanta.com
} 


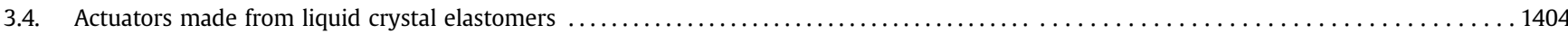

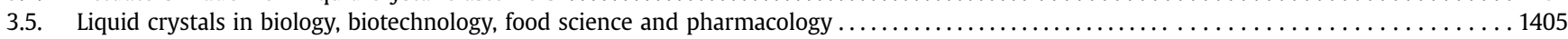

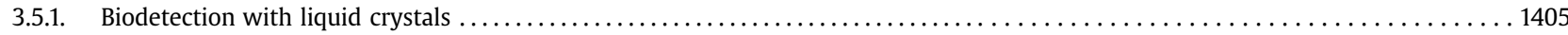

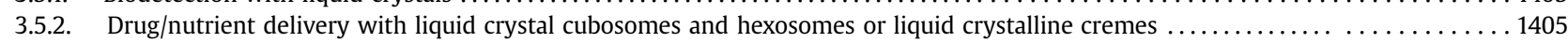

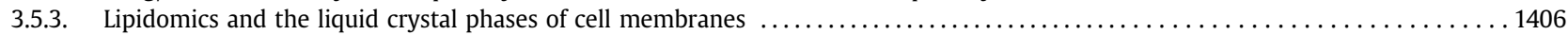

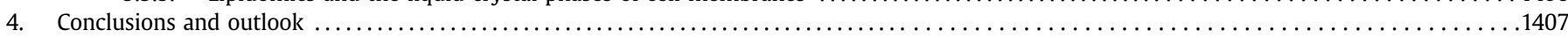

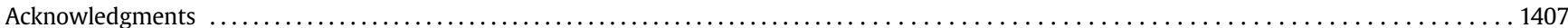

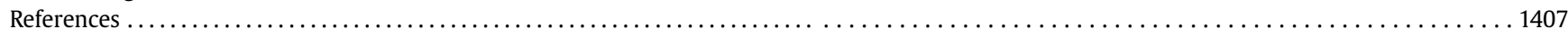

\section{Introduction}

It is almost 125 years since the Prague scientist Friedrich Reinitzer in 1888 observed a curious behavior with double melting points of cholesterol benzoate, a discovery that today is widely recognized as the birth of liquid crystal science. The discovery and the new area of research that it triggered, engaging physicists and chemists around the world, remained of almost pure academic interest for some 75 years, until the idea occurred to utilize the simplest liquid crystal phase - the nematic - in display devices [1]. The impact of this idea was hardly clear at the time, but today we can look back at the development of liquid crystal displays (LCDs) and conclude that this application of the curious materials that liquid crystals constitute have entirely revolutionized our society. The explosive progress in display design and construction have made old-style cathode ray tube displays dinosaurs, and it has made possible a number of entirely new devices such as smart phones, laptop and tablet computers, digital projectors and dynamic information billboards.

When the promise of liquid crystal displays became clear in the 1970s this stimulated great activity among academic and industrial researchers alike, and for quite a long time this single application strongly dominated the international research efforts in the field. As a result of this long engagement, stimulated by the commercial success, liquid crystal display technology is today so advanced that the continued research and development is conducted primarily in industry labs. Academic liquid crystal science has, in contrast, during the last few years shown a clear trend of moving away from display research and instead focusing on topics that can be very different and in many respects even more stimulating [2], for instance in new uses in optics, nano-/micro manipulation, novel composites and biotechnology. The academic research field is presently experiencing a very creative and explorative phase, giving the field a rebirth into a metamorphosed state in which other unique aspects of liquid crystals are brought forward and where the curious liquid crystalline states of matter are studied in new contexts.

In their encounter with other very relevant fields of soft matter science, most conspicuously the science of polymers, colloids, nanotechnology and biotechnology, highly interesting and attractive research fields are emerging, many with considerable potential for applications of entirely new kinds. The unique subtle balance between order and fluidity that characterizes liquid crystals is not only deeply fascinating from a scientific point of view, but it gives rise to a broad range of spectacular phenomena that are far from fully explored. It is the aim of this review article to give a snapshot of the exciting development that the field is currently experiencing, with an emphasis on the physics and materials science issues involved. The recent developments of liquid crystal chemistry (which are also very impressive and innovative) have been reviewed elsewhere, see e.g. [3-12]. We have tried to give a bird's eye view of the most exciting current activities in liquid crystalrelated nano-, micro- and biotechnology not related to displays or similar electrooptic devices, providing also a critical analysis of the key outstanding challenges in each field. Since the topics covered are highly interdisciplinary and thus of interest also to readers outside the liquid crystal community we begin with a very condensed introduction to liquid crystals with explanations of the key concepts (an extended version can be found in one of the authors' habilitation thesis [13]), allowing also non-liquid crystal experts to enjoy the science described in the following chapters.

\section{Liquid crystals in a nutshell}

\subsection{Two liquid crystal classes, many liquid crystal phases}

What makes liquid crystals unique is that they are fluids, yet they exhibit long-range order: either the orientation or position, or both, of the phase building blocks are correlated over a long distance. In the nematic phase (abbreviated $\mathrm{N}$ ) the long-range order is of purely orientational type: the anisometric building blocks (often rods or discs) are roughly oriented along one and the same direction, referred to as the director (abbreviated $\mathbf{n}$ ), as illustrated in Fig. 1. In the absence of aligning forces the director varies smoothly throughout a nematic sample without discrete changes (except in defects), hence one often speaks of the director field $\mathbf{n}(\mathbf{r})$, where $\mathbf{r}$ is the space coordinate.

Two main classes of liquid crystal exist. Thermotropic liquid crystals are built up by individual molecules and no further

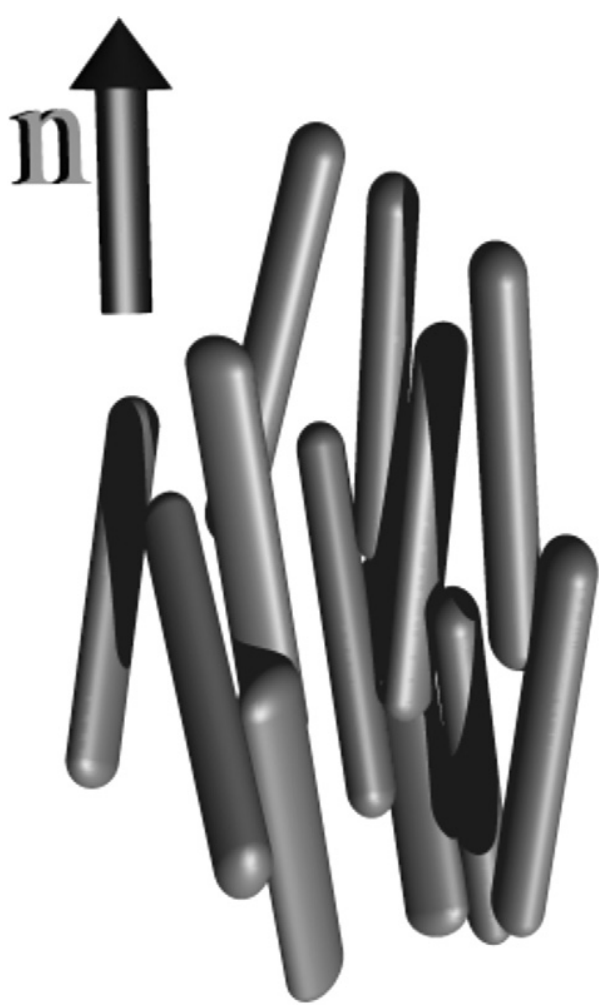

Fig. 1. Cartoon of a nematic phase built from rod-shaped building blocks, drawn with a vertical director $\mathbf{n}$. From [13]. 
molecular species (specifically solvent molecules) are required for the liquid crystal phase formation. Temperature is the fundamental thermodynamic control parameter determining the phase, the two key temperature values being those defining the beginning and end of liquid crystalline order, the melting point $T_{m}$ from the crystalline solid and the clearing point $T_{c}$ into an isotropic liquid. The clearing point owes its name to the fact that a bulk liquid crystal scatters light, whereas the isotropic liquid is clear. Because of their intermediate state between solids and liquids, liquid crystals are sometimes referred to as mesomorphic systems and molecules or other entitites (nanoparticles, molecule aggregates, ...) capable of forming liquid crystal phases are called mesogens or said to be mesogenic. In addition to anisometry, mesogenicity among thermotropic molecules has its origin in a combination of a stiff core (often a linear or circular arrangement of aromatic rings) and flexible terminal chains, generally aliphatic hydrocarbons, cf. the examples in Fig. 2.

Lyotropic liquid crystals form only upon addition of a solvent, most often water. Moreover, the building block of a lyotropic phase is often not one but many molecules (typically on the order of 100), organized into an aggregate called a micelle. The micelle formation is a result of the amphiphilic character of the constituent molecules, generally surfactants, cf. the an-, cat- and nonionic examples in Fig. $2 \mathrm{~d}-\mathrm{f}$, respectively. Micelles, in which the amphiphiles are organized with the non-polar tails inwards, thus protected from water contact by the outwards-directed polar head groups, start forming beyond a limiting amphiphile concentration called the Critical Micelle Concentration, abbreviated CMC. Typical CMC values lie in the $1-10 \mathrm{mM}$ range (about $0.01-0.1 \mathrm{wt} . \%$ ). The CMC does not constitute the onset of liquid crystalline order: the micellar phase in the vicinity of the CMC and quite a substantial regime beyond is isotropic in all properties. Another very important example of amphiphiles forming liquid crystals, although generally not micelles, is given by the amphiphilic lipids (e.g. phospho-, glycoand sphingolipids) that build up the membranes of the cells in our own bodies. The prototypic phospholipid DMPC is shown as example in Fig. 2g. Also amphiphlic block co-polymers can form liquid crystal phases.

Lyotropic liquid crystals can form also by anisometric nonamphiphilic macromolecules or particles such as viruses or inorganic rods or discs in colloidal suspension. This subclass of lyotropics is attracting increasing interest today [14-21]. Polymers that form liquid crystals of this type may be synthetic such as carbon nanotubes or the polyaramide chains that are processed via a liquid crystalline state into Kevlar $^{\circledR}$ fibers, or they may be biological, e.g. DNA.

The nematic is the simplest liquid crystal phase, exhibiting longrange orientational but no positional order. If we add 1D positional order, i.e. a periodic distribution of the molecules along one specific direction, we get the so-called smectic (thermotropic) or lamellar (lyotropic) phases, where the molecules (typically rod-shaped) are organized into layers. In smectics the degree of positional order is still not very high and molecules frequently move from one layer to the next, but in the lamellar phases the amphiphiles build up bilayers that are separated by an aqueous region that can be much thicker than the bilayer, making the layering much more distinct. Within the layers the molecules may be on the average perpendicular to the layers (we call this a smectic-A, or SmA, state for thermotropics, and an $\mathrm{L}_{\alpha}$ state for lamellar lyotropics) or they can be inclined with a certain angle (thermotropic SmC or lyotropic $\mathrm{L}_{\beta}^{\prime}$; the difference between $\mathrm{L}_{\alpha}$ and $\mathrm{L}_{\beta}^{\prime}$ however goes far beyond the issue of molecule tilt [13]).

The next step in long-range positional order in liquid crystal phases is encountered among the columnar phases, where the a

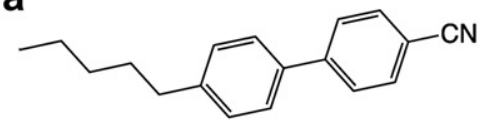

5CB (4-Cyano-4'-pentylbiphenyl):

Cr. $23 \mathrm{~N} 35$ Iso. Iso. $/{ }^{\circ} \mathrm{C}$

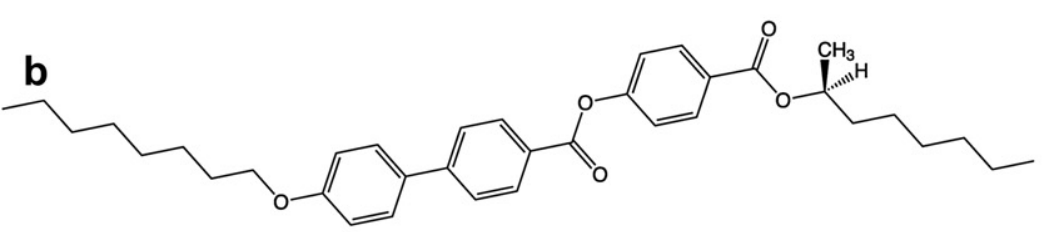

(S)-MHPOBC [(S)-4'-Octyloxy-biphenyl-4-carboxylic acid 4-(I-methyl-heptyloxycarbonyl)-phenyl ester]:

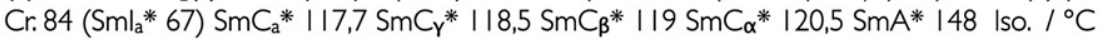
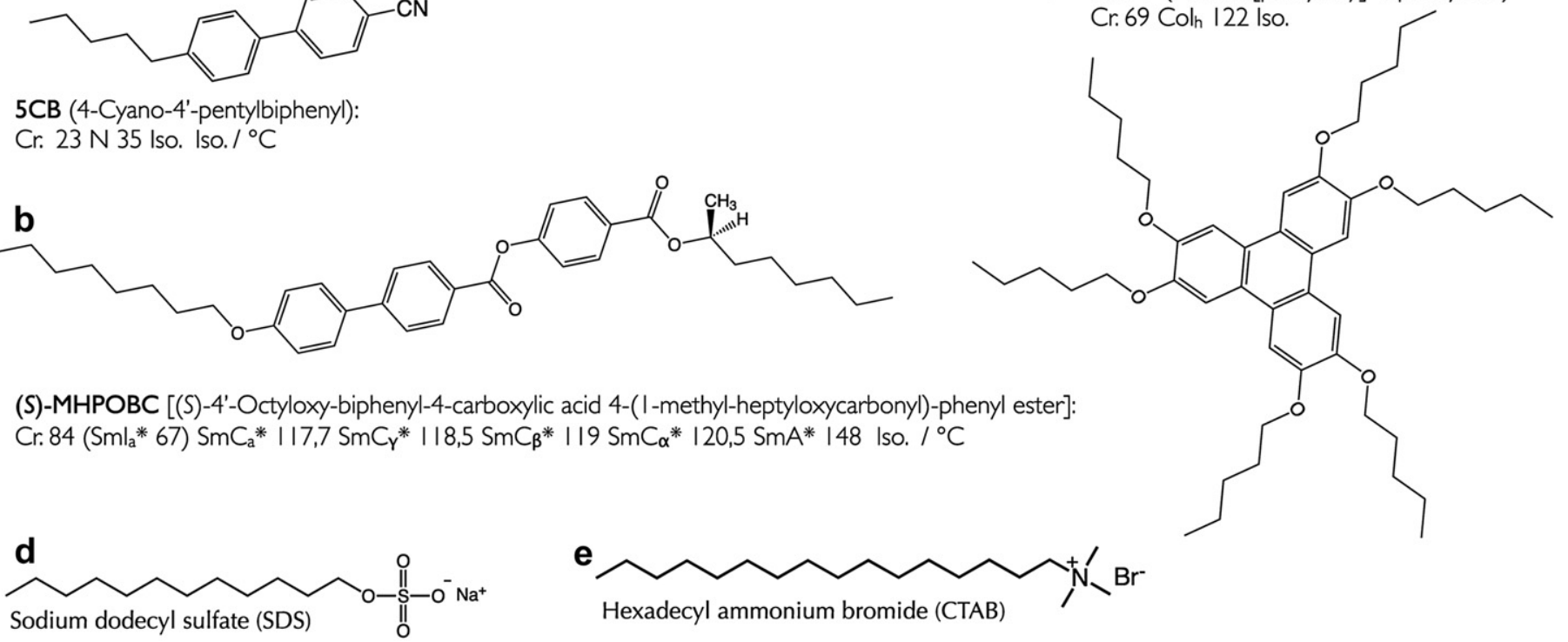

Sodium dodecyl sulfate (SDS)
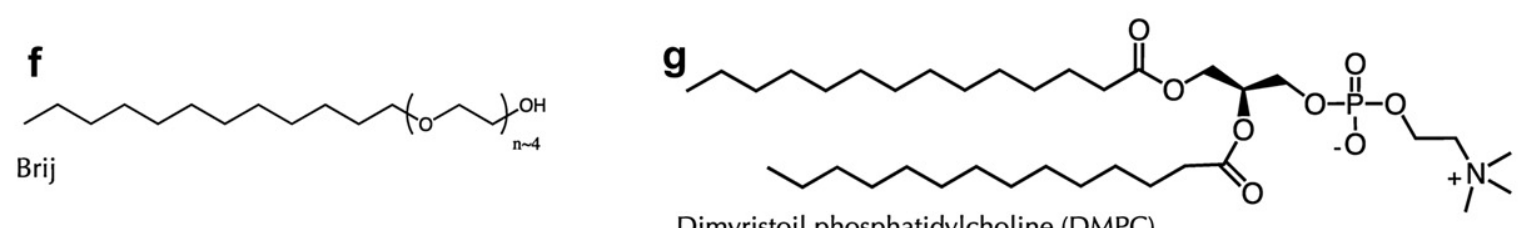

Dimyristoil phosphatidylcholine (DMPC)

Fig. 2. Some typical rod-shaped thermotropic mesogens (a-b), a classic discotic thermotropic mesogen (c) and four commonly occurring amphiphiles (d-g). 

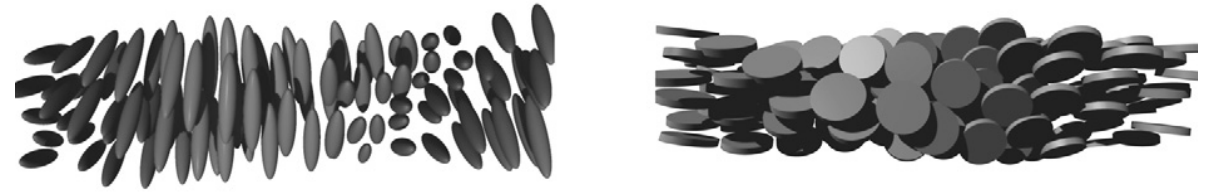

Fig. 3. Cartoons of helical director modulation in the chiral nematic (cholesteric) phase with rod- and disc-shaped building blocks, respectively. Each rod or disc represents either a mesogen (thermotropics) or micelle (lyotropics) with the corresponding shape. Note that, for clarity, the pitch has been drawn orders of magnitude smaller than in reality and the local degree of order is much too high. From [13].

building blocks are positioned on a 2D lattice, but without longrange positional correlation in the third direction. In the thermotropic case the columnar order is typically formed by discotic mesogens, the aromatic cores stacking up as in piles of coins, each pile being located on a point of a lattice that is often hexagonal. In the lyotropic columnar phases we have 'infinitely' long rod-shaped micelles positionally correlated on a 2D lattice in a plane perpendicular to the rods.

If we add chiral molecules to a liquid crystal phase, either as non-mesogenic dopants or by using chiral mesogens, the macroscopic liquid crystal phase is generally also chiral. The presence of stereoisomerically enriched chiral molecules is for thermotropic liquid crystals generally indicated by adding a star to the shorthand of the corresponding achiral phase, e.g. $\mathrm{N}^{*}$ for a chiral nematic. In lyotropics the same indication is used for chiral nematics but the convention has not been adopted for lamellar and columnar phases.

The best studied chiral liquid crystal is the chiral nematic phase, often referred to as the cholesteric phase. The chirality here expresses itself as a helical modulation of the director along an axis perpendicular to $\mathbf{n}$, cf. Fig. 3. In smectic phases, a helical director modulation perpendicular to $\mathbf{n}$ would break the layers and is thus not normally admitted (an exception is the rare class of twist-grain boundary phases). The $\mathrm{SmA}^{*}$ phase therefore has the same structure as an achiral SmA phase, but its physical properties are quite different from its achiral analog [22]. In contrast to SmA*, the chiral SmC* phase does allow a helical superstructure, but it applies to the direction along which the molecules tilt away from the layer normal. In other words, $\mathbf{n}$ in SmC* does not twist as in $\mathrm{N}^{*}$, but the tilting direction precesses helically along the smectic layer normal. The SmC* phase is different from its non-chiral analog, as well as from any nematic phase, also in that it exhibits a spontaneous polarization and can thus show ferroelectric switching properties [22].

The periodicity of the helix (the pitch $p$ ) can be anywhere from a few hundred nanometers to infinity, for cholesterics as well as $\mathrm{SmC}^{*}$-type phases. If it is short the physical properties can change dramatically compared to the non-chiral phases [23]. The mechanical properties of a short-pitch $\mathrm{N}^{*}$ phase along the helix can start resembling those of a weak solid and for $p$ on the order of visible light wavelengths the periodicity leads to visible Bragg scattering, from $\mathrm{N}^{*}$ as well as from $\mathrm{SmC}^{*}$, referred to as selective reflection. These chiral liquid crystals then become self-assembled photonic crystals with a band gap that can be tuned fairly easily, e.g. by temperature variation.

\subsection{The anisotropic physical properties of liquid crystals}

The macroscopic anisotropy of liquid crystals, a property shared with no other fluid, is the basis for their successful commercial exploitation. The anisometry of the building blocks together with the long-range orientational order generally render liquid crystals optically anisotropic, i.e. their refractive index $n_{\|}$parallel to $\mathbf{n}$ is different from that perpendicular to the director, $n_{\perp}$. For a nonchiral uniaxial material, these two indices are the extreme refractive index values and the director is equivalent to the optic axis of the liquid crystal (please refer to more specialized texts, e.g. [23], for the special case of chiral liquid crystals with short-pitch director modulation). The birefringence of the material is then $\Delta n=n_{\|}-n_{\perp}$. Consequently, if $n_{\|}$is the maximum refractive index, $\Delta n>0$ and we call the liquid crystal positive uniaxial (this is the general case for rod-shaped mesogens), whereas if it is the minimum the phase is negative uniaxial (this is the standard case for discotics).

Since the effect on polarized light of a birefringent material depends critically on the orientation of the optic axis it is important to specify how a liquid crystal is aligned with respect to the direction of light propagation. A device in which the liquid crystal is used for its anisotropic optical properties, e.g. a display, is generally a thin flat film, referred to as a 'cell' in the general case. In the standard configuration light enters perpendicular to the cell plane. When $\mathbf{n}$ is aligned perpendicular to the cell the light ray thus traverses the cell along the optic axis, leading to no effective birefringence (any polarization of the light experiences the ordinary refractive index) and this director alignment is therefore referred to as homeotropic, a term that can be understood as meaning like isotropic. If $\mathbf{n}$ is instead perpendicular to the cell plane we call this planar geometry. At normal incidence to the cell, light will now
Homeotropic

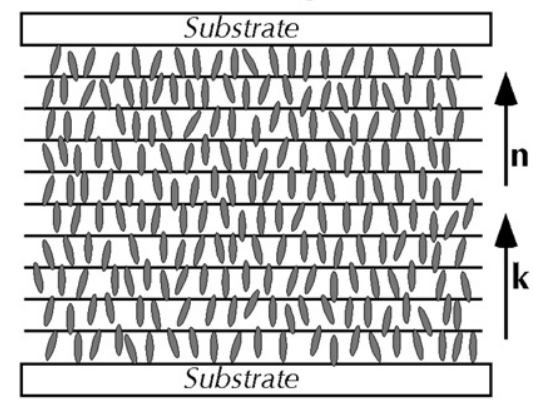

Planar

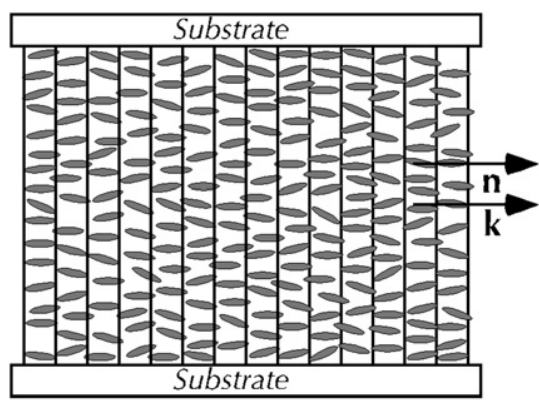

Fig. 4. Schematic drawing of homeotropic and planar alignment, respectively, here illustrated for the case of an SmA phase (layer normal $\mathbf{k}$ parallel to director $\mathbf{n}$ ) confined between parallel flat substrates. Normal light incidence means that the light beam is propagating vertically in the picture. Note that the drawing is not to scale: a smectic layer is some three orders of magnitude thinner than the distance between the sample substrates. From [13]. 


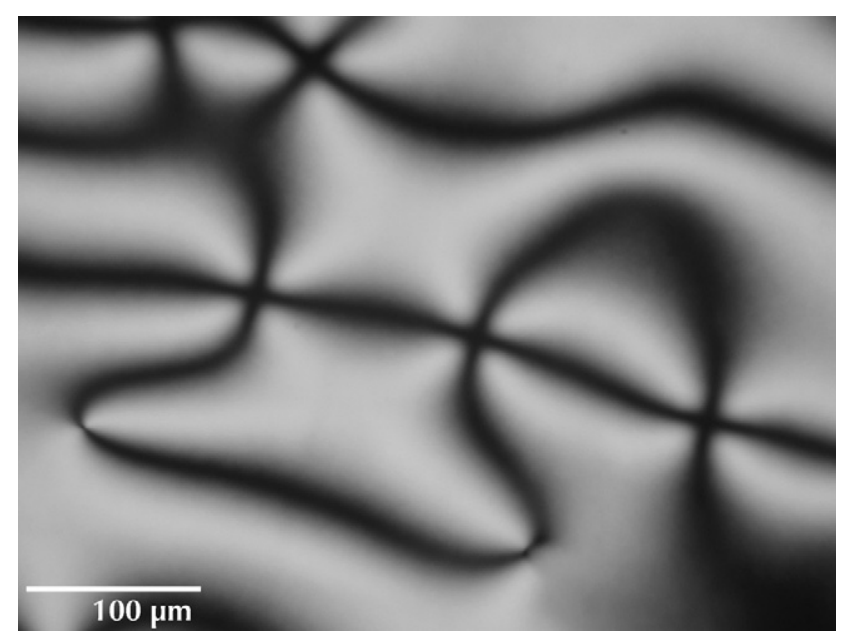

Fig. 5. An example of the characteristic schlieren texture of nematic liquid crystals in degenerate planar alignment. The micrograph is of a lyotropic nematic formed by discshaped micelles, but the same type of texture can be formed by any type of achiral nematic in degenerate planar geometry. From [13]

experience the maximum birefringence. For curved samples, e.g. liquid crystal droplets or shells, the terms homeotropic and planar are still used, then referring to the director alignment with respect to the interface with the continuous phase, although the optic effects are more complex due to the curvature. In Fig. 4 the two principal alignment types are illustrated for the case of a smectic-A phase between flat substrates.

In a planar-aligned nematic sample where $\mathbf{n}(\mathbf{r})$ has no restriction to any particular direction in the sample plane (we refer to this geometry as degenerate planar alignment), the director and thus the optic axis vary randomly throughout the sample area. When observing the sample between crossed polarizers, all locations where $\mathbf{n}$ is parallel to one of the polarizers will appear black since then either the ordinary or the extraordinary component vanishes. Since these locations are continuously connected, forming randomly curving 'threads' or 'streaks', we get a texture that is very characteristic for the nematic phase, cf. the example in Fig. 5. This texture is called a schlieren texture after the German word for streak; Schliere.

The ability to modulate a liquid crystal display element between dark and bright is normally due to the dielectric anisotropy $\Delta \varepsilon=\varepsilon_{\|}-\varepsilon_{\perp}$, with $\varepsilon_{\|}$and $\varepsilon_{\perp}$ being the permittivities along and perpendicular to $\mathbf{n}$, respectively. By applying an electric field this gives us a handle for switching the director orientation between different defined geometries, giving different transmission. When an electric field $\mathbf{E}$ is applied over a dielectric medium such as a liquid crystal, the field will polarize the medium, i.e. an electric dipole moment $\mathbf{P}$ will be induced, proportional to the field strength and to the permittivity of the medium. Since a liquid crystal is dielectrically anisotropic the polarization and the field will be directed differently for any director orientation that is not along $\mathbf{E}$. The result is a dielectric torque $\boldsymbol{\Gamma}_{\varepsilon}=\mathbf{P} \times \mathbf{E}$ on the director, acting to align $\mathbf{n}$ along $(\Delta \varepsilon>0)$ or perpendicular $(\Delta \varepsilon<0)$ to the field. For a simple nematic phase the torque can be calculated to be [23]:

$\Gamma_{\varepsilon}=-\frac{1}{2} \varepsilon_{0} \Delta \varepsilon E^{2} \sin 2 \phi \hat{y}$

Here $\varepsilon_{0} \approx 8.85 \cdot 10^{-12} \mathrm{C} / \mathrm{Vm}$ is the vacuum permittivity and $\phi$ is the angle of the director with respect to the electric field. The director is considered to be in the $x-z$ plane and the field is applied along $-\hat{x}$.

In a device or in a typical research sample cell there is however also a strong influence from the sample substrates, imposing the starting configuration (normally planar with a positive $\Delta \varepsilon$ liquid crystal). When an electric field is applied we thus typically get a competition between the dielectric contribution to the free energy, promoting a reorientation of the director, and the elastic contribution, counteracting any change from the initial planaraligned state. At weak fields the elastic contribution 'wins' and the director remains unaffected by the field, at strong fields it is the other way around and the sample is switched. The borderline case is called the Frederiks threshold after the Polish-Russian physicist Vsevolod Frederiks, who was the first to study the switching phenomenon in detail [1]. The switching process is nowadays called the Frederiks transition. The anisotropy of liquid crystals is also reflected in their magnetic and conductive properties as well as in their multiple viscosities, see e.g. [23] for details.

\subsection{Deformations and singularities in the nematic director field}

The undeformed elastic ground state of an achiral nematic is one where the director field is totally uniform, i.e. $\mathbf{n}$ points in the same direction everywhere throughout the sample. An elastic deformation in the case of a nematic results from a local reorientation $\delta \mathbf{n}$ of the director, leading to a director field distortion transmitted over a larger scale. Since distortions in $\mathbf{n}(\mathbf{r})$ cost energy, the nematic tries to reestablish the uniform director field, not by a force as in solid elasticity but by an elastic torque. The strength of the torque can be calculated by differentiating the elastic free energy. Neglecting surface effects, any generic deformation of $\mathbf{n}(\mathbf{r})$ can be described as a linear combination of three elementary elastic deformations, defined graphically in Fig. 6: splay, twist and bend. As a result the full elastic free energy of a bulk non-chiral nematic can be written

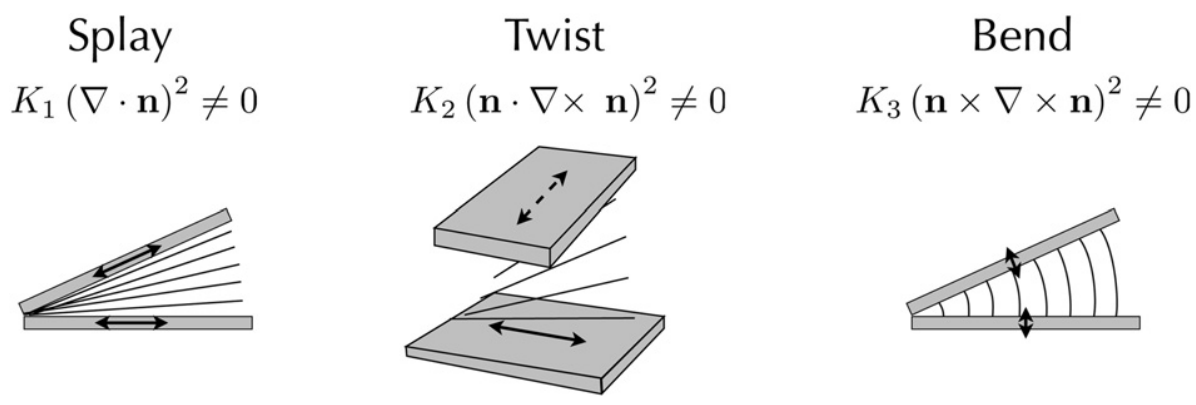

Fig. 6. Graphical definitions of the three elementary director field deformations splay, twist and bend, together with their corresponding terms in the elastic free energy and their respective elastic constants $K_{1}, K_{2}$ and $K_{3}$. The drawings illustrate how each deformation can be produced in practice by encapsulating a nematic between flat substrates, the insides of which are prepared such as to ensure uniform planar or homeotropic director orientation at the substrate (the preferred director is indicated with a thick double-headed arrow). Redrawn after de Gennes and Prost [23]. 
as a sum of three terms, each giving the total elastic free energy contribution due to splay, twist and bend deformations in the sample, respectively:

$G_{\text {deform. }}=\frac{1}{2} K_{1}(\nabla \cdot \mathbf{n})^{2}+\frac{1}{2} K_{2}(\mathbf{n} \cdot \nabla \times \mathbf{n})^{2}+\frac{1}{2} K_{3}(\mathbf{n} \times \nabla \times \mathbf{n})^{2}$

This expression is referred to as the Oseen-Frank expression for the free energy, after the Swede Carl Wilhelm Oseen and the Englishman Sir Charles Frank.

Each deformation is described by the appropriate combination of the vector operators divergence $(\nabla \cdot \mathbf{n})$ and curl $(\nabla \times \mathbf{n})$ squared and the energy cost for a certain deformation is proportional to the respective elastic constant, $K_{1}$ for splay, $K_{2}$ for twist and $K_{3}$ for bend. These constants are very important material parameters, critically dictating the behavior of a nematic. They have the dimension of a force, the magnitude typically in the $\mathrm{pN}$ range, and they must be positive (required for stability). Their values are so small that thermal motion induces considerable fluctuations in $\mathbf{n}(\mathbf{r})$ in a bulk nematic at room temperature. Often $K_{1}$ and $K_{2}$ have about the same value whereas $K_{3}$ is about twice that value. It can be convenient to approximate them all equal to a single value $K$, the so-called 'oneconstant approximation'.

The director fields shown in Fig. 6 are all smooth and locally defect-free despite their deformed state. If we prepare a nematic sample with degenarate planar alignment we will however almost always have defects in the director field, i.e. points where $\mathbf{n}(\mathbf{r})$ is not defined. Such a defect is called a disclination. Some examples of disclinations are shown in Fig. 7 together with exemplifying polarizing micrographs of how the most common ones may appear visually. The drawings are two-dimensional as is the plane of observation in the microscope. The lines illustrate $\mathbf{n}(\mathbf{r})$ in the sample plane and the black dot indicates the disclination itself (the point where the director is undefined).

As can be seen in the figure, a disclination can be given a defining parameter $s$, giving it a certain magnitude and sign. This resembles electrical charges and, in fact, the interaction of disclinations is in many respects analogous to electrostatics, with likesigned disclinations repelling each other whereas disclinations of opposite signs attract. Disclinations with $|s|\rangle 1$ are rarely observed since the energy density in the surrounding field of a singularity increases as $s^{2}$. Energy is thus generally gained by splitting an $|s|=$ 2 disclination into two $|s|=1$, or one $|s|=1$ into two $|s|=1 / 2$. In three dimensions the 2D drawings discussed here correspond either to a defect line through the bulk (valid for half-integer disclinations) or to a point defect stuck at the surface ( $s$ integer). For a richer discussion of these issues, see e.g. the text book by de Gennes and Prost [23].

Disclinations are important in today's research on liquid crystals foremost for two main reasons. In one case the topology of the sample requires disclinations to form, e.g. in planar-aligned droplets or shells of liquid crystal. In the second case we create disclinations by adding colloidal particles to the liquid crystal, such as glass or polymer beads or isotropic liquid droplets. Both cases will be discussed below.

The elasticity theory described above was developed for nematics but it can be extended and modified to phases with partial positional order. For each particular phase one must however take into account the special restrictions imposed by its symmetry. It is for instance relatively easy to realize that two of the three elementary deformations are not allowed in SmA due to its structure. Since the director also defines the layers through its double role as layer

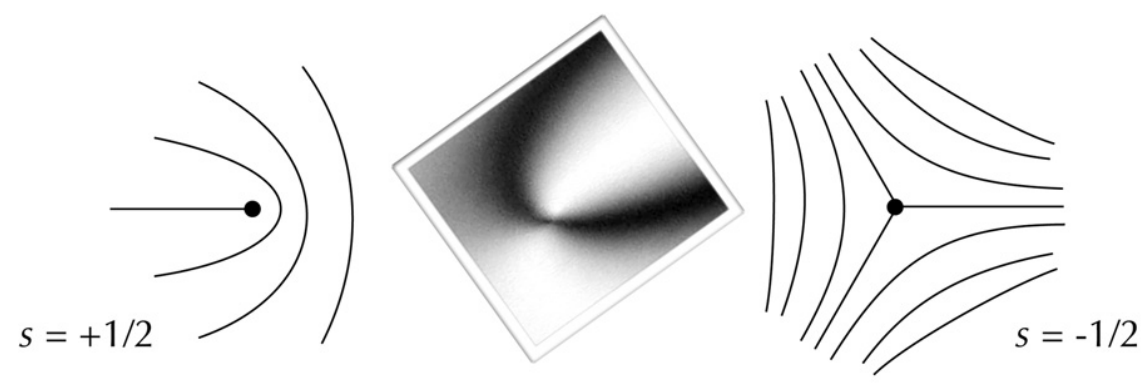

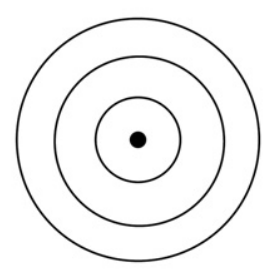

$s=+1$

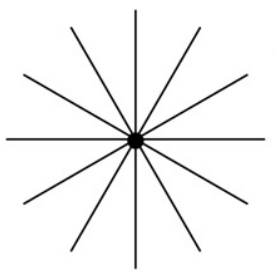

$s=+1$
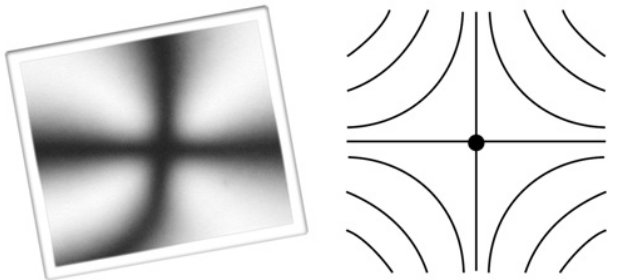

$S=-1$

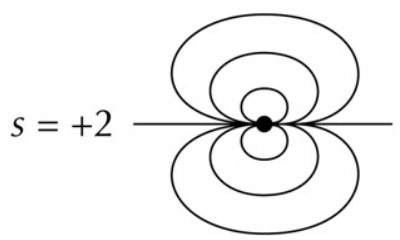

Fig. 7. Examples of disclinations of magnitude $1 / 2,1$ and 2 , of positive and negative sign, in a planar-aligned nematic. For the $s= \pm 1 / 2$ and $s= \pm 1$ cases, polarizing micrographs are provided as illustrations how these disclinations can be recognized in the polarizing microscope. Note that the sign of the disclination cannot easily be determined from a single micrograph. 
normal, a twist in the director field also entails a twist in the layer structure, something that would require a breaking up of the layers. This cannot happen in ordinary SmA phases and thus twist deformations do not occur in SmA. As for director bend, also this gives problems with the layer structure since a bend in $\mathbf{n}(\mathbf{r})$ effectively corresponds to a splay in the layers. The layer thickness would have to change constantly, a situation that does not occur in reality since the layer thickness is defined by the effective length and inclination of the molecules. We are thus left with only director splay as a possible deformation in SmA, corresponding to smectic layer bend.

Although SmC is less symmetric and thus in some sense more ordered than SmA (occurring at lower temperatures) it actually allows more fluctuations in the director field than SmA. This is because the addition of a tilting direction gives a new freedom for deformations which do not interfere with the layered structure, allowing $\mathbf{n}$ to bend as well as twist in SmC and SmC*.

We end this brief introduction to liquid crystal elasticity by pointing out that this is a continuum description: it relies fundamentally on the assumption that the liquid crystal's true discrete molecular nature can be neglected, the fluid being treated as a continuum instead. This assumption holds as long as the objects and phenomena under discussion have sizes that are very large on a molecular scale. Since this is indeed the case in most practical uses of liquid crystals, the elasticity theory is most often valid and extremely useful. There are however situations where the theory may break down, in particular in the research on nanoparticles in liquid crystals.

\section{New directions for applied and fundamental liquid crystal science}

In the remainder of this article we will highlight a few of the major current directions into which liquid crystal science is heading today. Other authors might have made a slightly different selection from the very rich playground that research on liquid crystals constitutes, but we believe that the examples given do represent some of the most exciting activities, and they certainly attract considerable interest across the international research community.

\subsection{Liquid crystals for organic electronics and energy conversion applications}

An area where the spontaneous long-range order of liquid crystals and the ease of controlling their alignment over large areas is very attractive is organic materials for semiconductor devices and energy conversion. In particular the use of liquid crystals for building efficient organic photovoltaic and transistor devices by selfassembly has been attracting attention for some time [7,24-27], but recently there were also impressive demonstrations of mechanoelectrical energy conversion utilizing the high flexoelectric coefficients of bent-core nematics [28-31]. Here we will first give a brief review of the activities in the former field and then we end this subsection with a snapshot of the state of the art of the latter.

The interest in organic electronics has been very strong on an international scale and over a relatively long period of time, and as a result a large number of aromatic molecules that are potentially useful for organic semiconductors or solar cells [32] have been designed. The single-molecule properties are however only one factor in determining the performance of a device employing these materials. In order to ensure high charge carrier mobility on a device scale, the molecules must be packed in a well ordered arrangement providing good overlap of the $\pi$ orbitals of the aromatic moieties. Long-range orientational and at least partial positional order allows the orbital overlap between adjacent molecules to extend over macroscopic distances, thus leading to the common picture of band-like conduction, cf. Fig. 8. The type of conduction is however of the hopping type. Amorphous materials are obviously inappropriate in this respect since the molecules are disordered already on a small scale. Crystalline structures might on the other hand seem to be ideal at first, but one must then remember that, for device application, the ordered arrangement must extend uniformly over distances corresponding to the interelectrode distances of the device. This typically ranges from some hundreds of nanometers to several microns. If the order breaks down locally, as in a grain boundary, the good conduction path is broken. Because it is very challenging to achieve perfect crystals of the desired materials over the distances required, this makes the route via the crystalline state less than optimal.

By using molecules that are suitable for organic electronic applications at the same time as they exhibit mesogenic properties, one can solve the problem of large-scale alignment in an elegant and simple way [24,25]. A liquid crystal phase is much easier to align than a crystalline phase and grain boundaries can be annealed rather easily. Moreover, they are highly suitable for solution processing. The most commonly employed liquid crystals in this context are the columnar phases of discotic mesogens, providing good charge carrier mobility and band-type conductivity along the

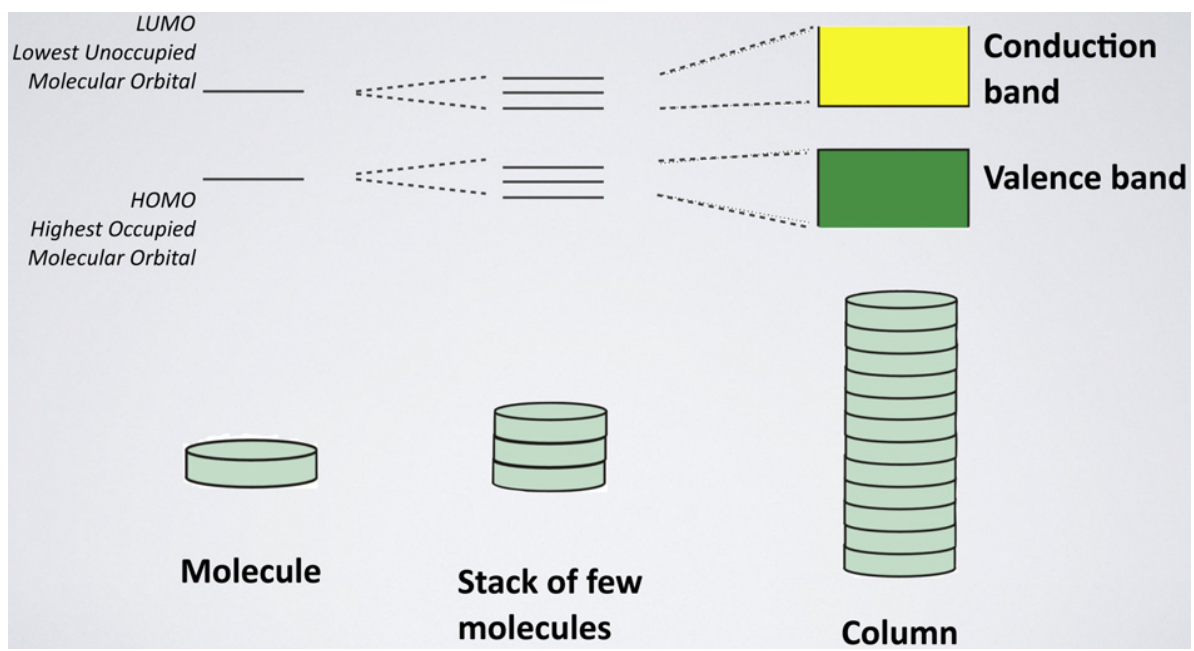

Fig. 8. Cartoon illustrating the transition from individual energy levels to energy bands as the extension of $\pi$ electron overlap grows along the columns of a columnar discotic liquid crystal phase. 
columns which thus effectively become a kind of 'molecular wire' [24-26]. But also smectic and nematic phases of rod-shaped mesogens have been explored in this context.

Hanna and co-workers functionalized molecules optimized for field effect transistor applications with alkyl chains, giving them smectic phases between the crystalline and isotropic states. By dissolving the material in an appropriate solvent and spin coating the solutions at a temperature where the pure material is in the smectic state, they achieved excellent films with very high uniformity and long-range order [33], cf. Fig. 9. If the material was instead spin coated at low temperature, such that a crystalline state forms when the solvent evaporates they achieved a rather inhomogeneous polycrystalline film with a rough surface. The result could be improved by raising the temperature to the liquid crystalline range after spin coating and thus annealing the film, but the film did not reach the quality of the film spin coated in the liquid crystalline state. Using a related non-functionalized material without the capability to form liquid crystal phases the results were even worse and the annealing option was obviously not available.

A drawback of using liquid crystalline systems for organic electronics is that the molecule mobility in the liquid crystalline state is still rather high. This leads to less strong $\pi$ - $\pi$-stacking and thus to a limited hopping conduction, considerably poorer than in a crystalline state. In general, the easier the phase is to align, the greater this problem. Nematics thus have the lowest charge carrier mobility among liquid crystal phases and even SmA and SmC phases have unsatisfactory values. Therefore, the most promising approach is generally to prepare the large-scale ordered arrangement in the liquid crystalline phase, in which the alignment can be controlled easily, and then quench the system to lower temperatures so rapidly that crystallization is prevented, instead giving a glassy state in which the liquid crystalline order is frozen in. In this way the good long-range order with little or no defects that can be achieved by processing in the liquid crystalline state can be combined with small molecular fluctuations, and hence high charge carrier mobilities over long distances can be expected.

An organic solar cell consists primarily of a heterojunction between an electron-donating and an electron-accepting material. The energy provided when a photon is absorbed leads to the creation of an exciton, i.e. a bound electron - hole pair. For the function of the solar cell the exciton should diffuse toward the interface where it can dissociate into free charge carriers in the two respective materials, that can then be harvested as an electrical current. In addition to the charge carrier mobility, already discussed, a key parameter for the photoelectrical conversion efficiency of organic solar cells is the exciton diffusion length, i.e. how
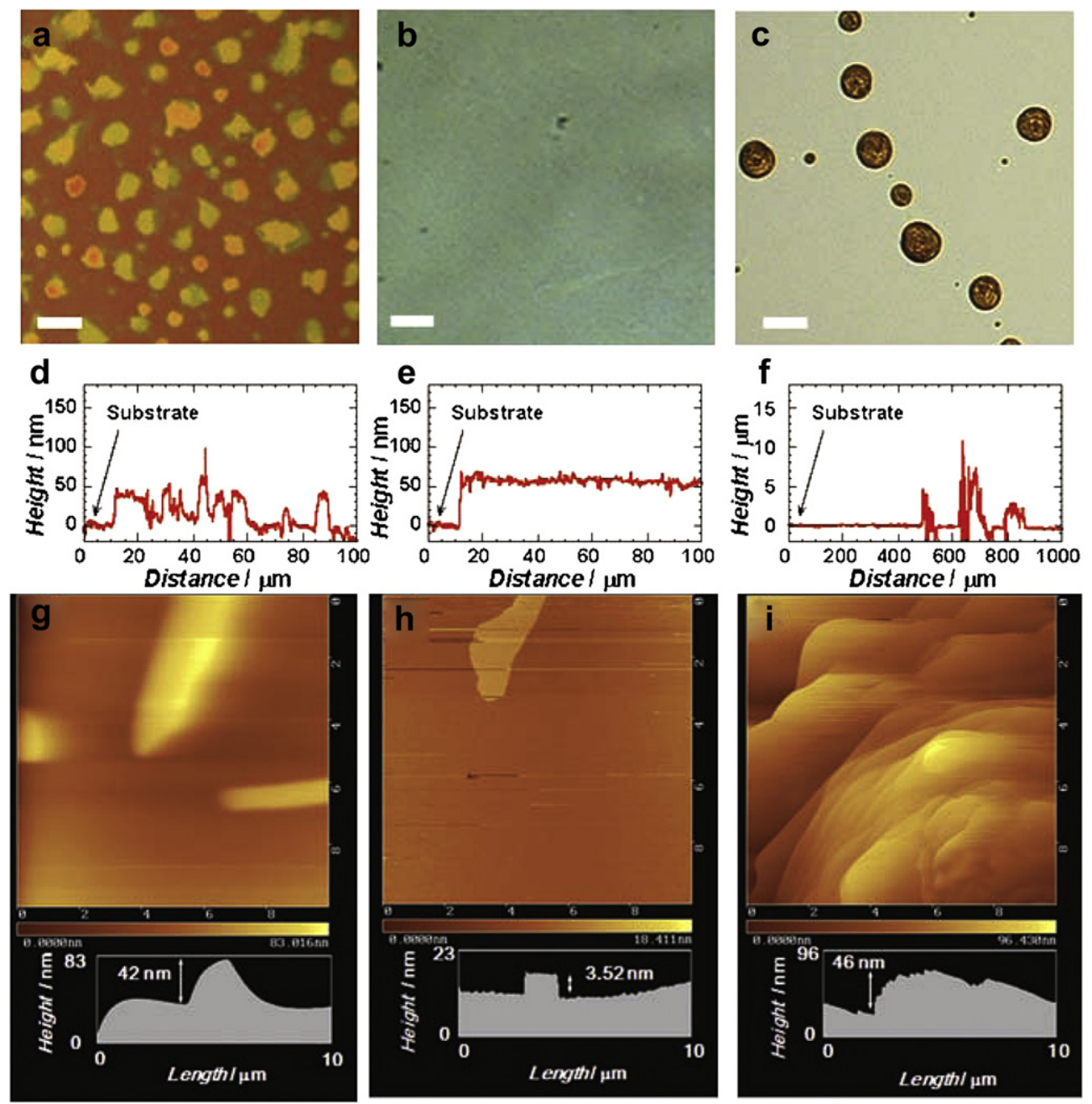

Fig. 9. Polycrystalline films of a doubly alkyl terminated terthiophene spin coated from isotropic solutions at different temperatures; a, $d$ and g: temperature of crystalline phase; $b$, e and h: temperature of smectic liquid crystalline phase; $c$, f and i: temperature of isotropic phase. Optical microscopy textures (a-c, scale bar in a-b: $10 \mu \mathrm{m}$; in c: $100 \mu \mathrm{m}$ ), confocal laser scanning microscopy profiles (d-f), AFM topographical images and corresponding height analyses ( $g-\mathrm{i}$ ). Note the very different vertical height axes in the latter diagrams for the different samples. From reference [33]. Copyright Wiley-VCH Verlag GmbH \& Co. KGaA. Reproduced with permission. 


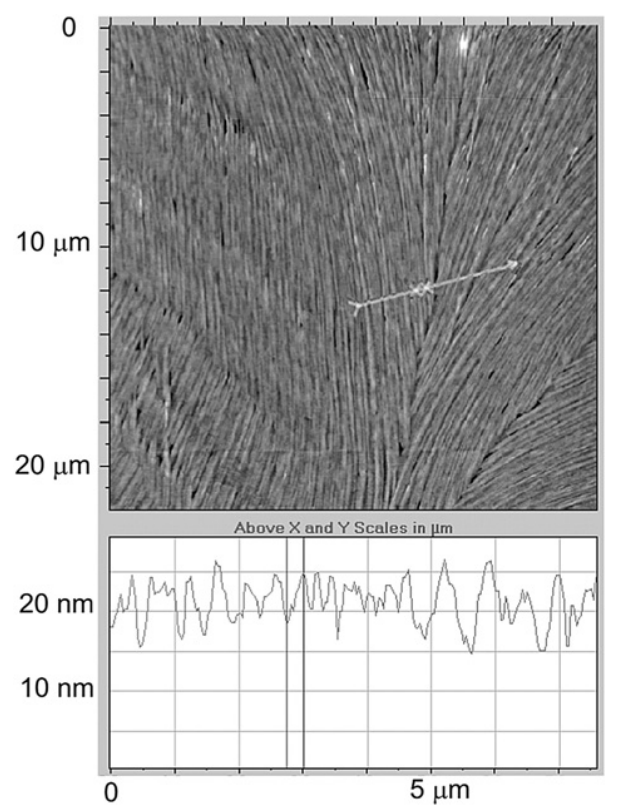

Fig. 10. Atomic force microscopy images of a sample of the discotic liquid crystal HAT5 spin coated from toluene solution, forming fibers organized in a nematic-like ordered arrangement. From reference [36].

long an exciton can travel within the material before the electron and hole recombine. The distance from the exciton creation site to the heterojunction must be smaller than the exciton diffusion length in order to harvest the energy. Discotic columnar liquid crystals have turned out to have particularly high values of this crucial parameter ( $>70 \mathrm{~nm}$ [7]) as well as high charge carrier mobility (1.4 $\mathrm{cm}^{2} \mathrm{~V}^{-1} \mathrm{~s}^{-1}$ [34]), and this has led to a strong focus of attention the last years on using these liquid crystals for photovoltaics $[7,26]$.

These materials clearly hold promise, as demonstrated with experimental and theoretical work, but important challenges still remain to be addressed. It is for instance not trivial to ensure the alignment of the columns that is required for a particular application. For a photovoltaic device based on discotic liquid crystals, one must create a uniformly homeotropic-aligned heterostructure of two different columnar phases, where one is designed for good electron-donating properties, the other one for being a good electron acceptor. This is quite challenging, but recently some hopeful reports appeared, suggesting that careful molecule design and optimization of the device preparation strategy may lead to the passing also of this hurdle, see e.g. [35].

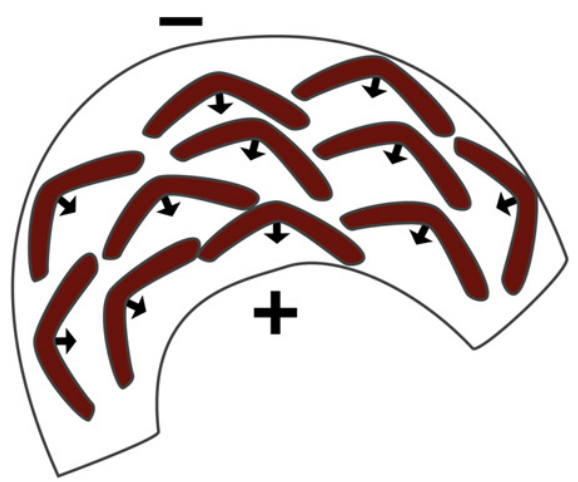

While homeotropic alignment is required for discotic liquid crystal solar cells, for an organic field effect transistor (FET) based on discotic phases the columns should lie in the sample plane and be aligned uniformly along a specific direction. This is perhaps even more challenging, because traditional alignment techniques such as rubbing of polyimide coating or photoalignment have little or no impact on the orientation of the director in discotic liquid crystal phases. To this end, an experiment by Scalia and co-workers may however show some promise [36]. They spin coated a toluene solution of the commonly used columnar phase-forming discotic material hexakis(pentyloxy)-triphenylene, abbreviated HAT-5 (Fig. 2c), and studied the produced films by polarizing microscopy and atomic force microscopy (AFM). The AFM study revealed very interesting textures, cf. Fig. 10, highly reminiscent of nematic director fields with a few disclinations between fairly large areas within which fibrils of columns were uniformly aligned. The authors proposed that the good alignment could be an effect of a lyotropic nematic phase of short disc columns forming during the spin coating process, as the solvent quickly evaporated. The director of this columnar-nematic phase would then align primarily along the radial shear flow active during the spin coating process. It is noteworthy that HAT-5 does not exhibit a nematic phase on its own, hence the proper combination of solvent concentration and temperature (note that the temperature rapidly cools during spin coating due to the evaporation of solvent) would be critical for obtaining the nematic order. Further investigations are clearly needed to confirm the exact origin of the uniform column alignment but this technique may be promising for aligning discotic columnar phases uniformly over areas that are very large on the scale of typical organic FET structures.

More recent and much less explored is the concept of harvesting mechanical motion as electrical energy using liquid crystals that display high flexoelectric and/or piezoelectric coefficients. The idea was proposed in 2009 by Antal Jakli and co-workers at Kent State University, USA [28,30,31]. The principle is illustrated in Fig. 11 for the case of a nematic phase made out of bent-core molecules. Such phases display the highest flexoelectric coefficients known so far. If the container of the liquid crystal bends, these molecules will pack more efficiently if they all bend in the same direction. Since they have a high polarization along the bend direction, bending of the sample thus polarizes it, and a surface charge results. Flexing an electrode-equipped sample back and forth then generates an alternating current.

Under practical application conditions liquid crystals are actually found to outperform many solid state alternatives in terms of energy conversion efficiency [28], since one must take the maximum strain gradient into account (which is substantially higher for the liquid crystal case) and as the liquid crystal-based materials can be made very thin, ensuring high efficiency. For

Fig. 11. A bent-core liquid crystal confined in a flexible container will produce a flexoelectric surface charge due to the improved packing efficiency when the bend of all molecules is along the macroscopic sample bend direction. If the sample is flexed back and forth and electrodes are attached to the outsides, an alternating electrical current can be picked up. 
experiments the Jakli team contained the bent-shaped mesogen phase between flexible substrates or inside an elastomer, allowing them to vary the macroscopic sample shape between convex and concave. Indeed, this flexing resulted in a current that was easily detectable by fitting the sample with two brass electrodes and connecting them to a standard oscilloscope. It will be very exciting to study the further development during the next few years of this very recently introduced concept, with considerable potential for new low cost means of energy harvesting.

\subsection{Liquid crystal templating for creating nanostructured materials or ordering nano- and microparticles}

With today's focus in materials science on nanostructured materials the ability of liquid crystals - thermotropic as well as lyotropic - to self-assemble into structures that exhibit specific nanoscale arrangements extending in an ordered fashion over a much longer range is extremely attractive. Several approaches have been devised to take advantage of the liquid crystalline order for generating new functional materials, either by making the liquid crystal-generated order permanent via polymerization, gelation or glass formation, or by preparing a liquid crystalline sample such that a regular array of defects forms, which can then be used e.g. for positioning nano- or microparticles over large areas. Here we briefly review the current state of the art of these two main approaches.

\subsubsection{Solidifying liquid crystal phases for creating regular} nanoporous solids

One of the most important current examples of how liquid crystals are used as templates is the production of nanoporous silica materials for catalysis applications from or via lyotropic hexagonal or cubic liquid crystal phases [37]. Very often the term 'mesoporous' is used instead, following a non-metric terminology common in this particular research field, but we here use 'nanoporous', reflecting the fact that the pores are in the nanometer size range. An organosilicate compound such as tetraethylorthosilicate (TEOS) is added as a silica precursor to the lyotropic phase and this is then hydrolized into $\mathrm{SiO}_{2}$. As the reaction takes place in the aqueous channels of the lyotropic liquid crystal host phase the produced silica adopts the internal shape of the nanostructured template, cf. Fig. 12. The soft and sensitive liquid crystal structure is thereby transferred to a hard and durable inorganic copy. Afterward the surfactant is removed by thermal decomposition (calcination), leaving only the nanostructured silica which now has the hexagonal or cubic structure imprinted by the liquid crystal.

In some cases it is of advantage to follow a different approach for solidifying the lyotropic structure, instead replacing the ordinary surfactants with surfactants with reactive headgroups [38-41]. In this way one can polymerize the surfactant component of the lyotropic liquid crystal and rather than making an inorganic copy of the template, the liquid crystalline structure is thus fixated by turning it into an organic solid. The resulting materials are of interest for instance in water filtration applications or as synthetic biodegradable materials for medical applications.

Also thermotropic liquid crystals can be very useful as templates in various contexts, realizing a solid either by polymerizing the liquid crystalline phase $[42,43]$ or by polymerizing another material inside the liquid crystal $[44,45]$. A particularly beautiful example of the latter approach is the successful production of helical polyacetylene fibers by polymerizing acetylene gas in a chiral nematic reaction field, a strategy pioneered by Hideki Shirakawa and Kazuo Akagi and co-workers at Kyoto University, Japan [46]. The cholesteric liquid crystalline environment forces the polyacetylene chains to twist as they grow, into fibers that mimic the structural features of the helical liquid crystal phase. The resulting fibers exhibit physical properties that can be quite unique, in particular in terms of their magnetoresistance. This has triggered much interest in areas very far from liquid crystal research [47]. The chiral liquid crystal reaction field is critical for the success in the preparation of this new material.

An interesting recent extension of liquid crystal structure formation, related to lyotropic liquid crystals in many respects but not involving a solvent, is the class of polyphilic mesogens [8,48-55]. With these molecules, that are amphiphilic with at least
A

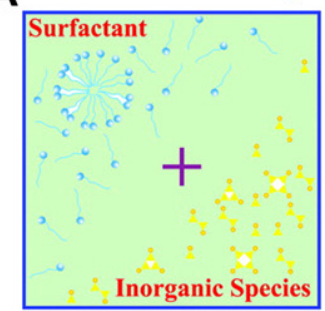

Cooperative Nucleation

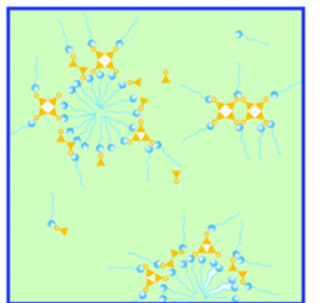

Cooperative Aggregation and Phase Separation
Mixture of Solution and Precipitation
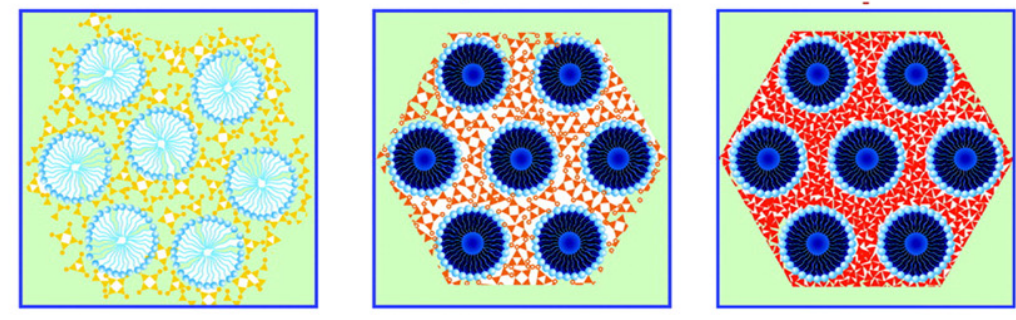

Liquid Crystal Formation with Molecular Inorganics
Further Polymerization and Condensation of Inorganics
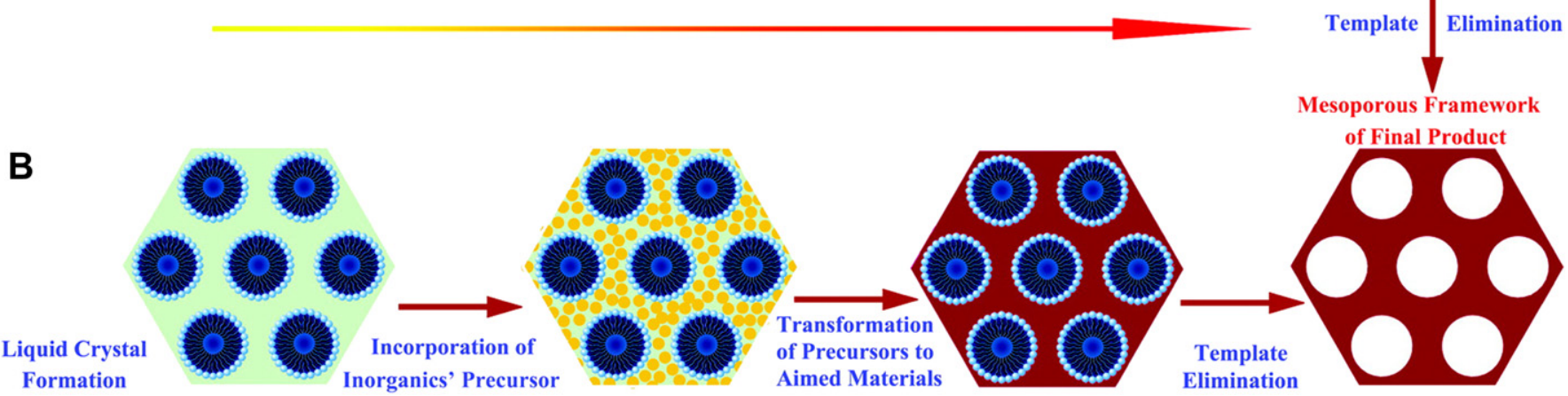

of Final Product Inorganics' Precursor Aimed Materials 
three, rather than two, sections having different preferred environments (for instance one perfluorinated, one hydrophilic and one aromatic section) a whole range of new structures have been found to form via self-assembly. At the moment the research on these new materials is purely academic, but the complexity of these new phases is beginning to be elucidated. The time may thus be ripe for exploring them for different types of applications, where an obvious route to explore is that of templating from these new nanostructures. Compared to lyotropic liquid crystals a huge advantage with the polyphilic thermotropics is that they can generally be quite easily aligned over macroscopic areas.

\subsubsection{Synthesizing nanoparticles in liquid crystalline templates}

Several groups have used the self-organized structures of liquid crystals as 'nano reactors' for synthesizing very well-defined nanoparticles with desirable shapes. In particular lyotropic columnar phases are useful for synthesizing e.g. semiconductor nanorods [56-62] but other liquid crystal phases can be used for synthesizing nanoparticles with other shapes [63-67].

Among the key advantages with this technique compared to standard methods for synthesizing non-spherical nanoparticles are low dispersity, a relative ease of tunability of the geometrical features, and the fact that very high aspect ratio particles can be achieved by carrying out the synthesis within the very long channels of the micelles in a hexagonal columnar phase. By tuning the composition of the phase, often including an oil component in addition to the amphiphiles and water that form the starter kit, the inner diameter of the channels can be varied over an order of magnitude (1-10 nm) while their length can be in the centimeter range or even longer. Another beneficial aspect is that it is easy to remove the components of the lyotropic phase template. This is in contrast to methods where solid templates are used, which typically require etching by strong and/or harmful acids.

Obviously one needs also precursor materials in addition to the basic constituents of the liquid crystal phase. These are often organometallic salts that act as precursors for the nanoparticle synthesis. It is crucial to recognize that these salts will normally become a component of the liquid crystal phase as well and their presence will almost always have some influence on the selfassembly processes leading to the liquid crystal phase formation. One thus generally needs to modify the recipes for forming pure liquid crystal phases, giving the precursor materials active roles in the structure formation. In some cases the influence from the salts would simply be too strong at the concentrations required for forming interesting particles and one must then find special solutions. A smart approach employed by dos Santos et al. is to incorporate colloids among the precursor materials [62]. This allows a relatively high degree of loading with inorganic material with minimal influence on the lyotropic phase.

\subsubsection{Colloidal particles organized by liquid crystals and liquid crystal phases from colloids}

A different way of exploiting the liquid crystalline order, that does not necessarily involve fixating the structure into a solid, is to introduce colloidal particles in the liquid crystal. The latter then functions as a very special continuous phase, acting in unique ways on the dispersed particles which can be in the nano- to micrometer size range and of a variety of shapes. There has been a continuously growing interest in combining particles with liquid crystals over the last 15 years, and a number of interesting phenomena have been demonstrated, involving polymer or silica microbeads, carbon nanotubes, or semiconductor or metal nanorods or -spheres.

The liquid crystal can organize the dispersed particles in various ways and employ somewhat different mechanisms, depending on the particle size, shape and how their surfaces interact with the liquid crystal. What all studies have in common, however, is that the particles in some way disturb the liquid crystal director field, which therefore exerts forces or torques on the particles in order to promote a configuration of them where the distortion is minimal. This can lead to an alignment of anisometric particles as well as to the organization of particles into regular clusters.

Among the most straight-forward approaches of this kind is the alignment of rod-shaped particles along the nematic director field. The first particle type to be studied in this way was magnetic nanorods, initially considered theoretically by Pierre-Gilles de Gennes and Françoise Brochard [68]. In recent years the first nanoparticle to receive considerable attention as dopant to liquid crystals was the carbon nanotube [69-81]. Single- as well as multiwall carbon nanotubes have been successfully dispersed in thermotropic as well as lyotropic liquid crystals, primarily of nematic type, and the alignment of the tubes along the nematic director field was verified by various techniques. Initially only indirect proof of the alignment could be provided $[69,70]$, but somewhat later direct in-situ verification of the nanotube orientation by means of polarized Raman spectroscopy confirmed without doubt the alignment effect $[82,83]$. The experimental work was also complemented by a number of rigorous theoretical treatments [84-87].

A rather severe problem with the carbon nanotube - liquid crystal composites is to maintain a sufficiently good dispersion of the nanotubes. Carbon nanotubes are notoriously difficult to disperse in any medium and in liquid crystals long-term stability of the dispersion is extremely difficult to achieve. All initial work was done with thermotropic liquid crystal hosts and only trace amounts of carbon nanotubes were dispersed in order to avoid severe nanotube aggregation. The first successful way of drastically raising the nanotube concentration was to switch to lyotropic liquid crystal hosts, where the option to use surfactants for stabilizing the nanotube dispersion allowed a much greater concentration of the nanoparticle guests $[78,83,88,89]$ Particularly high nanotube loading was possible by using two different ionic surfactants of opposite charge, one for dispersing the nanotubes and the other for building the liquid crystal phase. In this way Scalia et al. succeeded in reaching such high nanotube loading at such good alignment that the composite behaved like a liquid linear polarizer [90,91], cf. Fig. 13: as the nanotubes absorb light polarized along the tube axis, the composite became darker for polarization along the director than perpendicular to it. This was the first time that nanoparticle alignment by a liquid crystal could be verified by the naked eye. The composites also acquire very interesting viscoelastic properties, in particular the ability to form very long and slender filaments in

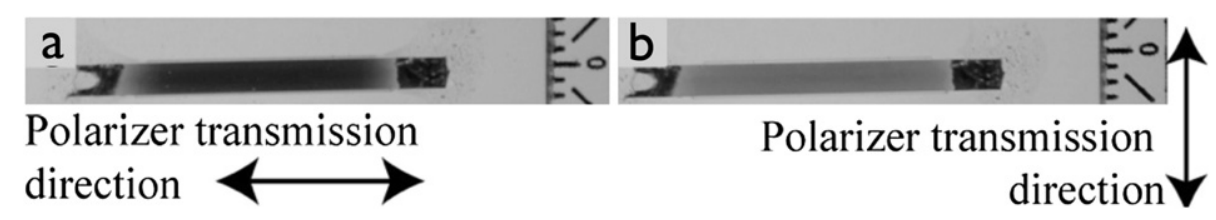

Fig. 13. A capillary containing a lyotropic liquid crystal phase aligned with $\mathbf{n}$ along the capillary axis and containing 0.2 wt.\% well-dispersed single-wall carbon nanotubes. The uniform alignment of the nanotubes, present at such high concentration, turns the composite fluid into a polarizer, as demonstrated by viewing the capillary through a polarizer film with its transmission direction along (a) and perpendicular (b) to the capillary. From reference [90]. Reproduced by permission of The Royal Society of Chemistry. 
which the nanotubes are highly ordered [92]. A number of studies on dispersion of nanotubes in lyotropic liquid crystals have followed [93-98], confirming the versatility and usefulness of the approach. An important recent achievement is the polymerization of the lyotropic host phase in order to turn the nanotube - liquid crystal composite into a solid, with maintained uniform alignment of the nanotubes [96].

There is however considerable potential in using also thermotropic liquid crystals as the host for nanotube dispersion [99-102], in particular due to the ease of aligning and reorienting thermotropic nematics, and there is thus a motivation for improving the dispersion stability also in thermotropic hosts. In fact, although long-term stability is a serious problem, the first systematic study of carbon nanotube dispersion in thermotropic liquid crystals [103] revealed that thermotropic hosts are actually among the best organic solvents for carbon nanotubes in terms of a quick reduction of initial aggregate size. The problem is to ensure exfoliation of bundles to single tube level and to maintain long-term dispersion stability, very difficult since no surfactants can be added for stabilizing the dispersion. An important result of this study was that there are considerable differences between different nematics in terms of their suitability as hosts for carbon nanotubes, the commonly used quartenary mixture E7 and the negative dielectric anisotropy nematic MBBA coming out as the best hosts of those investigated [103]. A promising recent development is the introduction of tailor-synthesized hybrid molecules, acting similar to surfactants in aqueous solution, but here with one end optimized for carbon nanotube adhesion whereas the other is made for thermotropic liquid crystal compatibility. This is a very new research thrust but some promising results have been presented [104-106].

There have also been observations of positive effects of carbon nanotubes on the liquid crystal [71-74,80,101,107,108], even including the function of carbon nanotubes as chiral dopants $[109,110]$, but as the liquid crystal in these cases plays a passive role we do not further discuss these issues in this review. Almost all work has been done with nematic hosts of rod-like mesogens, primarily due to the difficulties in dispersing nanotubes in more ordered and thus much more viscous liquid crystal phases. A rare example of a study of nanotubes dispersed in a chiral smectic liquid crystal was published by Lagerwall et al., the success in dispersion being due to a two-step process where the nanotubes were first dispersed in a nematic phase that was then mixed with a strong smectogenic mesogen [100]. The nanotubes turned out to have a drastic influence on the phase sequence of the smectic host but the dispersion was still not sufficiently good to investigate if switching of the smectic liquid crystal director might have an effect on the nanotubes. Kumar and co-workers succeeded in dispersing chemically functionalized nanotubes in thermotropic discotic hosts $[3,111,112]$, but also here little efforts were done to investigate the effects of the host on the organization of the nanotubes.

Rather than doping a liquid crystal host with the nanotubes one can in fact also get the nanotubes themselves, suspended in an isotropic liquid host, to form a lyotropic liquid crystal phase, provided sufficient concentration and quality of dispersion [16,18,113-124]. This has the advantage that the nanotube concentration obviously is high, much higher than in the work relying on thermotropic hosts, but there are two major difficulties. First, even in isotropic solvents it is far from trivial to achieve sufficiently good dispersion at such high concentration of nanotubes required to get lyotropic liquid crystalline phases, and the groups driving this approach have therefore resorted to oxidation of the nanotubes for solubility in water [18,118-120], using super acids as the solvent [121-123] or taken great care in using efficient wrapping of pristine nanotubes by molecules such as DNA that stabilize the suspensions even at high concentration [16,113-117]. An example of the latter type is shown in Fig. 14. The second major difficulty is to ensure macroscopic orientation of the director in these phases over a long enough time scale that the aligned nanotubes can be put to use. Generally, shearing is the only way to align lyotropic phases of this type but as soon as the shear flow is removed the alignment is often lost. Recently the Poulin team has however shown some progress in the task of retaining the alignment even after drying a lyotropic liquid crystalline nanotube suspension [116] and this route thus holds promise for the future.

With the very strong current focus on graphene it was hardly unexpected when the first reports on liquid crystal phases with graphene came out. Like with carbon nanotubes some groups use liquid crystalline phases for aligning the graphene sheets [125,126] while others ensure sufficient concentration and dispersion quality of oxidized graphene flakes that they form liquid crystal phases on their own [19-21,127].

Carbon particles were the first but of course not the only nanoparticles that have been studied in combination with liquid crystals. Several studies have been devoted to the investigation of liquid crystalline phases containing gold [128-142], semiconducting [143-154] or silica nanoparticles [155-159] as well as magnetic [160-167] or ferroelectric [168-172] particles. While the extended aromatic structure of carbon nanotubes provides for beneficial interactions with typical mesogens, promoting particle solubility in a thermotropic host [103], the primarily hydrophilic character of many non-carbon-based inorganic nanoparticles makes an efficient capping agent promoting guest-host compatibility absolutely essential if metal or semiconductor nanoparticles should be efficiently dispersed in a thermotropic liquid crystal. Common choices for solving the dispersion problem are alkane thiols $[129,136,140]$ which bind covalently to a metal surface with the thiol head group, extending an alkyl chain more or less perpendicular to the particle surface. This improves the compatibility with a thermotropic host considerably, but it promotes homeotropic alignment of the mesogens surrounding the particle, hence one may expect the opposite alignment of alkane thiolfunctionalized nanorods compared to carbon nanotubes, i.e. perpendicular rather than along the liquid crystal director. The much lower aspect ratio of most inorganic nanorods (typically two orders of magnitude less than for carbon nanotubes) makes the alignment a slightly less serious issue. In fact, many studies deal with spherical gold or semiconducting quantum dot particles. Another alternative is to use a nonionic polymer such as polyvinylpyrrolidone (PVP) for aiding the dispersion [133].

Much of the work devoted to inorganic nanoparticles in liquid crystals is devoted to modification of the liquid crystal properties in its classic electrooptic device (e.g. display) application, e.g. for

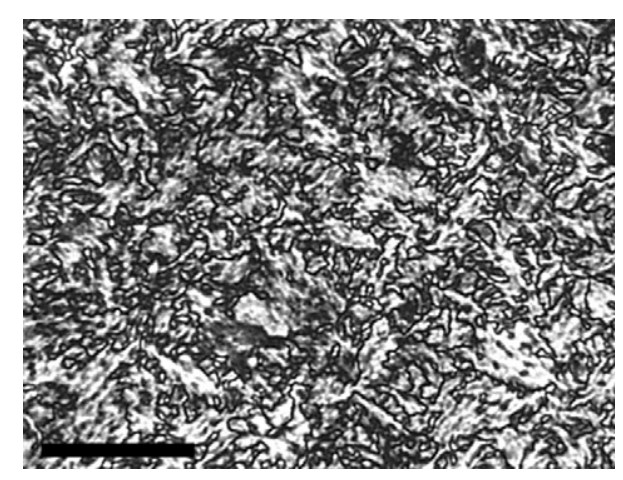

Fig. 14. Polarizing micrograph of a nematic liquid crystal phase from single-wall carbon nanotubes stabilized by DNA in water at 4 wt.\% concentration (scale bar: $50 \mu \mathrm{m})$. From reference [114]. Copyright Wiley-VCH Verlag GmbH \& Co. KGaA. Reproduced with permission. 
modifying switching time or alignment type [137,150,153,173], and is thus outside the scope of this review. From the standpoint of this article there are two lines of research in this field that are of particular interest. First, gold nanoparticles are attractive due to their plasmonic response but this depends also on the dielectric permittivity of the medium surrounding the nanoparticles. Therefore, if the continuous phase of the gold nanoparticle colloid is a thermotropic liquid crystal, the plasmonic response can easily and rapidly be tuned by changing the dielectric constant component experienced by the nanoparticles by switching the liquid crystal with an external electric or magnetic field $[132,174,175]$. A second thrust that may turn out to be very interesting is the effort to develop metamaterials using metal nanoparticles in liquid crystals, but this is considerably more challenging since complex nanoparticle shapes are required, little fluctuations can be accepted, and highly regular arrangements of the particles are sought for [176,177]. We will return to this issue in a moment.

Like with surfactant-stabilized carbon nanotubes lyotropic liquid crystal hosts can be quite advantageous in ensuring a high loading of metallic nanoparticles. For instance, using the same cationic surfactant-based lyotropic liquid crystal host as was used very successfully for carbon nanotube alignment by Scalia and coworkers, as described above, Liu et al. dispersed and aligned gold nanorods in nematic and hexagonal host phases [134]. Since the director can be macroscopically aligned and later realigned by magnetic fields in the nematic phase or shear flow in the columnar phase, the plasmonic response could be tuned by changing the nanorod orientation.

Some of the most beautiful work on particles in liquid crystals has dealt with sphere-shaped particles or droplets in the micro- rather than nanometer range. When dispersing e.g. spherical silica beads functionalized with capping agents that induce homeotropic director alignment of the surrounding liquid crystal, or glycerol droplets, generally inducing planar director alignment, each inclusion effectively becomes a unit strength topological defect in the director field due to the spherical symmetry of the director anchoring at its boundary. Because the total topological charge must be conserved, being zero in a flat surface, each positive unit defect in a flat sample cell introduced by an inclusion must be accompanied by a negative unit defect or a disclination ring generated next to it, turning the particle and its surrounding effectively into a topological dipole or quadrupole [178]. When several such multipoles are brought close to each other, e.g. by moving them with optical tweezers, the interactions between them, in many respects analogous to the interactions of electrostatic dipoles, result in chains or 2D patterns being generated and stabilized. Although beautiful experiments on the interaction of glycerol droplets with the nematic director field were conducted by Pawel Pieranski and co-workers already in the 1970s [179] the modern interest in this new class of responsive colloid took off after an elegant study published in 1997 of chains of surfactantstabilized water droplets in liquid crystals by Poulin et al. [180]. A number of groups have since achieved fascinating large-scale ordered arrangements of colloidal inclusions in nematic hosts based on the same principles, supported also by a number of theoretical treatments [158,181-206], and some groups have demonstrated the same type of phenomena in free-standing SmC films [207-212].

The research on colloidal inclusions in liquid crystals has so far been mainly of fundamental academic type but a few proposals for future application have been presented. For instance, Musevic and co-workers demonstrated the usage of such a system as a tunable optical grating [213]. The potential application that has probably attracted the most attention, however, is that of producing metamaterials by having the liquid crystal organize tailor-designed nanoparticles, such that composites with new and unique optical properties can be realized. The main goal is to produce negative refractive index materials functioning in the optical wavelength regime, which could be used for cloaking or perfect lensing [176,177,214-217]. This typically requires the positioning of nanoscale conductive rods and conductive incomplete rings (split ring resonators) in regular arrays over large distances.

Obviously, the research on liquid crystal-driven positioning of particles is still very far from reaching this goal. The particle size must be in the nanometer range, hence the work on micron scale particles must be scaled down (this may not be possible for spherical particles as one may reach the limit of the continuum description of the liquid crystal, below which the elasticity theory for the distorted director field breaks down), and the positioning of spherical particles is generally not of much use per se in this context. The successes in aligning metallic nanorods and carbon nanotubes (which can be metallic or semiconducting depending on the geometry of the graphene network forming the tube) is promising but it is still not sufficient. Control of position is also required, something which has not yet been achieved, and generally also the split ring resonators must be arranged in the required configuration. Finally, one must also ask if the generally rather strong fluctuations in liquid crystals are acceptable for the realization of metamaterials with acceptable loss levels.

An original approach to realizing liquid crystal-organized split ring resonators was presented by the Ljubljana team around Musevic and Zumer. They trapped gold nanoparticles in disclination lines surrounding micron sized silica beads that are organized into an array by the nematic continuum phase [193], cf. Fig. 15. In this case the nanoparticles (100 $\mathrm{nm}$ in diameter in this study) migrate into the very core of the defect rings that form around the larger colloidal particles, as this reduces the volume of liquid crystal within which the orientational order breaks down due to the disclination. This type of nanoparticle ordering, somewhat different from the previously described approaches, is also being explored for other purposes, in particular with the aim to expand the temperature range of liquid crystal blue phases. These are exotic liquid crystal phases characterized by a cubic lattice of disclinations that may form in case of very strong chiral interactions [218]. The phase is in fact isotropic due to the cubic symmetry of the lattice, but birefringence can be induced by applying an electric field, and the display industry currently has a keen interest in the possibilities of exploiting this effect in displays that could be very fast and require no alignment control [219]. However, the temperature range of blue phases is normally very small, typically about a degree, and the hope is therefore to make the disclination line lattice more permanent by filling it with nanoparticles. Some positive reports have appeared, both of experimental $[141,220]$ and theoretical $[203,221]$ nature, but so far the achievements are not comparable to the more developed approach of expanding the blue phase temperature range by polymerizing a reactive monomer additive within the blue phase [222].

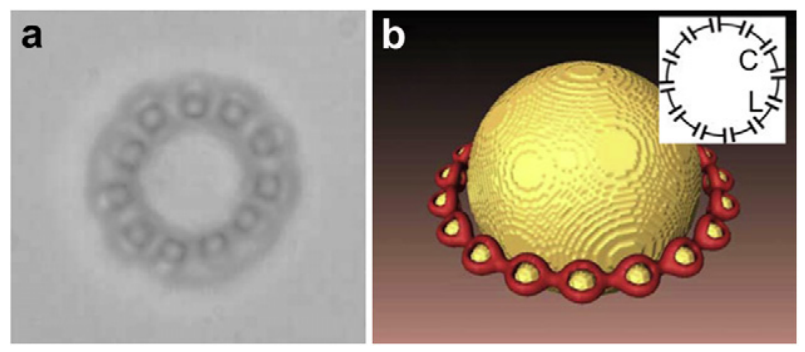

Fig. 15. (a) Colloidal gold particles ( $100 \mathrm{~nm}$ diameter) trapped into the Saturn ring disclination line surrounding a large spherical silica particle ( $1 \mu \mathrm{m}$ diameter) suspended in a thermotropic nematic liquid crystal host. (b) The calculated stable colloidal ring superstructure for this combination of colloids (inset shows a schematic of the corresponding electrical circuit). Reprinted figure with permission $\{$ http://pre.aps.org/abstract/PRE/v77/ i6/e061706\} \{from [193]\}. Copyright (2008) by the American Physical Society. 
An interesting variation of liquid crystal-based colloid ordering has been developed by the team of Hee-Tae Jung at KAIST, Korea, and Oleg Lavrentovich, Kent State University [223,224]. Rather than nematic hosts they work with smectics and they confine the phase inside narrow channels microfabricated in a silicon wafer. The channels are treated with a fluorinated polymer in order to ensure planar director anchoring along the side walls as well as at the channel bottom. The channels are open at the top and the air interface induces strong homeotropic alignment there. The conflict in alignment between the top and bottom forces the smectic phase to develop a regular array of toric focal conic defects, in each of which the layers curve around a central defect line. As in the cases discussed above, colloidal particles introduced to the liquid crystals can be trapped in these defect lines. In this way the team could distribute fluorescent particles along hexagonal patterns with very long-range fidelity solely by liquid crystal self-assembly [223]. The regular smectic liquid crystal defect array can also be used in general as a soft lithographic template [225] or as a basis for fabricating superhydrophobic surfaces [226]. By varying the cross section geometry of the micro channels and by replica molding the structures onto a photopolymerizable precursor material the group recently succeeded in broadening the concept [227]. They could now create a variety of defect patterns and in contrast to the initial works, which required rather special semi-fluorinated mesogens, they can now work also with the most commonly employed SmA material 8CB.

\subsection{Composite materials containing thermotropic liquid crystals as} functional additive

\subsubsection{Emulsions of cholesteric droplets for tunable omnidirectional} lasing applications

There is also a strong interest in colloids of the reverse type, where droplets of liquid crystal are dispersed in an isotropic continuous phase. The systems in focus range from dispersed nano droplets of lyotropic liquid crystal for drug or nutrient delivery, something we will come back to in Section 3.5, to droplets of shortpitch cholesteric liquid crystal for lasing applications. In the following we will describe this development in some detail, also taking the opportunity to briefly discuss the potential of cholesteric liquid crystals for lasing in general.
As with any emulsion, long-term stability can only be ensured by adding an emulsifier (e.g. a surfactant or polymer) to the continuous phase, that adsorbs at the interface between the liquid crystal and the surrounding medium. This reduces the interfacial tension between the two phases and it counteracts coalescence of the droplets. The typical choice for the continuous phase of an emulsion is water, with the added emulsifier being either a surfactant, often of ionic type, e.g. SDS or CTAB (see Fig. 2d-e), or a water soluble polymer such as polyvinylalcohol (PVA) or polyvinylpyrrolidone (PVP). In case of liquid crystalline droplets the choice of emulsifier is quite critical because it will influence the alignment of the director at the interface, and thereby throughout the droplet if it is not too large in diameter. A surfactant adsorbs at the interface with its non-polar alkyl chain extending radially into the liquid crystal phase, imposing a radial orientation of the director. This means that a surfactant-stabilized liquid crystal droplet will generally have homeotropic alignment. PVA or PVP, on the other hand, adsorb at the interface in a more or less random coil conformation, without actually entering the liquid crystal, and this leaves the liquid crystal with planar alignment, since this is the most favorable at an interface with a polar phase such as water.

As an alternative to using aqueous solutions of surfactants or polymers some researchers choose to use silicone oil [228,229] or glycerol $[230,231]$ as the continuous phase. This cannot give a longterm stable colloid, as nothing prevents the coalescence of droplets and as the interfacial tension is still non-negligible (although not as high as with water) but on a time scale of hours to days such a system can be sufficiently stable for experiments. The beauty of this approach is the simplicity in preparation. For instance, Humar and Musevic used simple hand stirring of a cholesteric liquid crystal in glycerol to prepare an emulsion with droplets of diameter in the 15-50 $\mu \mathrm{m}$ range [230]. They used cholesteric mixtures with different composition to tune the helical pitch over a large range, the most interesting having a pitch of 300-400 nm. With such short-pitch the droplets show selective Bragg reflection in the visible range along the droplet radius. This is attractive because the helix of a cholesteric or other chiral liquid crystal showing visible reflection can act as a resonant cavity for mirrorless lasing if one adds a fluorescent dye with emission wavelength tuned to the reflection band of the cholesteric [232-235]. Indeed, by adding

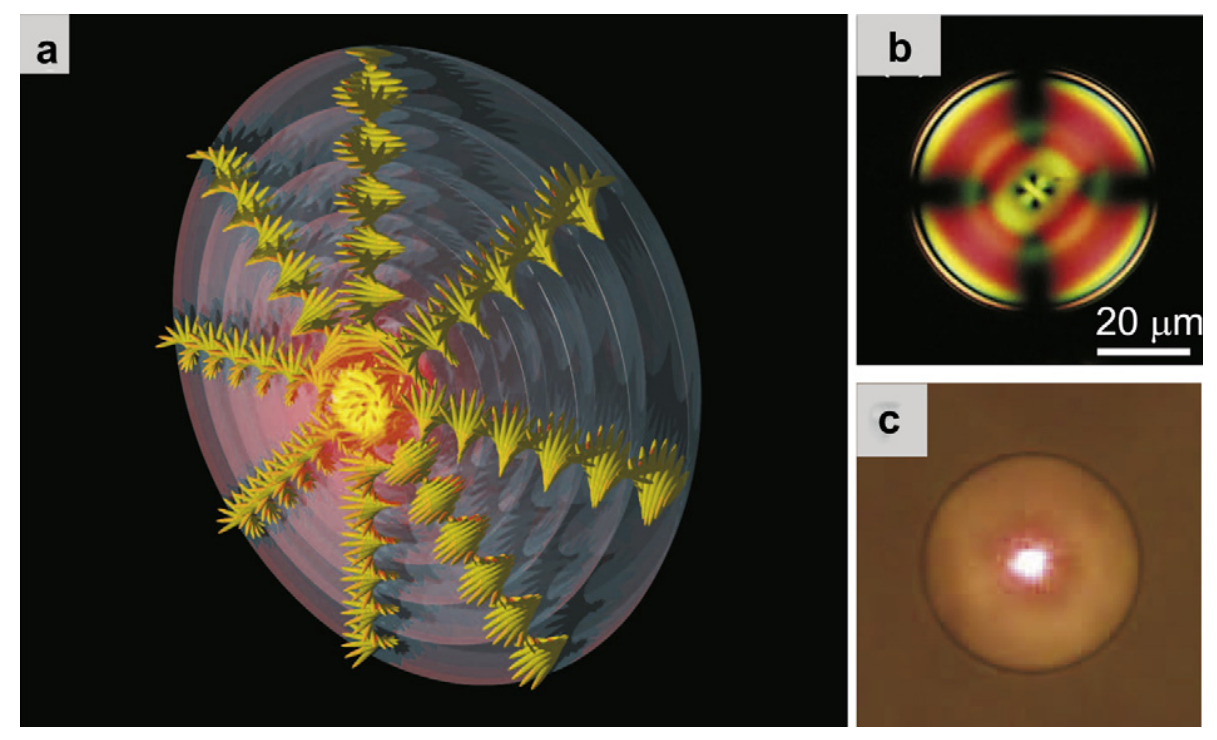

Fig. 16. (a) Schematic of the cholesteric droplets functioning as a medium for mirrorless lasing. (b) Polarizing microscopy image of a short-pitch cholesteric droplet reflecting red light. (c) Lasing from the droplet when pumped with an external pulsed laser source. Adapted with permission (Copyright 2010 Optical Society of America) from reference [230]. (For interpretation of the references to color in this figure legend, the reader is referred to the web version of this article.) 
a small amount of the laser dye 4-dicyanomethylene-2-methyl-6(p-dimethylaminostyryl)-4H-pyran (DCM) and pumping the colloid with a pulsed frequency-doubled Nd:YAG laser, lasing was achieved from the droplets at about $600 \mathrm{~nm}$, cf. Fig. 16. What makes this demonstration particularly interesting is that the spherical geometry of the cholesteric droplets leads to omnidirectional lasing, i.e. the laser light is emitted radially in all three dimensions. At the same time, the ease in tuning the cholesteric pitch, e.g. by changing the temperature, allows for some tuning of the lasing wavelength, although the range is limited by the requirement to match the emission wavelength of the laser dye.

The preparation procedure is attractive in its simplicity but the method suffers from lack of long-term stability and it does not offer very good control of droplet diameter. A fairly large dispersity in the droplet size is also to be expected. For improved control of the colloid characteristics it may thus be preferable to use more standard emulsion preparation techniques, typically dispersing the liquid crystal in an aqueous continuous phase, stabilizing the emulsion with added polymer (surfactants would not be useful here since the radial orientation of the cholesteric helix requires planar alignment). Still, the stability of such a system may not be satisfactory, a situation that motivated Landfester and co-workers in their work on 'mini emulsions' of oil phases in water prepared by ultrasonication to develop a smart trick [236]. They added a small amount of hexadecane to the non-polar phase, acting as a "super hydrophobe". Its very low solubility in the aqueous continuous phase creates an osmotic pressure directed into the droplet interior that counteracts the Laplace pressure, which promotes droplet coalescence. They reported a dramatic improvement of the emulsion stability in this way, and they even tested the concept successfully on liquid crystal droplets [237]. However, their study dealt with non-chiral nematic droplets of sub micron diameter. If one wishes to apply their methodology to the case of cholesteric droplets for lasing, which must be some two orders of magnitude greater in size, one would thus need to reduce the quantity of stabilizer in order to match the much smaller surfaceto-volume ratio of the larger droplets. It is also probably advisable to use a less intense dispersion method than ultrasonication, which typically yields very small droplet size. A nice alternative if the equipment is available is to use a specially designed microfluidic system to produce droplets of controlled size, an approach that has enabled quite beautiful studies of non-chiral nematic liquid crystal droplets under varying conditions [238,239].

A general problem with lasing from dye-doped chiral liquid crystals is the fact that continuous wave (CW) lasing has not yet been achieved. This may have several causes, the two most probable being heating of the liquid crystal such that its pitch changes too much or even to the extent that a phase transition into isotropic liquid occurs, and the limited stability of the organic dyes (inorganic dyes generally have too poor solubility in the liquid crystal). In this respect the droplet-based liquid crystal laser may offer a solution, provided that they are suspended in a low-viscous continuous phase that can easily be pumped through narrow channels. By incorporating the cholesteric droplet colloid in a microfluidic system it should be possible to continuously pump the colloid past the area exposed by the pumping laser, such that no single cholesteric droplet is exposed to the CW pumping radiation longer than it can support. As new droplets continuously enter the active area of the system a quasi-CW laser output might be achieved.

The future development of this very new concept of applying a liquid crystal emulsion will be highly interesting to follow. The initial results have already attracted much attention in the photonics community [240], hence there is a strong driving force for taking the concept to a stage where practical application is viable. If the remaining obstacles can be overcome, this would allow a very cheap fabrication of tunable 3D lasers of an entirely new type.

\subsubsection{Liquid crystalline shells}

Colloids with liquid crystal droplets can be produced with relatively simple methods. A recent development made possible by the introduction of somewhat more advanced equipment, a coaxial nested capillary microfluidic system for preparing multiple emulsions with extremely low dispersity [241], is the preparation and study of colloids where the particles are thin shells of liquid crystal rather than droplets. Each shell contains a droplet of an isotropic phase, generally an aqueous surfactant or polymer solution although glycerol or silicon oil can also be used, and these 'composite droplets' are suspended in a continuous phase that can have the same or different composition as the inner droplet phase. Most work has been done with aqueous inner and outer phases since this allows for easy tuning of the director geometry at the shell's inner and outer boundaries. As discussed in the previous subsection, an ionic surfactant yields homeotropic alignment and a polymer like PVA induces planar alignment.

The work in this new field has largely been motivated by a seminal theoretical paper by David Nelson, published in Nano Letters in 2002 [242]. He pointed out that a planar-aligned spherical liquid crystal surface can be extremely interesting as a basis for advanced colloids. The topology of such a system dictates that the liquid crystal director field must have a total defect strength of $s=+2$ over the surface. We are all aware of this situation from the orientational field spanned by the meridians on the earth, which come together into two $s=+1$ topological defects at the north and south pole. The same defect arrangement is possible in a planaraligned spherical liquid crystal surface, but Nelson pointed out that this is not the energy minimizing configuration. By splitting each unit strength defect, or disclination, into two $s=+1 / 2$ defects the energy is reduced, as the energy density of a defect is proportional to the square of its strength. (See Fig. 7 for an overview of the most common nematic topological defects.) Moreover, because the director field in the vicinity of a disclination is distorted, with a consequent elastic energy penalty, the total energy of the spherical system is minimized when the four half-valued defects arrange as to minimize the total distortion of the director field. This happens for a tetrahedral configuration of the disclinations.

The reason that this is particularly interesting is that the defects are high energy points where the liquid crystalline order breaks down, and if a foreign non-liquid crystalline species is added, its molecules may be expected to aggregate at the defects. This would minimize the energy as the foreign species would take up the space where otherwise mesogens must be, putting them in a nonfavorable disordered isotropic state with higher energy than the ordered bulk. If the foreign species are incompatible with liquid crystalline order yet miscible with the liquid crystalline material in its isotropic state, this would further promote migration of that species toward the defects. This type of localization of guest particles or molecules to disordered regions of a liquid crystal host has been used to create soft solids with unusual properties $[181,182]$ and it is thought to be the origin of the dramatic polymer stabilization of liquid crystal blue phases [222], currently strongly explored as a potential basis for next generation high speed displays. Recently it was also used for manipulating and translating polymer molecules within a nematic liquid crystal with localized reduction of orientational order [243].

Nelson's conjecture was that this phenomenon could be used for anchoring ligand molecules to the topological defects in the spherical liquid crystal surface, where the external end of the ligand molecules would have the capacity to bind to other ligands. The 

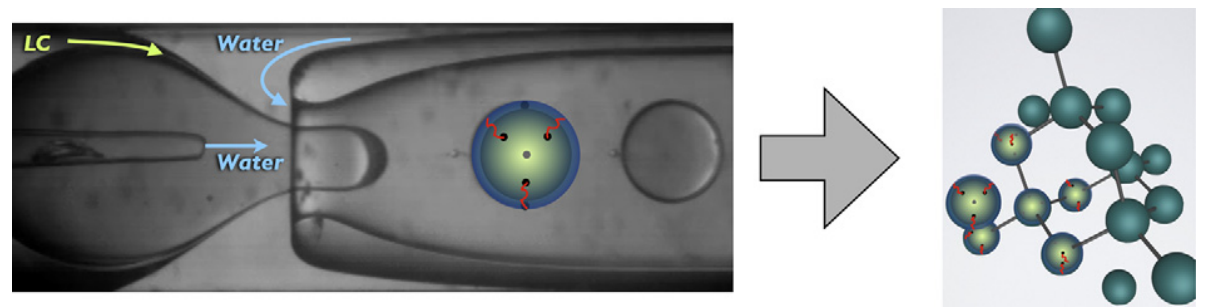

Fig. 17. Production of liquid crystal shells in a nested capillary microfluidic device, together with a cartoon illustrating the postulated self-assembly of ligand-equipped tetravalent liquid crystal shells into a diamond-like colloidal crystal. Microfluidics photograph courtesy of Hsin-Ling Liang.

water soluble ends of the ligand molecules could for instance comprise complementary single DNA strands. Because the disclinations are arranged on a tetrahedron the ligands would give the liquid crystal spheres the possibility to bond in the same way as $s p^{3}$. hybridized carbon does, but on a much larger colloidal, rather than atomic, scale. In this way the shells could self-assemble into colloidal diamond-like crystals, as illustrated schematically in Fig. 17, something which is exceptionally difficult to achieve with regular colloids which typically crystallize in hexagonal closepacked arrangements. A colloidal crystal with a diamond lattice structure is extremely interesting for the photonics community, as this would allow the generation of metamaterials with complete polarization-independent 3D photonic band gap.

One can imagine a few different ways of achieving the required planar-aligned liquid crystal surface but so far the only route that has been pursued is via the microfluidics-produced liquid crystalline shells. The team around David Weitz, Alberto FernandezNieves and Teresa Lopez-Leon were the first to explore this line of research and together they have published a series of beautiful experimental studies of nematic shells [244-247], complemented by theoretical analyses from other groups [248-250]. It turns out that the desired tetrahedral configuration is not quite as easy to achieve in practice as expected, the reason being the extension within the shell of the $s=+1 / 2$ disclination lines. While the energy density is proportional to the square of the defect strength, the actual energy is also proportional to the length of the disclination line. Thus, the liquid crystal typically minimizes the energy by distorting the shell somewhat, rendering it asymmetric with the thinnest point at the top or bottom (in case of imperfect density matching of liquid crystal and inner fluid, which is the usual case), and bringing all four defects close to this regime. Although this increases the director field distortion in the vicinity of the defects, there is a total energy gain due to the reduced extension of the disclination lines within the shell [247].

In an elegant recent study Lopez-Leon and co-workers succeeded in thinning the shell in a controlled way by expanding the internal droplet volume through osmotically driven flow from the continuous phase [245]. Eventually the shell gets so thin that the shell asymmetry is negligible and then the tetrahedral arrangement of four defects can indeed be stabilized. However, this success comes at the cost of expanding the shell diameter quite dramatically, a rather substantial problem considering that the target size is the sub micron range of colloids with attractive optical properties. In fact, the large size of the shells (they are typically in the range of about $100 \mu \mathrm{m}$ ) is one of the two key obstacles on the road toward realizing Nelson's vision. Even without thinning the shell it is very difficult to produce shells that are smaller than some $50-60 \mu \mathrm{m}$ using the microfluidic technique, hence a method to reduce the shell size by about two orders of magnitude is required. This might be possible by producing the shells with a large amount of volatile solvent such as chloroform mixed with the liquid crystal, which could be evaporated after shell production in order to reduce the shell size, but carrying out the evaporation without breaking the shell is a delicate task. It is not unlikely that the microfluidic shell production technique eventually has to be replaced by a different technique that is specifically adapted to the task of producing sub micron shells. The second issue to address is the realization of the required ligand molecules. To date no group has presented any efforts on designing and synthesizing these molecules in practice. While there is probably no fundamental problem preventing their physical realization, it is a challenge that will certainly require some dedicated efforts from skilled synthetic teams, and the chemistry involved is likely to neither be simple nor low cost.

The applied goal of realizing interesting photonic crystal metamaterials using liquid crystal shells is thus still far ahead, with some key challenges remaining to be addressed, but the shells that are currently available are nevertheless extremely attractive as they offer a fantastic playground for fundamental scientific research. For academic research the size range of about $100 \mu \mathrm{m}$ diameter is quite perfect and several groups have published beautiful studies of the textural developments and defect configurations in the shells. The above mentioned studies have given us a good understanding of how the director field and the defects arrange in nematic shells of varying thickness, foremost with planar alignment at both interfaces to the aqueous phases. A study where the alignment at one interface was changed between planar and homeotropic while the other one

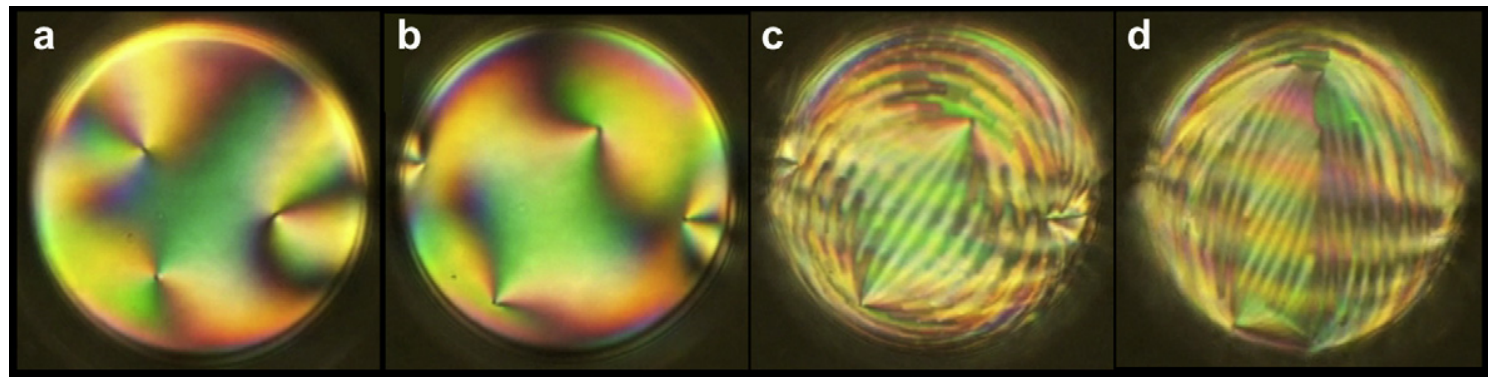

Fig. 18. Transition from the nematic (a, b) to the SmA phase (c, d) in a planar-aligned shell of $8 \mathrm{CB}$. The shell diameter is about $100 \mu \mathrm{m}$. Photographs courtesy of Hsin-Ling Liang, from reference [251]. Copyright (2011) by the American Physical Society. 
was kept planar gave insight into the dynamics as well as the physics prevailing in a nematic shell with hybrid boundary conditions [246].

The first studies on smectic shells, and the transition between nematic and smectic states, were recently presented by Liang et al. [251,252], and Lopez-Leon et al. [253]. The added complexity of 1D positional order here gives rise to new defects and textural features in case of planar-aligned shells, cf. the example in Fig. 18. The frustration of a cross section surface area that diminishes on going from the shell exterior to the interior, and thus a layer number that should get smaller in case of a constant director orientation, leads to buckling instabilities where the director twists from the in- to the outside, with a consequent increasing undulation of the smectic layers [251]. In a recent study Liang et al. also studied the nematic-smectic transition in shells with hybrid boundary conditions, revealing a very rich set of textural features, with beautiful and intriguing transitory textures arising during the transition [254]. A strong dependence on shell size and thickness and on the anchoring strength of the different stabilizers used was also found, providing several handles for tuning the defect configuration in the shell.

The study of liquid crystalline shells is still very much in its infancy, with no studies existing yet on a whole range of liquid crystal phases. The influence of chirality has for instance not at all been investigated so far. The future will be exciting for this field which may hold many surprises of fundamental scientific nature, as well as true progress on the applied track, eventually leading to highly attractive novel metamaterials.

\subsubsection{Liquid crystal-filled electrospun micro-/nanofibers}

A very new development in liquid crystal research that holds considerable application potential is the production of textile fibers functionalized with liquid crystal in the core. With the aim to fabricate a kind of woven electronic paper, Nakata et al. employed a modified melt spinning technique to produce core-sheath polyolefin fibers that contained either cholesteric liquid crystal or an electrophoretic dispersion in the core [255]. In case of short-pitch cholesteric liquid crystal cores the fibers were reflecting in the visible range and the color could be switched off by applying an electric field. This however required a very high driving voltage of $400 \mathrm{~V}$ over the fibers, with outer diameter in the $80-100 \mu \mathrm{m}$ range. While not a traditional textile, the weavability of the produced fibers opens for taking the concept into the direction of smart textiles.

A different approach to producing liquid crystal-core - polymer sheath fibers, with considerably smaller diameter, was recently introduced by Lagerwall and co-workers [256]. Using coaxial electrospinning they could introduce a variety of liquid crystals into the core of the spinning jet, where the sheath fluid was a solution of water soluble polymers like polyvinylpyrrolidone (PVP) or PVA, sometimes with inorganic sol-gel precursor for producing a composite sheath, e.g. with a component of $\mathrm{TiO}_{2}$. Electrospinning is a process for spinning polymer fibers that is rapidly gaining in attention across the international nanoscience and technology and materials science arena. In contrast to conventional techniques the spinning is not driven by high pressure but the polymer fiber is essentially 'pulled' out by a strong electric field from a droplet protruding from the spinneret. The product is a non-woven mat of fibers with diameters typically in the range from some $100 \mathrm{~nm}$ to a few microns. With the simplest collector geometries the mats consist of randomly crisscrossing fibers but it is relatively easy to achieve quite uniform alignment, e.g. by using split electrodes for the collector [257-259]. Part of the beauty of the method is that it requires very simple equipment that easily fits on an ordinary chemistry or physics lab bench and it is very dynamic in the sense that the details of the spinning set-up can easily and quickly be varied, allowing for much experimentation and rapid testing of new ideas. We do not go into the details behind the electrospinning process here but refer the interested reader to one of several excellent current review articles on the topic [260-265] as well as a book chapter specifically devoted to liquid crystal electrospinning [266].

The coaxial electrospinning technique is particularly useful for liquid crystal research and applications as it allows essentially any fluid to be spun in the fiber core, since the strongly non-Newtonian polymer sheath solution stabilizes the liquid jet and prevents the Rayleigh instability to break it up into droplets. In principle, any liquid crystal phase with viscosity low enough to be pumped to the spinneret can thus be incorporated inside electrospun fibers. Lagerwall and co-workers demonstrated this so far on regular nonchiral nematics [256], non-chiral smectic-A [267] as well as shortpitch chiral nematics [268]. In particular the latter is interesting from an applicational point of view, since the selective reflection of the liquid crystal is visible through the sheath for sufficiently thick fibers, thus giving the whole mat the color of the liquid crystal, easily visible by the naked eye. On the other hand, if the mat is heated above the clearing point of the liquid crystal, this turns into an isotropic liquid within the fiber core, removing the color and leaving the mat with a white appearance from light scattering off the polymer sheath, cf. Fig. 19. This simple experiment provided an important demonstration of the application potential of the produced liquid crystal-functionalized mats in sensing devices. The mat was a very basic non-woven textile based temperature sensor, but the same concept can be developed for instance into gas sensors [269-272], where the prime advantages would be low cost and simple production, rapid and sensitive response due to very large surface-to-volume ratio, direct optical read-out by the naked eye by untrained users, full autonomy and independence of power supply, and ease of integration into clothing.

While the coaxial elecrospinning approach is very efficient and provides good control, one can actually achieve the liquid crystal core-polymer sheath fiber mats using conventional electrospinning as well. Buyuktanir et al. mixed standard nematic $5 C B$ with polylactic acid using a mixture of chloroform and acetone as common solvent and spun this using single-fluid electrospinning [273]. As the solvent evaporated the liquid crystal and polymer phase separated, luckily with the liquid crystal primarily in the core, thus contained by the polymer. The simplicity of this approach is very attractive but the degree of control does not quite reach the levels
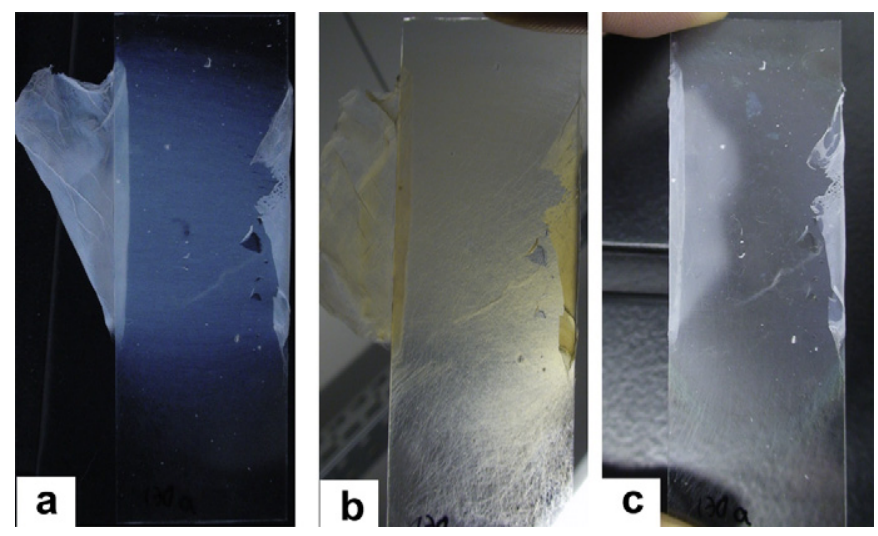

Fig. 19. An electrospun non-woven mat of polyvinylpyrrolidone fibers containing a short-pitch cholesteric liquid crystal mixture in the core. (a) Reflection at room temperature. (b) Transmission at room temperature, showing the complementary redbrown color to the reflected blue color. (c) Reflection after heating above the clearing point. Only the white scattering from the polymer sheath is visible, the color having disappeared with the disappearance of the ordered structure at the cholesteric-isotropic transition. Photographs courtesy of Eva Enz, from reference [268]. Reproduced by permission of The Royal Society of Chemistry. (For interpretation of the references to color in this figure legend, the reader is referred to the web version of this article.) 
of the coaxial electrospinning technique, hence its slightly higher degre of complexity can in many cases be clearly worthwhile.

In terms of the produced fibers the distinguishing feature of electrospinning compared to conventional electrospinning is primarily the very thin diameter. While it is challenging to reach fiber diameters below several tens of microns with conventional spinning methods, due to the dye swell as the polymer solution or melt exits the spinneret, electrospinning frequently yields fibers in the range of tens of nanometers up to a few microns. This gives the produced mats very high surface-to-volume ratio, making them very attractive for instance for sensing applications. It may also be advantageous in terms of bringing down the voltages required for switching the liquid crystal, although the stronger surface anchoring upon reduced inner diameter may raise the threshold voltage. The electrodes required for applying an electric field could be realized in different ways. The whole fiber mat may be laminated between conductive sheets, realized for instance by coating regular textile fibers with carbon nanotubes [274,275], or one could add a third capillary tube to the electrospinning set-up to allow for tripple fluid coaxial spinning. In this way fibers consisting of an intermediate phase between a sheath and a central core material have been realized [276] and if conductive particles such as carbon nanotubes are added to the inner and outer polymer solutions, at a concentration above the percolation threshold, a coaxial electrode geometry would be realized directly in each fiber.

The critical challenge for electrospinning if the target is smart textiles, or wearable technology as it is also called, is the difficulty in producing continuous yarns of the fibers. Electrospinning generally produces non-woven mats whereas attempts to pick up the fibers on a spool, individually or spun into a yarn, generally fail. This makes it impossible to weave or knit fabrics using fibers produced by electrospinning and the prospects for bringing the liquid crystal core fibers to true textile garments are thereby seriously limited. What is sometimes referred to as 'pseudo-woven' mats can be realized by special techniques providing particularly good control of alignment, such that fibers can be spun in subsequent layers of parallel fibers, where the fiber direction of one layer is perpendicular to that of its neighbor layers [277-279]. This may yield mats with better mechanical properties than random nonwovens, although they would have difficulties to compete with woven or knitted textiles.

One approach to incorporate an electrospun non-woven into a textile fabric is to laminate it between sheets of fabric woven from traditional textile fibers, an approach that has been used for incorporating protective filters made out of electrospun fiber mats into garments [280]. This may be a way forward also for the fiber mats functionalized by liquid crystal. Alternatively one might see the liquid crystal electrospinning activity primarily as a useful exploratory phase technique, its versatility being of great value as long as the questions to be answered deal with the spinnability of certain phases, their response to the strong cylindrical encapsulation within the fibers (quite strong effects on liquid crystal phase sequence were found in some of the mats studied so far [256,268]), the response to changes in the environmental conditions or to external fields etc. Once a concept identified as useful should be brought to a stage where true textiles can be produced, this could then be transferred to conventional spinning techniques, a task that should be feasible provided that the very thin diameter of electrospun fibers is not required. For fabrics the fiber diameters are usually much larger, hence this should generally be acceptable.

\subsection{Actuators made from liquid crystal elastomers}

The combination of liquid crystals and polymers is at the core also of another sub field of modern liquid crystal research, that has demonstrated great potential and attracted many players from all over the world. This is the field of liquid crystalline elastomers, produced by weakly cross-linking reactive mesogens present in a thermotropic liquid crystal phase, after ensuring a macroscopically uniform director alignment. The product is a nematic or smectic side- or main-chain polymer rubber with actuating capacity. Due to the long-range order of the liquid crystal the polymer backbone is substantially stretched out in the liquid crystalline state, but if the system is taken to the isotropic state the backbone is free to adopt an entropically favored random coil state instead [281-283]. As a result the whole system shrinks along the initial director orientation, while it expands in the perpendicular dimensions. The transition between the liquid crystalline and isotropic phases can be triggered either by temperature change or by light irradiation, the latter option made possible by the addition of photoisomerizable groups such as azobenzene.

The shape change can be quite dramatic and very fast, and the concept has thus been proposed for use in various types of actuator devices. Films or ribbons of liquid crystal elastomer were used as light driven motors [284] or artificial muscles [285], sheets or disks can respond to light irradiation by bending along any direction, chosen by setting the light polarization [286], or turned into swimmers that move away from a light source [287], and fibers of liquid crystal elastomer showed 'heavy' weight lifting capacity [288]. When a dye-doped short-pitch chiral nematic was turned into a rubber it could be used as a laser with emission wavelength that could be tuned by stretching the rubber [289].

A crucial requirement for achieving efficient actuation from liquid crystal elastomers is a monodomain sample, i.e. the director field must be uniformly aligned throughout the rubber sample. Several techniques have been developed to meet this requirement [290], e.g. starting with a non-polymerized system of reactive mesogens that can be aligned using conventional liquid crystal alignment layers prior to cross-linking, or applying a two-stage cross-linking process with a moment of uniaxial stretching in between. Recently a few interesting alternative approaches were added to the toolbox for making liquid crystal elastomer actuators, including micro- or nanotemplating [291,292] and inkjet printing [293]. Another highly original recent addition, allowing the production of quite peculiar liquid crystal elastomer objects, was introduced by the Zentel group. They pump droplets of a nematic liquid crystalline phase formed by a reactive mesogenic monomer through a microfludic system at high shear rate, such that the director field within each droplet is well-defined [238,294]. During the flow the droplets - which contain photo initiator and cross-linker in addition to the reactive mesogens - are exposed to high-power ultraviolet radiation that ensures a rapid crosslinking, thus turning the droplets into rubber particles. By heating or cooling past the nematic-isotropic transition of these elastomeric particles their shape can be reversibly changed between spherical and ellipsoidal or between oblate and prolate ellipsoid.

This very recent addition to the toolbox of liquid crystal elastomers may provide several new openings for exciting research as well as applications. Although the particles so far are relatively large, the dispersity is small and the potential to dynamically switch between different shapes is clearly very attractive for advanced colloid science. If the particle size can be shrunk to the range of about a micron we can expect very interesting results in terms of colloid crystals which can be made to change symmetry by means of temperature change or light irradiation. Moreover, it can be very interesting to disperse the liquid crystal rubber particles in a non-polymeric liquid crystal host, allowing the dynamic tuning of by changing the shape of the particle by inducing the liquid crystalisotropic phase transition. 
Another compelling recent development along this track is the combination of the liquid crystal shell concept described above with liquid crystal elastomers, explored in a joint effort of the Zentel and Lagerwall teams [295]. By producing the shells with a reactive monomer nematic, doped with cross-linker and photo initiator, and irradiating them with UV light during flow, the fluid shells can be turned into stable shells of rubber, the shape of which can be changed substantially by taking the system past the nematic-isotropic transition. By poking a small hole in the shell with a micropipette this shell demonstrated rapid pumping capacity and the concept could thus be used for realizing one-piece micro pumps that could find use for instance in microfluidics.

\subsection{Liquid crystals in biology, biotechnology, food science and pharmacology}

It is no coincidence that the membranes of the cells in our bodies are in a lyotropic lamellar liquid crystalline state. It provides just the combination of sturdiness and flexibility that life requires. A solid phase would have been far too stiff, rigid and impermeable, whereas an isotropic liquid could not separate the cell from its surroundings or provide a scaffolding for membrane proteins and internal compartments for the organelles. It is thus perhaps not surprising that liquid crystals play a very important role in biology. What is more surprising is that this role has long been largely neglected and that it is only recently that such issues as phase transitions in lipid membranes and the effect of cholesterol on the lyotropic phase sequence of lipids have become a hot topic.

Furthermore, the connection of biology and liquid crystals is not limited to cell membranes. It has long been known that DNA forms liquid crystal phases [296-298], but recently it was even proposed that this very fact may have had a key role in the earliest development of life in the prebiotic era [299-301]. Likewise, present-day cryo electron microscopy techniques have allowed studies of virus capsids that reveal that the viral DNA can be stored so compactly in the capsid because it forms a highly ordered liquid crystalline phase [302-305]. An interesting observation is that many features found in living organisms are very reminiscent of liquid crystal textures, and can often be modeled using descriptions of liquid crystalline order [306-308].

The interest in liquid crystals in biology and the implications of liquid crystalline order for biological processes is today increasing strongly. We end this brief review of groundbreaking activities in modern liquid crystal science by highlighting a few of the themes in this field. First we will however discuss some related topics, where liquid crystals are used as biodetectors or as smart cargo carriers for delayed drug delivery.

\subsubsection{Biodetection with liquid crystals}

Because a reorientation of the director has such dramatic consequences on the optical properties of a liquid crystal, and because the director alignment at an interface is so sensitive to the chemical and physical environment at the interface, liquid crystals have great potential for use in sensors. An example that has attracted strong attention during the last decades is the use of thermotropic nematics for amplifying biological binding events, a research field that was largely pioneered by the team around Nicholas Abbott at the University of Wisconsin-Madison [309-315]. The key idea is to prepare a nematic phase with a particular alignment, either uniformly planar or homeotropic, at an interface with an aqueous phase. Depending on the director alignment the liquid crystal will exhibit a characteristic texture in polarizing microscopy. The analyte is then added to the aqueous phase and if a biomolecular binding event like antigen-antibody recognition takes place at the interface, the liquid crystal will experience a change in its environment at the interface. As a result the director may reorient, modifying the optical properties of the liquid crystal and a distinct change in the polarizing microscopy texture results. The liquid crystal amplifies the binding event such that it becomes easily visible on a microscopic scale. The concept is most often applied to planar liquid crystal samples but also liquid crystal droplets were successfully used for detecting bacteria and viruses [309]. While most biodetectors require fluorescent tagging or labeling of key molecules, this is unnecessary with the liquid crystal-based detection technique. Thus it is often referred to as label-free biodetection.

Recently other researchers extended this concept to detect the presence of single-stranded (ss) DNA using liquid crystals [316,317]. Price and Schwarz [317] prepared a thermotropic nematic at an interface with an aqueous solution of a cationic surfactant, inducing homeotropic director alignment such that the sample appears dark in polarizing microscopy. If ssDNA (anionic) is added, electrostatic complexation between the oppositely charged DNA and surfactant takes place, changing the liquid crystal alignment and thus the texture. If then the complementary DNA strand is added the two strands form a duplex, breaking up the electrostatically complexed DNA-surfactant structure and thus again changing the nematic director orientation, and again the optical appearance changes. A similar approach was followed by Chen and Yang [316] and they even succeeded in quantitative determination of the DNA concentration based on the liquid crystal realignment.

\subsubsection{Drug/nutrient delivery with liquid crystal cubosomes and hexosomes or liquid crystalline cremes}

By replacing the thermotropic cholesteric liquid crystal in the concept of liquid crystal-containing droplets or colloidal particles discussed in Section 3.3.1 by a suitable lyotropic phase, and by bringing the particle size down to the sub micron range, systems with strong application potential in the field of pharmaceutical sciences and functional foods can be realized [318-322]. The lyotropic phase is formed by biocompatible amphiphiles in water, possibly also with an oil (also biocompatible), and the interest has so far been focused primarily on cubic or hexagonal phases. The liquid crystal phase is contained in a thin shell of amphiphilic block co-polymer. These soft matter nanoparticles, generally prepared via ultrasonication giving diameters in the $100 \mathrm{~nm}$ range, are referred to as cubosomes and hexosomes, respectively, and they can be heavily loaded with drugs for oral delivery. Some examples are shown in Fig. 20.

The interest in these rather advanced vessels for drug delivery has several origins $[323,324]$. First, because the core of the particle contains roughly equal amounts of polar and non-polar regimes, the particles can carry hydrophilic as well as hydrophobic drug cargo, even both at the same time. With simpler classic liposomes (also called vesicles, essentially a shell of a lamellar phase membrane of lipids surrounding an aqueous interior) the cargo can only be hydrophilic. A second important advantage is that the drugs are protected in the particle core from chemical or enzymatic degradation during the passage through the digestive system to the site of release. Directly related to this is the fact that the release rate is substantially slower than for simple liposomes due to the more complex soft solid build up of the core. A conventional liposome releases its target immediately once the lipid membrane has been broken through. The slow release and high capacity for hydrophobic load make these liquid crystalline colloidal particles particularly interesting for cancer treatment, as the cytotoxicity of (frequently hydrophobic) cancer drugs may be enhanced.

What is so far primarily discussed in this field in terms of drug delivery can in general easily be translated to nutrient delivery via smart foods incorporating food-grade liquid crystals. Apart from the advantages already listed, lyotropic liquid crystal phases may 

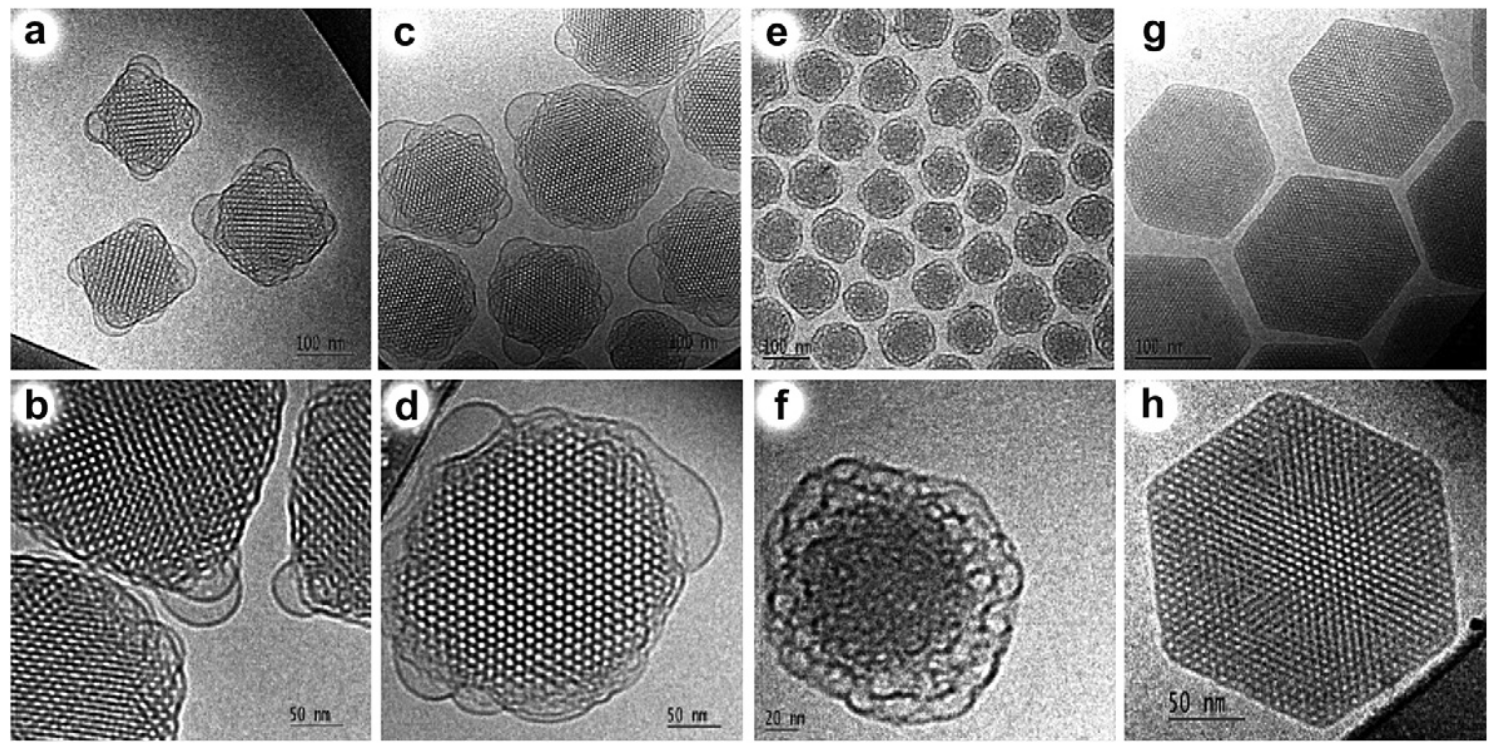

Fig. 20. Cryo-TEM images of soft nanoparticles containing reversed bicontinuous (a-d), sponge (e-f) and reversed hexagonal ( $\mathrm{g}-\mathrm{h}$ ) lyotropic liquid crystal phases. Reprinted with permission from reference [320]. Copyright (2005) American Chemical Society.

also be useful in foods by controlling the texture, viscosity etc. This concept is currently being explored by several major companies active in the technical foods sector.

While this field is fascinating and holds promise, being the basis even for a few companies active in the field, there are some key challenges to address. Primarily they regard robustness and storage stability of the colloidal particles and the scalability issue. When transitioning to a commercial product the production methods employed in a lab are generally not applicable. The cost is another important issue. Encapsulating drugs or nutrients inside colloidal liquid crystalline particles is a rather expensive venture and there must be a substantial gain in efficiency to motivate such a difference. So far, the experimental evidence of added value is not sufficiently convincing. The requirements on biocompatibility/ food-grade materials are also quite restrictive and rule out many options that work well during in-vitro lab experiments for creating stable colloidal particles with desired properties. But perhaps the greatest challenge is the immense complexity of the human body. A myriad of biophysicochemical parameters must be optimized in parallel and the pathway from the mouth to the target site in the body is so complex and involves so many stages with drastically varying environments that it is extremely difficult to find a drug delivery vessel design that withstands the journey.

A slightly less technically advanced way of employing liquid crystals in drug delivery is in topical or other direct application [325,326]. The high viscosity of the liquid crystal phase aids in localization of drugs e.g. at the skin or subcutaneously (under the skin), intramuscularly, or in body cavities such as the mouth. Moreover, transitions between different liquid crystalline phases induced by temperature or concentration change allow dynamic tuning of properties like consistency, shape stability etc. to the requirements of each stage. For example, skin cream can be in a cubic/hexagonal phase at room temperature but melt to lamellar by heating from rubbing, allowing quick and simple administration but reduced stickiness and smeariness afterward as the higher ordered phase is again formed upon cooling. This strategy holds importance also in cosmetics. Another smart example is where a low-viscous solution containing almost no water turns into a viscous liquid crystalline phase when mixed with water at the target location, which in this case would typically be the mouth. In this way the drug can be handled and applied very easily, yet it stays in place once administered.

\subsubsection{Lipidomics and the liquid crystal phases of cell membranes}

As mentioned above the $\mathrm{L}_{\alpha}$ lamellar liquid crystalline phase is the natural state of our cell membranes. In recent years it has become increasingly clear, however, that one actually needs to distinguish two subclasses of this phase. To this end the concepts liquid-ordered $\left(l_{0}\right)$ and liquid-disordered $\left(l_{d}\right)$ states were recently introduced [327-331]. The term "liquid" here refers to the lack of long-range positional order (and thus lack of a lattice) of the lipids within the bilayer plane and a high speed of lateral diffusion, in contrast to the gel phases like $\mathrm{L}_{\beta}^{\prime}$ which in this terminology are considered as "solid" (they do have positional order within the membrane and lateral diffusion is very slow).

The additions "ordered" or "disordered" refer to the state of the acyl chains. The classic $\mathrm{L}_{\alpha}$ picture fits the $l_{d}$ phase best because there are many gauche conformers among the chains and they are very flexible and dynamic. The chains are considered molten, in a random conformation. In the end of the 1980's it was realized, however, that a state can develop where the chains are mainly (albeit not solely) in all-trans conformation, yet without developing the in-plane positional order of the gel phases. Such a state can be described as "liquid" as well as "ordered" and it is thus the $l_{0}$ state. Its appearance is promoted by the presence of cholesterol, the stiff and largely hydrophobic structure of which prefers to be surrounded by ordered alkyl chains and thus enhances the chain ordering. At the same time its bulky shape ruins long-range inplane positional order of the lipids and it thus promotes a "liquid" state over a "gel" state. The consequent frustration is thought to be the origin of the $l_{o}$ phase.

It is not clear if the $l_{0}$ phase can exist macroscopically. It is typically found as microscopic domains suspended in a sea of $l_{d}$ phase in model membranes. The phases thus coexist laterally as a result of phase separation. This situation has given the $l_{0}$ domains the name 'lipid rafts'. A deeper analysis has revealed them as dynamic fluctuating nanoscale regimes enriched in sphingolipids, cholesterol and transmembrane proteins. The surrounding $l_{d}$ 'sea' is instead enriched in ordinary glycerophospholipids. One cannot draw the conclusion that the $l_{0}$ and $l_{d}$ states necessarily occur in live 
cell membranes, since living systems are far from equilibrium. Nevertheless, the lipid raft paradigm with local increases in sphingolipid and cholesterol content has now been generally accepted, also for membranes of living cells. The tendency of this lipid composition to promote increased chain order certainly plays a role in the process, most likely in providing connectivity, which is then further modulated by the specificity of the membrane proteins as well as their antibodies [330].

The investigation of lipid rafts and $l_{o}-l_{d}$ phase coexistence, and the consequences for cell signaling and trafficking, is a very active research area that forms an important component of the emerging field of 'lipidomics'. We can expect very interesting results in the future and as the (still relatively new) liquid-ordered phase becomes better understood this will certainly be a good subject for study from a classic liquid crystal physics point of view. At present, the research is conducted primarily following a biophysical chemistry approach, hence the input from researchers with strong liquid crystal background may provide new and interesting interdisciplinary lines of research. Encouraging examples are given by the work of the Hirst group, USA [331-333], focusing strongly on photo-induced phase separation effects in lipid membranes and tubules, and of the Lee group, Korea, who use surface topography to influence and even pattern lipid rafts [334].

\section{Conclusions and outlook}

We hope that this brief review of the exciting activities in the broad field of liquid crystal research detached from display applications will have inspired the reader as much as the authors are inspired by the research discussed. Several new routes have been initiated during the last few years and some may develop into highly successful research fields and possibly applications, whereas others may turn out not to be so prolific. In most of the fields discussed we are still at a relatively early explorative stage, and we have to wait a few years to assess the potential of each theme. In any case, this is a very exciting development, outlining a bright and rich future for the open-minded liquid crystal scientist or other soft matter nano-/microtechnology researcher with an interest in liquid crystals.

\section{Acknowledgments}

The authors gratefully acknowledge financial support from the National Research Foundation of Korea (NRF), the Advanced Institutes of Convergence Technology (AICT), Korea, and Seoul National University, Korea.

\section{References}

[1] T.J. Sluckin, D.A. Dunmur, H. Stegemeyer, Crystals That Flow: Classic Papers from the History of Liquid Crystals, Taylor and Francis, London, 2004.

[2] R. Stannarius, More than display fillings, Nat. Mater 8 (8) (2009) 617-618.

[3] H.K. Bisoyi, S. Kumar, Liquid-crystal nanoscience: an emerging avenue of soft self-assembly, Chem. Soc. Rev 40 (1) (2011) 306-319.

[4] J.W. Goodby, I. Saez, S. Cowling, V. Gortz, M. Draper, A. Hall, S. Sia, G. Cosquer, S. Lee, E. Raynes, Transmission and amplification of information and properties in nanostructured liquid crystals, Angew. Chem. Int. Ed 47 (15) (2008) $2754-2787$.

[5] T. Kato, Y. Hirai, S. Nakaso, M. Moriyama, Liquid-crystalline physical gels, Chem. Soc. Rev 36 (12) (2007) 1857-1867.

[6] M. Marcos, R. Martin-Rapun, A. Omenat, J. Serrano, Highly congested liquid crystal structures: dendrimers, dendrons, dendronized and hyperbranched polymers, Chem. Soc. Rev 36 (12) (2007) 1889-1901.

[7] S. Sergeyev, W. Pisula, Y. Geerts, Discotic liquid crystals: a new generation of organic semiconductors, Chem. Soc. Rev 36 (12) (2007) 1902-1929.

[8] C. Tschierske, Liquid crystal engineering - new complex mesophase structures and their relations to polymer morphologies, nanoscale patterning and crystal engineering, Chem. Soc. Rev 36 (12) (2007) 1930-1970.

[9] J.W. Goodby, V. Gortz, S. Cowling, G. Mackenzie, P. Martin, D. Plusquellec, T. Benvegnu, P. Boullanger, D. Lafont, Y. Queneau, S. Chambert, J. Fitremann,
Thermotropic liquid crystalline glycolipids, Chem. Soc. Rev 36 (12) (2007) 1971-2032.

[10] R. Lemieux, Molecular recognition in chiral smectic liquid crystals: the effect of core interactions and chirality transfer on polar order, Chem. Soc. Rev 36 (12) (2007) 2033-2045.

[11] C. Imrie, P. Henderson, Liquid crystal dimers and higher oligomers: between monomers and polymers, Chem. Soc. Rev 36 (12) (2007) 2096-2124.

[12] T. Kato, N. Mizoshita, K. Kishimoto, Functional liquid-crystalline assemblies: self-organized soft materials, Angew. Chem. Int. Ed 45 (1) (2006) 38-68.

[13] J.P.F. Lagerwall, Three facets of modern liquid crystal science, Habilitation thesis, Martin-Luther-Universität Halle-Wittenberg.

[14] E. Barry, Z. Dogic, Entropy driven self-assembly of nonamphiphilic colloidal membranes, Proc. Natl. Acad. Sci. U S A 107 (23) (2010) 10348-10353.

[15] M.C. Mourad, J.E. Wijnhoven, D.D. Van't Zand, D. van der Beek, H.N.W. Lekkerkerker, Gelation versus liquid crystal phase transitions in suspensions of plate-like particles, Philos. Transact. A Math. Phys. Eng. Sci 364 (1847) (2006) 2807-2816.

[16] G. Ao, D. Nepal, M. Aono, V. Davis, Cholesteric and nematic liquid crystalline phase behavior of double-stranded DNA stabilized single-walled carbon nanotube dispersions, ACS Nano 5 (2) (2011) 1450-1458.

[17] L. Lu, W. Chen, Large-scale aligned carbon nanotubes from their purified, highly concentrated suspension, ACS Nano 4 (2) (2010) 1042-1048.

[18] S.J. Zhang, I.A. Kinloch, A.H. Windle, Mesogenicity drives fractionation in lyotropic aqueous suspensions of multiwall carbon nanotubes, Nano. Lett 6 (3) (2006) 568-572.

[19] S.H. Aboutalebi, M.M. Gudarzi, Q.B. Zheng, J.-K. Kim, Spontaneous formation of liquid crystals in ultralarge graphene oxide dispersions, Adv. Funct. Mater 21 (15) (2011) 2978-2988.

[20] J.E. Kim, T.H. Han, S.H. Lee, J.Y. Kim, C.W. Ahn, J.M. Yun, S.O. Kim, Graphene oxide liquid crystals, Angew. Chem. Int. Ed 50 (13) (2011) 3043-3047.

[21] F. Guo, F. Kim, T.H. Han, Vivek B. Shenoy, J. Huang, H. Hurt Robert, Hydrationresponsive folding and unfolding in graphene oxide liquid crystal phases, ACS Nano 5 (10) (2011) 8019-8025.

[22] J.P.F. Lagerwall, F. Giesselmann, Current topics in smectic liquid crystal research, Chem. Phys. Chem 7 (1) (2006) 20-45.

[23] P.-G. de Gennes, J. Prost, The Physics of Liquid Crystals, Clarendon Press, Oxford, UK, 1993.

[24] M. O'Neill, S. Kelly, Ordered materials for organic electronics and photonics, Adv. Mater 23 (5) (2011) 566-584.

[25] W. Pisula, M. Zorn, J.Y. Chang, K. Muellen, R. Zentel, Liquid crystalline ordering and charge transport in semiconducting materials, Macromol. Rapid Commun 30 (14) (2009) 1179-1202.

[26] S. Laschat, A. Baro, N. Steinke, F. Giesselmann, C. Hägele, G. Scalia, R. Judele, E. Kapatsina, S. Sauer, A. Schreivogel, M. Tosoni, Discotic liquid crystals: from tailor-made synthesis to plastic electronics, Angew. Chem. Int. Ed 46 (26) (2007) 4832-4887.

[27] M. O'Neill, S. Kelly, Liquid crystals for charge transport, luminescence, and photonics, Adv. Mater 15 (14) (2003) 1135-1146.

[28] J. Harden, M. Chambers, R. Verduzco, P. Luchette, J.T. Gleeson, S. Sprunt, A. Jakli, Giant flexoelectricity in bent-core nematic liquid crystal elastomers, Appl. Phys. Lett 96 (10) (2010) 102907.

[29] A. Jakli, Electro-mechanical effects in liquid crystals, Liq. Cryst 37 (6-7) (2010) 825-837

[30] M. Chambers, R. Verduzco, James T. Gleeson, S. Sprunt, A. Jakli, Calamitic liquid-crystalline elastomers swollen in bent-core liquid-crystal solvents, Adv. Mater 21 (16) (2009) 1622-1626.

[31] M. Chambers, R. Verduzco, J.T. Gleeson, S. Sprunt, A. Jakli, Flexoelectricity of a calamitic liquid crystal elastomer swollen with a bent-core liquid crystal, J. Mater. Chem 19 (42) (2009) 7909-7913.

[32] L. Schmidt-Mende, A. Fechtenkotter, K. Mullen, E. Moons, R. Friend, J. MacKenzie, Self-organized discotic liquid crystals for high-efficiency organic photovoltaics, Science 293 (5532) (2001) 1119-1122.

[33] H. Iino, J. Hanna, Availability of liquid crystallinity in solution processing for polycrystalline thin films, Adv. Mater 23 (15) (2011) 1748-1751.

[34] E. Garcia-Frutos, U. Pandey, R. Termine, A. Omenat, J. Barbera, J. Serrano, A. Golemme, B. Gomez-Lor, High charge mobility in discotic liquidcrystalline triindoles: Just a core business? Angew. Chem. Int. Ed 50 (32) (2011) 7399-7402.

[35] O. Thiebaut, H. Bock, E. Grelet, Face-on oriented bilayer of two discotic columnar liquid crystals for organic donor-acceptor heterojunction, J. Am. Chem. Soc 132 (20) (2010) 6886-6887.

[36] P. Morales, J. Lagerwall, P. Vacca, S. Laschat, G. Scalia, Self-assembled ordered structures in thin films of HAT5 discotic liquid crystal, Beilstein J. Org. Chem 6 (51) (2010). doi:10.3762/bjoc.6.51

[37] Y. Wan, D. Zhao, On the controllable soft-templating approach to mesoporous silicates, Chem. Rev 107 (7) (2007) 2821-2860.

[38] Evan S. Hatakeyama, Christopher J. Gabriel, Brian R. Wiesenauer, Jenny L. Lohr, M. Zhou, D. Noble, D. Richard, Douglas L. Gin, Water filtration performance of a lyotropic liquid crystal polymer membrane with uniform, sub-1-nm pores, J. Membr. Sci 366 (1-2) (2011) 62-72.

[39] L. Sievens-Figueroa, C. Guymon, Cross-linking of reactive lyotropic liquid crystals for nanostructure retention, Chem. Mater 21 (6) (2009) 1060-1068.

[40] D. Gin, J. Bara, R. Noble, B. Elliott, Polymerized lyotropic liquid crystal assemblies for membrane applications, Macromol. Rapid Commun 29 (5) (2008) 367-389. 
[41] J. Clapper, S. Iverson, C. Guymon, Nanostructured biodegradable polymer networks using lyotropic liquid crystalline templates, Biomacromolecules 8 (7) (2007) 2104-2111.

[42] C. Mock-knoblauch, O.S. Enger, U.D. Schalkowsky, Novel polymerisable liquid crystalline acrylates for the manufacturing of ultrathin optical films, SID Dig (2006) 1673-1676.

[43] H. Körner, A. Shiota, T. Bunning, C. Ober, Orientation-on-demand thin films: curing of liquid crystalline networks in ac electric fields, Science 272 (5259) (1996) 252-255.

[44] H. Hentze, E. Kaler, Polymerization of and within self-organized media, Curr. Opin. Colloid Interface Sci 8 (2) (2003) 164-178.

[45] J. Hulvat, S. Stupp, Liquid-crystal templating of conducting polymers, Angew. Chem 42 (7) (2003) 778-781.

[46] M. Goh, S. Matsushita, K. Akagi, From helical polyacetylene to helical graphite: synthesis in the chiral nematic liquid crystal field and morphologyretaining carbonisation, Chem. Soc. Rev 39 (7) (2010) 2466-2476.

[47] Y. Park, Magneto resistance of polyacetylene nanofibers, Chem. Soc. Rev 39 (7) (2010) 2428-2438.

[48] R. Kieffer, M. Prehm, K. Pelz, U. Baumeister, F. Liu, H. Hahn, H. Lang, G. Ungar C. Tschierske, Siloxanes and carbosilanes as new building blocks for $t$-shaped bolaamphiphilic LC molecules, Soft Matter 5 (6) (2009) 1214-1227.

[49] X. Cheng, X. Dong, G. Wei, M. Prehm, C. Tschierske, Liquid-crystalline triangle honeycomb formed by a dithiophene-based $\mathrm{X}$-shaped bolaamphiphile, Angew. Chem. Int. Ed 48 (43) (2009) 8014-8017.

[50] X. Cheng, X. Dong, R. Huang, X. Zeng, G. Ungar, M. Prehm, C. Tschierske, Polygonal cylinder phases of 3-alkyl-2,5-diphenylthiophene-based bolaamphiphiles: changing symmetry by retaining net topology, Chem. Mater 20 (14) (2008) 4729-4738.

[51] F. Liu, B. Chen, B. Glettner, M. Prehm, M. Das, U. Baumeister, X. Zeng, G. Ungar, C. Tschierske, The trapezoidal cylinder phase: a new mode of selfassembly in liquid-crystalline soft matter, J. Am. Chem. Soc 130 (30) (2008) 9666-9667.

[52] B. Glettner, F. Liu, X. Zeng, M. Prehm, U. Baumeister, M. Walker, Martin A. Bates, P. Boesecke, G. Ungar, C. Tschierske, Liquid-crystalline kagome, Angew. Chem. Int. Ed 47 (47) (2008) 9063-9066.

[53] B. Glettner, F. Liu, X. Zeng, M. Prehm, U. Baumeister, G. Ungar, C. Tschierske Liquid-crystal engineering with anchor-shaped molecules: honeycombs with hexagonal and trigonal symmetries formed by polyphilic bent-core molecules, Angew. Chem. Int. Ed 47 (32) (2008) 6080-6083.

[54] F. Liu, B. Chen, U. Baumeister, X. Zeng, G. Ungar, C. Tschierske, The triangula cylinder phase: a new mode of self-assembly in liquid-crystalline soft matter, J. Am. Chem. Soc 129 (31) (2007) 9578-9579.

[55] M. Prehm, F. Liu, U. Baumeister, X. Zeng, G. Ungar, C. Tschierske, The gianthexagon cylinder network - a liquid-crystalline organization formed by a tshaped quaternary amphiphile, Angew. Chem. Int. Ed 46 (42) (2007) 7972-7975.

[56] S. Saliba, P. Davidson, M. Imperor-Clerc, C. Mingotaud, L. Kahn Myrtil, J.D. Marty, Facile direct synthesis of zno nanoparticles within lyotropic liquid crystals: towards organized hybrid materials, J. Mater. Chem 21 (45) (2011) 18191-18194.

[57] G. Surendran, F. Ksar, L. Ramos, B. Keita, L. Nadjo, E. Prouzet, P. Beaunier P. Dieudonne, F. Audonnet, H. Remita, Palladium nanoballs synthesized in hexagonal mesophases, J. Phys. Chem. C 112 (29) (2008) 10740-10744.

[58] R. Guo, B. Zhang, Y. Sun, X. Liu, Lyotropic liquid crystals and applications in synthesis of nanostructured materials, Prog. Chem 19 (11) (2007) 1695-1702.

[59] G. Surendran, L. Ramos, B. Pansu, E. Prouzet, P. Beaunier, F. Audonnet, H. Remita, Synthesis of porous platinum nanoballs in soft templates, Chem Mater 19 (21) (2007) 5045-5048.

[60] G. Karanikolos, P. Alexandridis, R. Mallory, A. Petrou, T. Mountziaris, Templated synthesis of ZnSe nanostructures using lyotropic liquid crystals, Nanotechnology 16 (10) (2005) 2372-2380.

[61] T. Dellinger, P. Braun, Lyotropic liquid crystals as nanoreactors for nanoparticle synthesis, Chem. Mater 16 (11) (2004) 2201-2207.

[62] E. Dos Santos, C. Santilli, S. Pulcinelli, E. Prouzet, Zirconia needles synthesized inside hexagonal swollen liquid crystals, Chem. Mater 16 (21) (2004) 4187-4192.

[63] L. Wang, X. Chen, Z. Sun, Y. Chai, Synthesis of gold nanoplates in lecithin lamellar liquid crystals, Can. J. Chem. Eng 85 (5) (2007) 598-601.

[64] P. Vemula, V. Mallia, K. Bizati, G. John, Cholesterol phenoxy hexanoate mesogens: effect of meta substituents on their liquid crystalline behavior and in situ metal nanoparticle synthesis, Chem. Mater 19 (22) (2007) 5203-5206.

[65] J. Wang, W. Wang, P. Sun, Z. Yuan, B. Li, Q. Jin, D. Ding, T. Chen, Hierarchically helical mesostructured silica nanofibers templated by achiral cationic surfactant, J. Mater. Chem 16 (42) (2006) 4117-4122.

[66] L. Wang, X. Chen, J. Zhan, Y. Chai, C. Yang, L. Xu, W. Zhuang, B. Jing, Synthesis of gold nano- and microplates in hexagonal liquid crystals, J. Phys. Chem. B 109 (2005) 3189-3194.

[67] H. Yang, M. Yang, Y. Zhang, G. Chen, In situ synthesis and lubrication of pbs nanoparticles in lamellar liquid crystal, Colloid J 66 (5) (2004) 635-641.

[68] F. Brochard, P.-G. de Gennes, Theory of magnetic suspensions in liquid crystals, Journal de Physique 31 (7) (1970) 691-708 Paris.

[69] M. Lynch, D. Patrick, Organizing carbon nanotubes with liquid crystals, Nano. Lett 2 (11) (2002) 1197-1201.
[70] I. Dierking, G. Scalia, P. Morales, D. Leclere, Aligning and reorienting carbon nanotubes with nematic liquid crystals, Adv. Mater 16 (11) (2004) 865-869.

[71] W. Lee, C. Wang, Y. Shih, Effects of carbon nanosolids on the electro-optical properties of a twisted nematic liquid-crystal host, Appl. Phys. Lett 85 (4) (2004) 513-515.

[72] H. Chen, W. Lee, Electro-optical characteristics of a twisted nematic liquidcrystal cell doped with carbon nanotubes in a dc electric field, Opt. Rev 12 (3) (2005) 223-225.

[73] C. Huang, C. Hu, H. Pan, K. Lo, Electrooptical responses of carbon nanotubedoped liquid crystal devices, Jpn. J. Appl. Phys 44 (11) (2005) 8077-8081.

[74] I.S. Baik, S.Y. Jeon, S.H. Lee, K.A. Park, S.H. Jeong, K.H. An, Y.H. Lee, Electricalfield effect on carbon nanotubes in a twisted nematic liquid crystal cell, Appl. Phys. Lett 87 (26) (2005) 263110.

[75] H. Duran, B. Gazdecki, A. Yamashita, T. Kyu, Effect of carbon nanotubes on phase transitions of nematic liquid crystals, Liq. Cryst 32 (7) (2005) 815-821.

[76] I. Dierking, G. Scalia, P. Morales, Liquid crystal-carbon nanotube dispersions, J. Appl. Phys 97 (2005) 044309.

[77] I. Dierking, S. San, Magnetically steered liquid crystal-nanotube switch, Appl. Phys. Lett 87 (23) (2005) 233507.

[78] J.P.F. Lagerwall, G. Scalia, M. Haluska, U. Dettlaff-Weglikowska, S. Roth F. Giesselmann, Simultaneous alignment and dispersion of carbon nanotubes with lyotropic liquid crystals, Phys. Stat. Sol (b) 243 (13) (2006) 3046-3049.

[79] J.P.F. Lagerwall, G. Scalia, Carbon nanotubes in liquid crystals, J. Mater. Chem 18 (25) (2008) 2890-2898.

[80] M. Rahman, W. Lee, Scientific duo of carbon nanotubes and nematic liquid crystals, J. Phys. D-Appl. Phys 42 (6) (2009) 063001

[81] G. Scalia, Alignment of carbon nanotubes in thermotropic and lyotropic liquid crystals, Chem. Phys. Chem 11 (2) (2010) 333-340.

[82] G. Scalia, M. Haluska, U. Dettlaff-Weglikowska, F. Giesselmann, S. Roth Polarized raman spectroscopy study of SWCNT orientational order in an aligning liquid crystalline matrix, AIP Conf. Proc 786 (2005) 114-117.

[83] J.P.F. Lagerwall, G. Scalia, M. Haluska, U. Dettlaff-Weglikowska, S. Roth, F. Giesselmann, Nanotube alignment using lyotropic liquid crystals, Adv. Mater 19 (3) (2007) 359-364.

[84] P. van der Schoot, V. Popa-Nita, S. Kralj, Alignment of carbon nanotubes in nematic liquid crystals, J. Phys. Chem. B 112 (15) (2008) 4512-4518.

[85] V. Popa-Nita, S. Kralj, Liquid crystal-carbon nanotubes mixtures, J. Chem. Phys 132 (2) (2010) 024902

[86] M. Cvetko, M. Ambrozic, S. Kralj, Competition between local disordering an global ordering fields in nematic liquid crystals, Beilstein J. Org. Chem 6 (2) (2010). doi:10.3762/bjoc.6.2.

[87] A. Matsuyama, Theory of binary mixtures of a rodlike polymer and a liquid crystal, J. Chem. Phys 132 (21) (2010) 214902.

[88] V. Weiss, R. Thiruvengadathan, O. Regev, Preparation and characterization of a carbon nanotube-lyotropic liquid crystal composite, Langmuir 22 (3) (2006) 854-856.

[89] W. Jiang, B. Yu, W. Liu, J. Hao, Carbon nanotubes incorporated within lyotropic hexagonal liquid crystal formed in room-temperature ionic liquids, Langmuir 23 (16) (2007) 8549-8553.

[90] G. Scalia, C. von Bühler, C. Hägele, S. Roth, F. Giesselmann, J.P.F. Lagerwall, Spontaneous macroscopic carbon nanotube alignment via colloidal suspension in hexagonal columnar lyotropic liquid crystals, Soft Matter 4 (3) (2008) $570-576$.

[91] S. Schymura, E. Enz, S. Roth, G. Scalia, J.P.F. Lagerwall, Macroscopic-scale carbon nanotube alignment via self-assembly in lyotropic liquid crystals, Synthetic Met 159 (21-22) (2009) 2177-2179.

[92] S. Schymura, S. Dölle, J. Yamamoto, J. Lagerwall, Filament formation in carbon nanotube-doped lyotropic liquid crystals, Soft Matter 7 (6) (2011) $2663-2667$.

[93] X. Xin, H. Li, S. Wieczorek, T. Szymborski, E. Kalwarczyk, N. Ziebacz, E. Gorecka, D. Pociecha, R. Holyst, Incorporation of carbon nanotubes into a lyotropic liquid crystal by phase separation in the presence of a hydrophilic polymer, Langmuir 26 (5) (2010) 3562-3568.

[94] H.W. Lee, W. You, S. Barman, S. Hellstrom, C. LeMieux Melburne, J.H. Oh S. Liu, T. Fujiwara, W.M. Wang, B. Chen, Y.W. Jin, J.M. Kim, Z. Bao, Lyotropic liquid-crystalline solutions of high-concentration dispersions of singlewalled carbon nanotubes with conjugated polymers, Small 5 (9) (2009) 1019-1024.

[95] X. Xin, H. Li, E. Kalwarczyk, A. Kelm, M. Fialkowski, E. Gorecka, D. Pociecha R. Holyst, Single-walled carbon nanotube/lyotropic liquid crystal hybrid materials fabricated by a phase separation method in the presence of polyelectrolyte, Langmuir 26 (11) (2010) 8821-8828.

[96] Meagan S. Mauter, M. Elimelech, Chinedum O. Osuji, Nanocomposites of vertically aligned single-walled carbon nanotubes by magnetic alignment and polymerization of a lyotropic precursor, ACS Nano 4 (11) (2010) 6651-6658.

[97] K. Okano, I. Noguchi, T. Yamashita, Anisotropic carbon nanotube film fabricated from a lyotropic liquid-crystalline polymer, Macromolecules 43 (13) (2010) 5496-5499.

[98] F. Tardani, C. La Mesa, Elasticity of dispersions based on carbon nanotubes dissolved in a lyotropic nematic solvent, J. Phys. Chem. C 115 (19) (2011) 9424-9431.

[99] (a) L.N. Lisetski, A.M. Chepikov, S.S. Minenko, N.I. Lebovka, M.S. Soskin, Dispersions of carbon nanotubes in nematic liquid crystals: effects of nanotube geometry, Funct. Mater. 18 (2) (2011) 143-149; 
(b) L.N. Lisetski, N.I. Lebovka, S.V. Naydenov, M.S. Soskin, Dispersions of multiwalled carbon nanotubes in liquid crystals: A physical picture of aggregation, J. Mol. Liq. 164 (2011) 143-147.

[100] J.P.F. Lagerwall, R. Dabrowski, G. Scalia, Antiferroelectric liquid crystals with induced intermediate polar phases and the effects of doping with carbon nanotubes, J. Non-Cryst. Solids 353 (47-51) (2007) 4411-4417

[101] (a) G. Scalia, J.P.F. Lagerwall, S. Schymura, M. Haluska, F. Giesselmann, S. Roth, Carbon nanotubes in liquid crystals as versatile functional materials, Phys. Stat. Sol. (b) 244 (11) (2007) 4212-4217; (b) G. Scalia, J.P.F. Lagerwall, M. Haluska, U. Dettlaff-Weglikowska, F. Giesselmann, S. Roth, Effect of phenyl rings in liquid crystal molecules on SWCNTs studied by Raman spectroscopy, Phys. Stat. Sol. (b) 243 (13) (2006) 3238-3241.

[102] R. Basu, G. Iannacchione, Carbon nanotube dispersed liquid crystal: a nano electromechanical system, Appl. Phys. Lett 93 (18) (2008) 183105.

[103] S. Schymura, M. Kühnast, V. Lutz, S. Jagiella, U. Dettlaff-Weglikowska, S. Roth, F. Giesselmann, C. Tschierske, G. Scalia, J. Lagerwall, Towards efficient dispersion of carbon nanotubes in thermotropic liquid crystals, Adv. Funct. Mater 20 (19) (2010) 3350-3357.

[104] M. Kühnast, C. Tschierske, J. Lagerwall, Tailor-designed polyphilic promotors for stabilizing dispersions of carbon nanotubes in liquid crystals, Chem. Commun 46 (2010) 6989-6991.

[105] Y. Ji, Y. Huang, R. Rungsawang, E. Terentjev, Dispersion and alignment of carbon nanotubes in liquid crystalline polymers and elastomers, Adv. Mater 22 (31) (2010) 3436-3440.

[106] Y. Ji, Y.Y. Huang, Eugene M. Terentjev, Dissolving and aligning carbon nanotubes in thermotropic liquid crystals, Langmuir 27 (21) (2011) $13254-13260$

[107] R. Basu, S. Iannacchione, Germano, Orientational coupling enhancement in a carbon nanotube dispersed liquid crystal, Phys. Rev. E 81 (5) (2010) 051705.

[108] K.P. Sigdel, G.S. Iannacchione, Effect of carbon nanotubes on the isotropic to nematic and the nematic to smectic-a phase transitions in liquid crystal and carbon nanotubes composites, Eur. Phys. J. E 34 (4) (2011) 34.

[109] R. Basu, Krysta A. Boccuzzi, S. Ferjani, C. Rosenblatt, Carbon nanotubeinduced chirality in an achiral liquid crystal, Appl. Phys. Lett 97 (12) (2010) 121908.

[110] R. Basu, Rolfe G. Petschek, C. Rosenblatt, Nematic electroclinic effect in a carbon-nanotube-doped achiral liquid crystal, Phys. Rev. E 83 (4) (2011) 041707.

[111] S. Kumar, H. Bisoyi, Aligned carbon nanotubes in the supramolecular order of discotic liquid crystals, Angew. Chem. Int. Ed 46 (9) (2007) 1501-1503.

[112] H.K. Bisoyi, S. Kumar, Carbon nanotubes in triphenylene and rufigallol-based room temperature monomeric and polymeric discotic liquid crystals, J. Mater. Chem 18 (25) (2008) 3032-3039.

[113] S. Badaire, C. Zakri, M. Maugey, A. Derre, J.N. Barisci, G. Wallace, P. Poulin, Liquid crystals of DNA-stabilized carbon nanotubes, Adv. Mater 17 (13) (2005) 1673-1676.

[114] C. Zakri, P. Poulin, Phase behavior of nanotube suspensions: from attraction induced percolation to liquid crystalline phases, J. Mater. Chem 16 (42) (2006) 4095-4098.

[115] S. Moulton, M. Maugey, P. Poulin, G. Wallace, Liquid crystal behavior of single-walled carbon nanotubes dispersed in biological hyaluronic acid solutions, J. Am. Chem. Soc 129 (30) (2007) 9452-9457.

[116] C. Zamora-Ledezma, C. Blanc, M. Maugey, C. Zakri, P. Poulin, E. Anglaret, Anisotropic thin films of single-wall carbon nanotubes from aligned lyotropic nematic suspensions, Nano. Lett 8 (12) (2008) 4103-4107.

[117] N. Puech, E. Grelet, P. Poulin, C. Blanc, P. van der Schoot, Nematic droplets in aqueous dispersions of carbon nanotubes, Phys. Rev. E 82 (2) (2010) 020702.

[118] W.H. Song, I.A. Kinloch, A.H. Windle, Nematic liquid crystallinity of multiwall carbon nanotubes, Science 302 (5649) (2003) 1363.

[119] W. Song, A. Windle, Isotropic-nematic phase transition of dispersions of multiwall carbon nanotubes, Macromolecules 38 (14) (2005) 6181-6188.

[120] W. Song, A. Windle, Size-dependence and elasticity of liquid-crystalline multiwalled carbon nanotubes, Adv. Mater 20 (16) (2008) 3149-3154.

[121] V. Davis, L. Ericson, A. Parra-vasquez, H. Fan, Y. Wang, V. Prieto, J. Longoria, S. Ramesh, R. Saini, C. Kittrell, W. Billups, W. Adams, R. Hauge, R. Smalley, M. Pasquali, Phase behavior and rheology of SWNTs in superacids, Macromolecules 37 (1) (2004) 154-160.

[122] P. Rai, R. Pinnick, A. Parra-vasquez, V. Davis, H. Schmidt, R. Hauge, R. Smalley, M. Pasquali, Isotropic-nematic phase transition of single-walled carbon nanotubes in strong acids, J. Am. Chem. Soc 128 (2) (2006) 591-595.

[123] A.N.G. Parra-Vasquez, N. Behabtu, Micah J. Green, Cary L. Pint, Colin C. Young, J. Schmidt, E. Kesselman, A. Goyal, Pulickel M. Ajayan, Y. Cohen, Y. Talmon, Robert H. Hauge, M. Pasquali, Spontaneous dissolution of ultralong single- and multiwalled carbon nanotubes, ACS Nano 4 (7) (2010) 3969-3978.

[124] V.A. Davis, Liquid crystalline assembly of nanocylinders, J. Mater. Res 26 (2) (2011) 140-153.

[125] D. Wang, R. Kou, D. Choi, Z. Yang, Z. Nie, J. Li, Laxmikant V. Saraf, D. Hu, J. Zhang, Gordon L. Graff, J. Liu, Michael A. Pope, Ilhan A. Aksay, Ternary selfassembly of ordered metal oxide-graphene nanocomposites for electrochemical energy storage, ACS Nano 4 (3) (2010) 1587-1595.

[126] F. Guo, A. Mukhopadhyay, B. Sheldon, R. Hurt, Vertically aligned graphene layer arrays from chromonic liquid crystal precursors, Adv. Mater 23 (4) (2011) 508-513.
[127] B. Dan, N. Behabtu, A. Martinez, J. Evans, D. Kosynkin, J. Tour, M. Pasquali, I. Smalyukh, Liquid crystals of aqueous, giant graphene oxide flakes, Soft Matter 7 (23) (2011) 11154-11159.

[128] B. Donnio, P. Garcia-Vazquez, J. Gallani, D. Guillon, E. Terazzi, Dendronized ferromagnetic gold nanoparticles self-organized in a thermotropic cubic phase, Adv. Mater 19 (21) (2007) 3534-3539.

[129] M. Draper, Isabel M. Saez, Stephen J. Cowling, P. Gai, B. Heinrich, B. Donnio, D. Guillon, John W. Goodby, Self-assembly and shape morphology of liquidcrystalline gold metamaterials, Adv. Funct. Mater 21 (7) (2011) 1260-1278.

[130] L. Holt, R. Bushby, S. Evans, A. Burgess, G. Seeley, A 10(6)-fold enhancement in the conductivity of a discotic liquid crystal doped with only $1 \%(\mathrm{w} / \mathrm{w})$ gold nanoparticles, J. Appl. Phys 103 (6) (2008) 063712.

[131] Gary M.J. Koenig, Juan J. de Pablo, Nicholas L. Abbott, Characterization of the reversible interaction of pairs of nanoparticles dispersed in nematic liquid crystals, Langmuir 25 (23) (2009) 13318-13321.

[132] P. Kossyrev, A. Yin, S. Cloutier, D. Cardimona, D. Huang, P. Alsing, J. Xu, Electric field tuning of plasmonic response of nanodot array in liquid crystal matrix, Nano. Lett 5 (10) (2005) 1978-1981.

[133] R. Pratibha, W. Park, I.I. Smalyukh, Colloidal gold nanosphere dispersions in smectic liquid crystals and thin nanoparticle-decorated smectic films, J. Appl. Phys 107 (6) (2010) 063511.

[134] Q. Liu, Y. Cui, D. Gardner, X. Li, S. He, I. Smalyukh, Ivan, Self-alignment of plasmonic gold nanorods in reconfigurable anisotropic fluids for tunable bulk metamaterial applications, Nano. Lett 10 (4) (2010) 1347-1353.

[135] H. Park, A. Agarwal, N. Kotov, O. Lavrentovich, Controllable side-by-side and end-to-end assembly of au nanorods by lyotropic chromonic materials, Langmuir 24 (24) (2008) 13833-13837.

[136] H. Qi, J. O'Neil, T. Hegmann, Chirality transfer in nematic liquid crystals doped with (s)-naproxen-functionalized gold nanoclusters: an induced circular dichroism study, J. Mater. Chem 18 (4) (2008) 374-380.

[137] H. Oi, B. Kinkead, T. Hegmann, Unprecedented dual alignment mode and freedericksz transition in planar nematic liquid crystal cells doped with gold nanoclusters, Adv. Funct. Mater 18 (2) (2008) 212-221.

[138] H. Qi, B. Kinkead, Vanessa M. Marx, Huai R. Zhang, T. Hegmann, Miscibility and alignment effects of mixed monolayer cyanobiphenyl liquid-crystalcapped gold nanoparticles in nematic cyanobiphenyl liquid crystal hosts, Chem. Phys. Chem 10 (8) (2009) 1211-1218.

[139] H. Qi, T. Hegmann, Multiple alignment modes for nematic liquid crystals doped with alkylthiol-capped gold nanoparticles, ACS Appl. Mater. Interfaces 1 (8) (2009) 1731-1738.

[140] M. Wojcik, W. Lewandowski, J. Matraszek, J. Mieczkowski, J. Borysiuk, D. Pociecha, E. Gorecka, Liquid-crystalline phases made of gold nanoparticles, Angew. Chem. Int. Ed 48 (28) (2009) 5167-5169.

[141] H. Yoshida, Y. Tanaka, K. Kawamoto, H. Kubo, T. Tsuda, A. Fujii, S. Kuwabata, H. Kikuchi, M. Ozaki, Nanoparticle-stabilized cholesteric blue phases, Appl. Phys. Express 2 (12) (2009) 121501.

[142] X. Zeng, F. Liu, Alan G. Fowler, G. Ungar, L. Cseh, Georg H. Mehl, J.E. Macdonald, 3D ordered gold strings by coating nanoparticles with mesogens, Adv. Mater 21 (17) (2009) 1746.

[143] L. Li, J. Walda, L. Manna, A. Alivisatos, Semiconductor nanorod liquid crystals, Nano. Lett 2 (6) (2002) 557-560.

[144] L. Li, A. Alivisatos, Semiconductor nanorod liquid crystals and their assembly on a substrate, Adv. Mater 15 (5) (2003) 408-411.

[145] L. Shoute, D. Kelley, Spatial organization of GaSe quantum dots: organic/ semiconductor liquid crystals, J. Phys. Chem. C 111 (28) (2007) 10233-10239.

[146] G. Shandryuk, E. Matukhina, R. Vasil'ev, A. Rebrov, G. Bondarenko, A. Merekalov, A. Gas'kov, R. Talroze, Effect of h-bonded liquid crystal polymers on cdse quantum dot alignment within nanocomposite, Macromolecules 41 (6) (2008) 2178-2185.

[147] M. Zorn, S. Meuer, M. Tahir, Y. Khalavka, C. Sönnichsen, W. Tremel, R. Zentel, Liquid crystalline phases from polymer functionalised semiconducting nanorods, J. Mater. Chem 18 (25) (2008) 3050-3058.

[148] T. Zhang, C. Zhong, J. Xu, Cds-nanoparticle-doped liquid crystal displays showing low threshold voltage, Jpn. J. Appl. Phys 48 (5) (2009) 055002.

[149] C. Nobile, L. Carbone, A. Fiore, R. Cingolani, L. Manna, R. Krahne, Selfassembly of highly fluorescent semiconductor nanorods into large scale smectic liquid crystal structures by coffee stain evaporation dynamics, J. Phys.-Condens. Matter 21 (26) (2009) 264013.

[150] B. Kinkead, T. Hegmann, Effects of size, capping agent, and concentration of CdSe and CdTe quantum dots doped into a nematic liquid crystal on the optical and electro-optic properties of the final colloidal liquid crystal mixture, J. Mater. Chem 20 (3) (2010) 448-458.

[151] L. Petti, M. Rippa, A. Fiore, L. Manna, P. Mormile, Dynamic orientational photo-refractive gratings observed in $\mathrm{CdSe} / \mathrm{CdS}$ nanorods doped nematic liquid crystal cells, J. Nonlin. Opt. Phys. Mat 19 (1) (2010) 111-121.

[152] M. Zorn, M.N. Tahir, B. Bergmann, W. Tremel, C. Grigoriadis, G. Floudas, R. Zentel, Orientation and dynamics of $\mathrm{ZnO}$ nanorod liquid crystals in electric fields, Macromol. Rapid Commun 31 (12) (2010) 1101-1107.

[153] J. Mirzaei, M. Urbanski, K. Yu, H.-S. Kitzerow, T. Hegmann, Nanocomposites of a nematic liquid crystal doped with magic-sized CdSe quantum dots, J. Mater. Chem 21 (34) (2011) 12710-12716.

[154] S. Zhang, Candice I. Pelligra, G. Keskar, Pawel W. Majewski, F. Ren, Lisa D. Pfefferle, Chinedum O. Osuji, Liquid crystalline order and magnetocrystalline anisotropy in magnetically doped semiconducting $\mathrm{ZnO}$ nanowires, ACS Nano 5 (10) (2011) 8357-8364. 
[155] M. Ramazanoglu, P. Clegg, R. Birgeneau, C. Garland, M. Neubert, J. Kim, Firstorder isotropic-smectic-a transition in liquid-crystal-aerosil gels, Phys. Rev. E 69 (6) (2004) 061706.

[156] L. Dolgov, O. Yaroshchuk, Electrooptic properties of liquid crystals filled with silica nanoparticles of different sorts, Colloid Polym. Sci 282 (12) (2004) 1403-1408.

[157] J. Payne, E. Thomas, Towards an understanding of nanoparticle-chira nematic liquid crystal co-assembly, Adv. Funct. Mater 17 (15) (2007) 2717-2721.

[158] M. Skarabot, I. Musevic, Direct observation of interaction of nanoparticles in a nematic liquid crystal, Soft Matter 6 (21) (2010) 5476-5481.

[159] E. Venugopal, Suresh K. Bhat, Jijo J. Vallooran, R. Mezzenga, Phase behavior of lipid-based lyotropic liquid crystals in presence of colloidal nanoparticles, Langmuir 27 (16) (2011) 9792-9800.

[160] H. Song, J. Kim, J. Hong, Y. Lee, J. Choi, J. Lee, W. Kim, J. Kim, N. Hur, Magnetic and transparent composites by linking liquid crystals to ferrite nanoparticles through covalent networks, Adv. Funct. Mater 17 (13) (2007) 2070-2076.

[161] P. Kopcansky, N. Tomasovicova, M. Koneracka, V. Zavisova, M. Timko, A. Dzarova, A. Sprincova, N. Eber, K. Fodor-Csorba, T. Toth-Katona, A. Vajda, J. Jadzyn, Structural changes in the 6CHBT liquid crystal doped with spherical, rodlike, and chainlike magnetic particles, Phys. Rev. E 78 (1) (2008) 011702.

[162] C. Lapointe, D. Reich, R. Leheny, Manipulation and organization of ferromagnetic nanowires by patterned nematic liquid crystals, Langmuir 24 (19) (2008) 11175-11181.

[163] G. Cordoyiannis, Lynn K. Kurihara, Luz J. Martinez-Miranda, C. Glorieux J. Thoen, Effects of magnetic nanoparticles with different surface coating on the phase transitions of octylcyanobiphenyl liquid crystal, Phys. Rev. E 79 (1) (2009) 011702

[164] L. Zadoina, B. Lonetti, K. Soulantica, A.F. Mingotaud, M. Respaud, B. Chaudret M. Mauzac, Liquid crystalline magnetic materials, J. Mater. Chem 19 (43) (2009) 8075-8078.

[165] Jijo J. Vallooran, S. Bolisetty, R. Mezzenga, Macroscopic alignment of lyotropic liquid crystals using magnetic nanoparticles, Adv. Mater 23 (34) (2011) 3932-3937.

[166] S. Hernandez-Navarro, P. Tierno, J. Ignes-Mullol, F. Sagues, Breaking the degeneracy of nematic liquid crystals by means of actuated anisometric paramagnetic colloids, Soft Matter 7 (11) (2011) 5109-5112.

[167] O. Buluy, S. Nepijko, V. Reshetnyak, E. Ouskova, V. Zadorozhnii, A. Leonhardt, M. Ritschel, G. Schoenhense, Y. Reznikov, Magnetic sensitivity of a dispersion of aggregated ferromagnetic carbon nanotubes in liquid crystals, Soft Matter 7 (2) (2011) 644-649.

[168] Y. Reznikov, O. Buchnev, O. Tereshchenko, V. Reshetnyak, A. Glushchenko, J. West, Ferroelectric nematic suspension, Appl. Phys. Lett 82 (12) (2003) 1917-1919.

[169] M. Copic, A. Mertelj, O. Buchnev, Y. Reznikov, Coupled director and polarization fluctuations in suspensions of ferroelectric nanoparticles in nematic liquid crystals, Phys. Rev. E 76 (1) (2007) 011702.

[170] L. Lopatina, J. Selinger, Theory of ferroelectric nanoparticles in nematic liquid crystals, Phys. Rev. Lett 102 (19) (2009) 197802.

[171] J. Blach, S. Saitzek, C. Legrand, L. Dupont, J.-F. Henninot, M. Warenghem, BaTiO3 ferroelectric nanoparticles dispersed in 5CB nematic liquid crystal: Synthesis and electro-optical characterization, J. Appl. Phys. 107 (2010) 074102.

[172] O. Kurochkin, H. Atkuri, O. Buchnev, A. Glushchenko, O. Grabar R. Karapinar, V. Reshetnyak, J. West, Y. Reznikov, Nano-colloids of sn2P2S6 in nematic liquid crystal pentyl-cianobiphenile, Condens Matter Phys 13 (3) (2010) 33701

[173] H. Qi, T. Hegmann, Impact of nanoscale particles and carbon nanotubes on current and future generations of liquid crystal displays, J. Mater. Chem 18 (28) (2008) 3288-3294

[174] V. Hsiao, Y. Zheng, B. Juluri, T. Huang, Light-driven plasmonic switches based on au nanodisk arrays and photoresponsive liquid crystals, Adv. Mater 20 (18) (2008) 3528-3532

[175] S. Khatua, P. Manna, W. Chang, A. Tcherniak, E. Friedlander, E.R. Zubarev, S. Link, Plasmonic nanoparticles - liquid crystal composites, J. Phys. Chem. C 114 (2010) 7251-7257.

[176] W. Cai, U. Chettiar, A. Kildishev, V. Shalaev, Optical cloaking with metamaterials, Nat. Photon 1 (4) (2007) 224-227.

[177] V. Shalaev, Optical negative-index metamaterials, Nat. Photon 1 (1) (2007) 41-48.

[178] H. Stark, Director field configurations around a spherical particle in a nematic liquid crystal, Eur. Phys. J. B 10 (2) (1999) 311-321.

[179] P. Cladis, M. Kleman, P. Pieranski, New method for decoration of mesomorphic phase of para methoxybenzilidene para betylaniline, $\mathrm{Cr}$ Acad. Sci. B Phys 273 (6) (1971) 275-279.

[180] P. Poulin, H. Stark, T. Lubensky, D.A. Weitz, Novel colloidal interactions in anisotropic fluids, Science 275 (5307) (1997) 1770-1773.

[181] S.P. Meeker, W.C.K. Poon, J. Crain, E.M. Terentjev, Colloid-liquid-crystal composites: an unusual soft solid, Phys. Rev. E 61 (6) (2000) R6083-R6086.

[182] P. Petrov, E.M. Terentjev, Formation of cellular solid in liquid crystal colloids, Langmuir 17 (10) (2001) 2942-2949.

[183] J. Loudet, P. Hanusse, P. Poulin, Stokes drag on a sphere in a nematic liquid crystal, Science 306 (5701) (2004) 1525.

[184] G. Liao, I. Smalyukh, J. Kelly, O. Lavrentovich, A. Jákli, Electrorotation of colloidal particles in liquid crystals, Phys. Rev. E 72 (3) (2005) 31704.
[185] I. Musevic, M. Skarabot, U. Tkalec, M. Ravnik, S. Zumer, Two-dimensional nematic colloidal crystals self-assembled by topological defects, Science 313 (5789) (2006) 954-958.

[186] L. Foret, A. Onuki, Charged inclusion in nematic liquid crystals, Phys. Rev. E 74 (3) (2006) 031709.

[187] M. Skarabot, U. Tkalec, I. Musevic, Transport and crystallization of colloida particles in a thin nematic cell, Eur. Phys. J. E 24 (1) (2007) 99-107.

[188] O. Pishnyak, S. Tang, J. Kelly, S. Shiyanovskii, O. Lavrentovich, Levitation, lift and bidirectional motion of colloidal particles in an electrically driven nematic liquid crystal, Phys. Rev. Lett 99 (12) (2007) 127802.

[189] M. Skarabot, M. Ravnik, S. Zumer, U. Tkalec, I. Poberaj, D. Babic, N. Osterman, I. Musevic, Two-dimensional dipolar nematic colloidal crystals, Phys. Rev. E 76 (5) (2007) 051406

[190] M. Ravnik, M. Skarabot, S. Zumer, U. Tkalec, I. Poberaj, D. Babic, N. Osterman, I. Musevic, Entangled nematic colloidal dimers and wires, Phys. Rev. Lett 99 (24) (2007) 247801.

[191] I. Musevic, M. Skarabot, Self-assembly of nematic colloids, Soft Matter 4 (2) (2008) 195-199.

[192] M. Skarabot, M. Ravnik, S. Zumer, U. Tkalec, I. Poberaj, D. Babic, N. Osterman, I. Musevic, Interactions of quadrupolar nematic colloids, Phys. Rev. E 77 (3) (2008) 031705

[193] M. Skarabot, M. Ravnik, S. Zumer, U. Tkalec, I. Poberaj, D. Babic, I. Musevic, Hierarchical self-assembly of nematic colloidal superstructures, Phys. Rev. E 77 (6) (2008) 061706

[194] S. Rozhkov, New extended objects in nematics: disclinations, monopoles, pipes, EPL 83 (5) (2008) 56003.

[195] J. Han, Ordered structures of glycerol droplets suspended in nematic liquid crystals, J. Kor. Phys. Soc 54 (2) (2009) 805-808.

[196] V.M. Pergamenshchik, V.A. Uzunova, Colloid-wall interaction in a nematic liquid crystal: the mirror-image method of colloidal nematostatics, Phys. Rev. E 79 (2) (2009) 021704

[197] R. Hung, Francisco, Quadrupolar particles in a nematic liquid crystal: effects of particle size and shape, Phys. Rev. E 79 (2) (2009) 021705.

[198] J.-i. Fukuda, Liquid crystal colloids: a novel composite material based on liquid crystals, J. Phys. Soc. Jpn 78 (4) (2009) 041003.

[199] U. Tkalec, M. Ravnik, S. Zumer, I. Musevic, Vortexlike topological defects in nematic colloids: chiral colloidal dimers and 2D crystals, Phys. Rev. Lett 103 (12) (2009) 127801

[200] K. Tojo, A. Furukawa, T. Araki, A. Onuki, Defect structures in nematic liquid crystals around charged particles, Eur. Phys. J. E 30 (1) (2009) 55-64.

[201] U. Ognysta, A. Nych, V. Nazarenko, M. Skarabot, I. Musevic, Design of 2D binary colloidal crystals in a nematic liquid crystal, Langmuir 25 (20) (2009) $12092-12100$.

[202] M. Conradi, M. Ravnik, M. Bele, M. Zorko, S. Zumer, I. Musevic, Janus nematic colloids, Soft Matter 5 (20) (2009) 3905-3912.

[203] M. Ravnik, Gareth P. Alexander, Julia M. Yeomans, S. Zumer, Mesoscopic modelling of colloids in chiral nematics, Farad. Discuss 144 (2010) 159-169.

[204] M. Bates, G. Skacej, C. Zannoni, Defects and ordering in nematic coatings on uniaxial and biaxial colloids, Soft Matter 6 (3) (2010) 655-663.

[205] U. Tkalec, M. Ravnik, S. Copar, S. Zumer, I. Musevic, Reconfigurable knots and links in chiral nematic colloids, Science 333 (6038) (2011) 62-65.

[206] A. Martinez, Hector C. Mireles, Ivan I. Smalyukh, Large-area optoelastic manipulation of colloidal particles in liquid crystals using photoresponsive molecular surface monolayers, Proc. Natl. Acad. Sci. U S A 108 (52) (2011) 20891-20896.

[207] C. Voltz, R. Stannarius, Self-organization of isotropic droplets in smectic-c free-standing films, Phys. Rev. E 70 (6) (2004) 061702.

[208] C. Bohley, R. Stannarius, Energetics of 2d colloids in free-standing smectic-c films, Eur. Phys. J. E Soft Matter 20 (3) (2006) 299-308.

[209] P. Dolganov, V. Dolganov, Director configuration and self-organization of inclusions in two-dimensional smectic membranes, Phys. Rev. E 73 (4)(2006) 041706

[210] C. Bohley, R. Stannarius, Colloidal inclusions in smectic films with spontaneous bend, Eur. Phys. J. E 23 (1) (2007) 25-30.

[211] C. Bohley, R. Stannarius, Inclusions in free standing smectic liquid crystal films, Soft Matter 4 (4) (2008) 683-702.

[212] P.V. Dolganov, V.K. Dolganov, P. Cluzeau, Behavior of inclusions with different value and orientation of topological dipoles in ferroelectric smectic films, J. Exp. Theor. Phys 109 (1) (2009) 169-175.

[213] M. Humar, M. Skarabot, M. Ravnik, S. Zumer, I. Poberaj, D. Babic, I. Musevic, Electrically tunable diffraction of light from 2D nematic colloidal crystals, Eur. Phys. J. E 27 (1) (2008) 73-79.

[214] J. Pendry, Negative refraction makes a perfect lens, Phys. Rev. Lett 85 (18) (2000) 3966-3969.

[215] J. Pendry, Negative refraction, Contemp Phys 45 (3) (2004) 191-202.

[216] J. Pendry, D. Schurig, D. Smith, Controlling electromagnetic fields, Science 312 (5781) (2006) 1780-1782.

[217] N. Liu, H. Guo, L. Fu, S. Kaiser, H. Schweizer, H. Giessen, Three-dimensional photonic metamaterials at optical frequencies, Nat. Mater 7 (1) (2008) 31-37.

[218] P.P. Crooker, The blue phases - a review of experiments, Liq. Cryst 5 (3) (1989) 751-775

[219] J. Yan, M. Jiao, L. Rao, S.-T. Wu, Direct measurement of electric-field-induced birefringence in a polymer-stabilized blue-phase liquid crystal composite, Opt. Express 18 (11) (2010) 11450-11455.

[220] E. Karatairi, B. Rozic, Z. Kutnjak, V. Tzitzios, G. Nounesis, G. Cordoyiannis, J. Thoen, C. Glorieux, S. Kralj, Nanoparticle-induced widening of the temperature range of liquid-crystalline blue phases, Phys. Rev. E 81 (2010) 041703. 
[221] M. Ravnik, J. Fukuda, J. Yeomans, S. Zumer, Confining blue phase colloids to thin layers, Soft Matter 7 (21) (2011) 10144-10150.

[222] H. Kikuchi, M. Yokota, Y. Hisakado, H. Yang, T. Kajiyama, Polymer-stabilized liquid crystal blue phases, Nat. Mater 1 (1) (2002) 64-68.

[223] D. Yoon, M. Choi, Y. Kim, M. Kim, O. Lavrentovich, H. Jung, Internal structure visualization and lithographic use of periodic toroidal holes in liquid crystals, Nat. Mater 6 (11) (2007) 866-870.

[224] Y. Kim, D. Yoon, H. Jeong, O. Lavrentovich, H. Jung, Smectic liquid crystal defects for self-assembling of building blocks and their lithographic applications, Adv. Funct. Mater 21 (4) (2011) 610-627.

[225] Y. Kim, D. Yoon, H. Jeong, H. Jung, Self-assembled periodic liquid crystal defects array for soft lithographic template, Soft Matter 6 (7) (2010) 1426-1431.

[226] Y.H. Kim, D.K. Yoon, H.S. Jeong, J.H. Kim, E.K. Yoon, H.-T. Jung, Fabrication of a superhydrophobic surface from a smectic liquid-crystal defect array, Adv. Funct. Mater 19 (18) (2009) 3008-3013.

[227] J.H. Kim, Y.H. Kim, H.S. Jeong, E.K. Youn, H.-T. Jung, Highly ordered defect arrays of 8CB (4 '-n-octyl-4-cyano-biphenyl) liquid crystal via templateassisted self-assembly, J. Mater. Chem 21 (45) (2011) 18381-18385.

[228] B. Hamlington, B. Steinhaus, J. Feng, D.R. Link, M. Shelley, A. Shen, Liquid crystal droplet production in a microfluidic device, Liq. Cryst 34 (7) (2007) $861-870$.

[229] J. Loudet, H. Richard, G. Sigaud, P. Poulin, Nonaqueous liquid crystal emulsions, Langmuir 16 (16) (2000) 6724-6730.

[230] M. Humar, I. Musevic, 3D microlasers from self-assembled cholesteric liquidcrystal microdroplets, Opt. Express 18 (26) (2010) 26995-27003.

[231] C. Blanc, M. Kleman, The confinement of smectics with a strong anchoring, Eur. Phys. J. E 4 (2) (2001) 241-251.

[232] H. Coles, S. Morris, Liquid-crystal lasers, Nat. Photon 4 (10) (2010) 676-685.

[233] P. Palffy-Muhoray, W. Cao, M. Moreira, B. Taheri, A. Munoz, Photonics and lasing in liquid crystal materials, Philos. Transact. A Math. Phys. Eng. Sci 364 (1847) (2006) 2747-2761.

[234] M. Ozaki, M. Kasano, T. Kitasho, D. Ganzke, W. Haase, K. Yoshino, Electrotunable liquid-crystal laser, Adv. Mater 15 (12) (2003) 974-977.

[235] W. Cao, A. Munoz, P. Palffy-muhoray, B. Taheri, Lasing in a three-dimensional photonic crystal of the liquid crystal blue phase II, Nat. Mater 1 (2) (2002) 111-113.

[236] K. Landfester, Miniemulsion polymerization and the structure of polymer and hybrid nanoparticles, Angew. Chem. Int. Ed 48 (25) (2009) 4488-4507.

[237] O. Tongcher, R. Sigel, K. Landfester, Liquid crystal nanoparticles prepared as miniemulsions, Langmuir 22 (10) (2006) 4504-4511.

[238] A. Fernandez-Nieves, D. Link, M. Marquez, D. Weitz, Topological changes in bipolar nematic droplets under flow, Phys. Rev. Lett 98 (8) (2007) 087801.

[239] A. Fernandez-Nieves, G. Cristobal, V. Garces-Chavez, G. Spalding, K. Dholakia, D. Weitz, Optically anisotropic colloids of controllable shape, Adv. Mater 17 (6) (2005) 680-684.

[240] R. Won, Liquid crystals tiny tunable 3D lasers, Nat. Photon 5 (3) (2011) 133.

[241] A. Utada, E. Lorenceau, D.R. Link, P.D. Kaplan, H.A. Stone, D.A. Weitz, Monodisperse double emulsions generated from a microcapillary device, Science 308 (5721) (2005) 537-541.

[242] D. Nelson, Toward a tetravalent chemistry of colloids, Nano. Lett 2 (10) (2002) 1125-1129.

[243] S. Samitsu, Y. Takanishi, J. Yamamoto, Molecular manipulator driven by spatial variation of liquid-crystalline order, Nat. Mater 9 (10) (2010) 816-820.

[244] T. Lopez-Leon, A. Fernandez-Nieves, Drops and shells of liquid crystal, Colloid Polym. Sci 289 (4) (2011) 345-359.

[245] T. Lopez-Leon, V. Koning, K.B.S. Devaiah, V. Vitelli, A. Fernandez-Nieves, Frustrated nematic order in spherical geometries, Nat. Phys 7 (2011) 391-394.

[246] T. Lopez-Leon, A. Fernandez-Nieves, Topological transformations in bipolar shells of nematic liquid crystals, Phys. Rev. E 79 (2) (2009) 021707.

[247] A. Fernandez-Nieves, V. Vitelli, A. Utada, D.R. Link, M. Marquez, D.R. Nelson, D.A. Weitz, Novel defect structures in nematic liquid crystal shells, Phys. Rev. Lett 99 (15) (2007) 157801.

[248] X. Xing, Topology and geometry of smectic order on compact curved substrates, J. Stat. Phys 134 (3) (2009) 487-536.

[249] H. Shin, M. Bowick, X. Xing, Topological defects in spherical nematics, Phys. Rev. Lett 101 (3) (2008) 037802.

[250] G. Skacej, C. Zannoni, Controlling surface defect valence in colloids, Phys. Rev. Lett 100 (19) (2008) 197802

[251] H.-L. Liang, S. Schymura, P. Rudquist, J. Lagerwall, Nematic-smectic transition under confinement in liquid crystalline colloidal shells, Phys. Rev. Lett 106 (24) (2011) 247801.

[252] H.-L. Liang, E. Enz, G. Scalia, J. Lagerwall, Liquid crystals in novel geometries prepared by microfluidics and electrospinning, Mol. Cryst. Liq. Cryst 549 (2011) 69-77.

[253] T. Lopez-Leon, A. Fernandez-Nieves, M. Nobili, C. Blanc, Nematic-smectic transition in spherical shells, Phys. Rev. Lett 106 (24) (2011) 247802.

[254] H.-L. Liang, R. Zentel, P. Rudquist, J. Lagerwall, Towards tunable defect arrangements in smectic liquid crystal shells utilizing the nematic-smectic transition in hybrid-aligned geometries, Soft Matter, 2012.

[255] M. Nakata, M. Sato, Y. Matsuo, S. Maeda, S. Hayashi, Hollow fibers containing various display elements: a novel structure for electronic paper, J. SID 14 (8) (2006) 723-727.

[256] J.P.F. Lagerwall, J.T. McCann, E. Formo, G. Scalia, Y. Xia, Coaxial electrospinning of microfibres with liquid crystal in the core, Chem. Commun 42 (2008) 5420-5422.
[257] H. Yan, L. Liu, Z. Zhang, Alignment of electrospun nanofibers using dielectric materials, Appl. Phys. Lett 95 (14) (2009) 143114.

[258] R. Dersch, T. Liu, A. Schaper, A. Greiner, J. Wendorff, Electrospun nanofibers: internal structure and intrinsic orientation, J. Polym. Sci. A 41 (4) (2003) $545-553$.

[259] D. Li, Y. Wang, Y. Xia, Electrospinning of polymeric and ceramic nanofibers as uniaxially aligned arrays, Nano. Lett 3 (8) (2003) 1167-1171.

[260] A. Yarin, Coaxial electrospinning and emulsion electrospinning of core-shell fibers, Polym. Advan Technol 22 (3) (2011) 310-317.

[261] L. Bellan, H. Craighead, Applications of controlled electrospinning systems, Polym. Advan Technol 22 (3) (2011) 304-309.

[262] X. Lu, C. Wang, Y. Wei, One-dimensional composite nanomaterials: synthesis by electrospinning and their applications, Small 5 (21) (2009) 2349-2370.

[263] D. Reneker, A. Yarin, Electrospinning jets and polymer nanofibers, Polymer 49 (10) (2008) 2387-2425

[264] A. Greiner, J. Wendorff, Electrospinning: a fascinating method for the preparation of ultrathin fibres, Angew. Chem. Int. Ed 46 (30) (2007) 5670-5703.

[265] D. Li, Y. Xia, Electrospinning of nanofibers: reinventing the wheel? Adv. Mater 16 (14) (2004) 1151-1170.

[266] J.P.F. Lagerwall, Liquid crystal-functionalized nano- and microfibers produced by electrospinning, in: Q. Li (Ed.), Liquid Crystals Beyond Display Applications, John Wiley \& Sons, 2012.

[267] E. Enz, U. Baumeister, J. Lagerwall, Coaxial electrospinning of liquid crystalcontaining poly(vinyl pyrrolidone) microfibers, Beilstein J. Org. Chem 5 (58) (2009). doi:10.3762/bjoc.5.58.

[268] E. Enz, J. Lagerwall, Electrospun microfibres with temperature sensitive iridescence from encapsulated cholesteric liquid crystal, J. Mater. Chem 20 (33) (2010) 6866-6872.

[269] Y. Han, K. Pacheco, C.W.M. Bastiaansen, D.J. Broer, R.P. Sijbesma, Optical monitoring of gases with cholesteric liquid crystals, J. Am. Chem. Soc 132 (9) (2010) 2961-2967.

[270] A. Mujahid, H. Stathopulos, A. Lieberzeit, Peter, L. Dickert, Franz, Solvent vapour detection with cholesteric liquid crystals-optical and mass-sensitive evaluation of the sensor mechanism, Sensors 10 (5) (2010) 4887-4897.

[271] L. Sutarlie, H. Qin, K.-L. Yang, Polymer stabilized cholesteric liquid crystal arrays for detecting vaporous amines, Analyst 135 (7) (2010) 1691-1696.

[272] D. Winterbottom, R. Narayanaswamy, I. Raimundo, Cholesteric liquid crystals for detection of organic vapours, Sensor. Actuat. B 90 (1-3) (2003) 52-57.

[273] Ebru A. Buyuktanir, Margaret W. Frey, John L. . West, Self-assembled, optically responsive nematic liquid crystal/polymer core-shell fibers: Formation and characterization, Polymer 51 (21) (2010) 4823-4830.

[274] Y. Liu, X. Wang, K. Qi, J. Xin, Functionalization of cotton with carbon nanotubes, J. Mater. Chem 18 (29) (2008) 3454-3460.

[275] B. Shim, W. Chen, C. Doty, C. Xu, N. Kotov, Smart electronic yarns and wearable fabrics for human biomonitoring made by carbon nanotube coating with polyelectrolytes, Nano. Lett 8 (12) (2008) 4151-4157.

[276] H. Chen, N. Wang, J. Di, Y. Zhao, Y. Song, L. Jiang, Nanowire-in-microtube structured core/shell fibers via multifluidic coaxial electrospinning, Langmuir 26 (13) (2010) 11291-11296.

[277] C. Hellmann, J. Belardi, R. Dersch, A. Greiner, J. Wendorff, S. Bahnmueller, High precision deposition electrospinning of nanofibers and nanofiber nonwovens, Polymer 50 (5) (2009) 1197-1205.

[278] L. Carnell, E. Siochi, N. Holloway, R. Stephens, C. Rhim, L. Niklason, R. Clark, Aligned mats from electrospun single fibers, Macromolecules 41 (2008) 5345-5349.

[279] C. Chang, K. Limkrailassiri, L. Lin, Continuous near-field electrospinning for large area deposition of orderly nanofiber patterns, Appl. Phys. Lett 93 (12) (2008) 123111.

[280] S. Lee, S. Obendorf, Developing protective textile materials as barriers to liquid penetration using melt-electrospinning, J. Appl. Polym. Sci 102 (4) (2006) 3430-3437.

[281] M. Warner, E.M. Terentjev, Liquid Crystal Elastomers (International Series of Monographs on Physics), Oxford University Press, USA, 2007.

[282] H. Yang, G. Ye, X. Wang, P. Keller, Micron-sized liquid crystalline elastomer actuators, Soft Matter 7 (3) (2011) 815-823.

[283] C. Ohm, M. Brehmer, R. Zentel, Liquid crystalline elastomers as actuators and sensors, Adv. Mater 22 (31) (2010) 3366-3387.

[284] M. Yamada, M. Kondo, J. Mamiya, Y. Yu, M. Kinoshita, C. Barrett, T. Ikeda, Photomobile polymer materials: towards light-driven plastic motors, Angew. Chem. Int. Ed 47 (27) (2008) 4986-4988.

[285] D. Thomsen, P. Keller, J. Naciri, R. Pink, H. Jeon, D. Shenoy, B. Ratna, Liquid crystal elastomers with mechanical properties of a muscle, Macromolecules 34 (17) (2001) 5868-5875.

[286] Y. Yu, M. Nakano, T. Ikeda, Directed bending of a polymer film by light miniaturizing a simple photomechanical system could expand its range of applications, Nature 425 (6954) (2003) 145.

[287] M. Camacho-Lopez, H. Finkelmann, P. Palffy-Muhoray, M. Shelley, Fast liquidcrystal elastomer swims into the dark, Nat. Mater 3 (5) (2004) 307-310.

[288] C. Ohm, M. Morys, F.R. Forst, L. Braun, A. Eremin, C. Serra, R. Stannarius, R. Zentel, Preparation of actuating fibres of oriented main-chain liquid crystalline elastomers by a wetspinning process, Soft Matter 7 (8) (2011) 3730-3734.

[289] H. Finkelmann, S. Kim, A. Munoz, P. Palffy-Muhoray, B. Taheri, Tunable mirrorless lasing in cholesteric liquid crystalline elastomers, Adv. Mater 13 (14) (2001) 1069-1072. 
[290] M. Li, P. Keller, Artificial muscles based on liquid crystal elastomers, Philos. Transact. A Math. Phys. Eng. Sci 364 (1847) (2006) 2763-2777.

[291] C. Ohm, N. Haberkorn, P. Theato, R. Zentel, Template-based fabrication of nanometer-scaled actuators from liquid-crystalline elastomers, Small 7 (2) (2011) 194-198.

[292] A. Buguin, M. Li, P. Silberzan, B. Ladoux, P. Keller, Micro-actuators: when artificial muscles made of nematic liquid crystal elastomers meet soft lithography, J. Am. Chem. Soc 128 (4) (2006) 1088-1089.

[293] Casper L. van Oosten, Cees W.M. Bastiaansen, Dirk J. Broer, Printed artificial cilia from liquid-crystal network actuators modularly driven by light, Nat. Mater 8 (8) (2009) 677-682.

[294] C. Ohm, E. Fleischmann, I. Kraus, C. Serra, R. Zentel, Control of the Properties of Micrometer Sized Actuators from Liquid Crystalline Elastomers Prepared in a Microfluidic Setup, Adv. Funct. Mater 20 (24) (2010) 4314-4322.

[295] E.-K. Fleischman, H.-L. Liang, J. Lagerwall, R. Zentel, Towards micromete sized core-shell actuators from liquid crystalline elastomers by a continuous flow synthesis, SPIE Photonics West 8279 (2012)

[296] R. Franklin, R. Gosling, Molecular configuration in sodium thymonucleate, Nature 171 (4356) (1953) 740-741.

[297] M. Wilkins, A. Stokes, H. Wilson, Molecular structure of deoxypentose nucleic acids, Nature 171 (4356) (1953) 738-740.

[298] J.E. Lydon, The DNA double helix - the untold story, Liq. Cryst. Today 12 (2) (2003) 1-9.

[299] G. Zanchetta, T. Bellini, M. Nakata, N. Clark, Physical polymerization and liquid crystallization of RNA oligomers, J. Am. Chem. Soc 130 (39) (2008) $12864-12865$.

[300] G. Zanchetta, M. Nakata, M. Buscaglia, T. Bellini, N.A. Clark, Phase separation and liquid crystallization of complementary sequences in mixtures of nanoDNA oligomers, Proc. Natl. Acad. Sci. U S A 105 (4) (2008) 1111-1117.

[301] M. Nakata, G. Zanchetta, B.D. Chapman, C.D. Jones, J.O. Cross, R. Pindak, T. Bellini, N.A. Clark, End-to-end stacking and liquid crystal condensation of 6 to 20 base pair DNA duplexes, Science 318 (5854) (2007) 1276-1279.

[302] A. Leforestier, F. Livolant, The bacteriophage genome undergoes a succession of intracapsid phase transitions upon DNA ejection, J. Mol. Biol 396 (2) (2010) 384-395

[303] A. Leforestier, F. Livolant, Structure of toroidal DNA collapsed inside the phage capsid, Proc. Natl. Acad. Sci. U S A 106 (23) (2009) 9157-9162.

[304] A. Leforestier, A. Berlin, J. Dubochet, K. Richter, N. Blanc, F. Livolant Expression of chirality in columnar hexagonal phases or DNA and nucleosomes, C. R. Chim 11 (3) (2008) 229-244.

[305] A. Leforestier, S. Brasiles, M. de Frutos, E. Raspaud, L. Letellier, P. Tavares, F. Livolant, Bacteriophage t5 DNA ejection under pressure, J. Mol. Biol 384 (3) (2008) 730-739.

[306] Y. Bouligand, Liquid crystals and biological morphogenesis: ancient and new questions, C. R. Chim 11 (3) (2008) 281-296.

[307] Y. Bouligand, Liquid crystals and their stabilized analogues as "templates" of biocomposites, Annales De Chimie-science Des Materiaux 29 (1) (2004) 83-96.

[308] Y. Bouligand, The renewal of ideas about biomineralisations, Comptes Rendus-Palevol 3 (6-7) (2004) 617-628.

[309] S. Sivakumar, Kim L. Wark, Jugal K. Gupta, Nicholas L. Abbott, F. Caruso, Liquid crystal emulsions as the basis of biological sensors for the optical detection of bacteria and viruses, Adv. Funct. Mater 19 (14) (2009) 2260-2265.

[310] J. Gupta, M. Meli, S. Teren, N. Abbott, Elastic energy-driven phase separation of phospholipid monolayers at the nematic liquid-crystal-aqueous interface, Phys. Rev. Lett 1 (4) (2008) 048301.

[311] C. Jang, L. Cheng, C. Olsen, N. Abbott, Anchoring of nematic liquid crystals on viruses with different envelope structures, Nano. Lett 6(5)(2006) 1053-1058.
[312] N. Lockwood, J. Mohr, L. Ji, C. Murphy, S. Palecek, J. De Pablo, N. Abbott, Thermotropic liquid crystals as substrates for imaging the reorganization of matrige by human embryonic stem cells, Adv. Funct. Mater 16 (5) (2006) 618-624.

[313] J. Brake, M. Daschner, Y. Luk, N. Abbott, Biomolecular interactions at phospholipid-decorated surfaces of liquid crystals, Science 302 (5653) (2003) 2094-2097.

[314] S. Kim, N. Abbott, Rubbed films of functionalized bovine serum albumin as substrates for the imaging of protein-receptor interactions using liquid crystals, Adv. Mater 13 (19) (2001) 1445-1449.

[315] V. Gupta, J. Skaife, T. Dubrovsky, N. Abbott, Optical amplification of ligandreceptor binding using liquid crystals, Science 279 (5359) (1998) 2077-2080.

[316] C.-H. Chen, K.-L. Yang, Detection and quantification of DNA adsorbed on solid surfaces by using liquid crystals, Langmuir 26 (3) (2010) 1427-1430.

[317] A. Price, D. Schwartz, DNA hybridization-induced reorientation of liquid crystal anchoring at the nematic liquid crystal/aqueous interface, J. Am. Chem. Soc 130 (26) (2008) 8188-8194.

[318] A. Yaghmur, O. Glatter, Characterization and potential applications of nanostructured aqueous dispersions, Adv. Colloid Interface Sci 147-48 (2009) 333-342.

[319] L. Sagalowicz, M. Leser, H. Watzke, M. Michel, Monoglyceride self-assembly structures as delivery vehicles, Trends. Food Sci. Tech 17 (5) (2006) 204-214.

[320] J. Barauskas, M. Johnsson, F. Tiberg, Self-assembled lipid superstructures: beyond vesicles and liposomes, Nano. Lett 5 (8) (2005) 1615-1619.

[321] R. Mezzenga, P. Schurtenberger, A. Burbidge, M. Michel, Understanding foods as soft materials, Nat. Mater 4 (10) (2005) 729-740.

[322] P. Spicer, Progress in liquid crystalline dispersions: cubosomes, Curr. Opin Colloid Interface Sci 10 (5-6) (2005) 274-279.

[323] O. Farokhzad, R. Langer, Impact of nanotechnology on drug delivery, ACS Nano 3 (1) (2009) 16-20.

[324] M. Malmsten, Soft drug delivery systems, Soft Matter 2 (9) (2006) 760-769.

[325] S. Savic, G. Vuleta, R. Daniels, C. Muller-goymann, Colloidal microstructure of binary systems and model creams stabilized with an alkylpolyglucoside nonionic emulsifier, Colloid Polym. Sci 283 (4) (2005) 439-451.

[326] C. Muller-goymann, Physicochernical characterization of colloidal drug delivery systems such as reverse micelles, vesicles, liquid crystals and nanoparticles for topical administration, Eur. J. Pharm. Biopharm 58 (2) (2004) 343-356.

[327] Ole G. Mouritsen, The liquid-ordered state comes of age, Biochim. Biohys. Acta-Biomembr 1798 (7) (2010) 1286-1288.

[328] O.G. Mouritsen, Life - As a Matter of Fat, Springer, 2004.

[329] K. Simons, M. Gerl, Revitalizing membrane rafts: new tools and insights, Nat Rev. Mol. Cell Bio 11 (10) (2010) 688-699.

[330] D. Lingwood, K. Simons, Lipid rafts as a membrane-organizing principle, Science 327 (5961) (2010) 46-50.

[331] L.S. Hirst, P. Uppamoochikkal, C. Lor, Phase separation and critical phenomena in biomimetic ternary lipid mixtures, Liq. Cryst 38 (11-12) (2011) 1735-1747.

[332] Linda S. Hirst, J. Yuan, The effects of fluorescent probes on model membrane organization: photo-induced lipid sorting and soft structure formation, Liq Cryst $36(6-7)$ (2009) 739-745.

[333] J. Yuan, S. Hira, G. Strouse, L. Hirst, Lipid bilayer discs and banded tubules: photoinduced lipid sorting in ternary mixtures, J. Am. Chem. Soc 130 (6) (2008) 2067-2072.

[334] T. Yoon, C. Jeong, S. Lee, J. Kim, M. Choi, S. Kim, M. Kim, S. Lee, Topographic control of lipid-raft reconstitution in model membranes, Nat. Mater 5 (4) (2006) 281-285. 